\title{
MICROWAVE INTERFEROMETRY DIAGNOSTIC APPLICATIONS FOR MEASUREMENTS OF EXPLOSIVES
}

\author{
A Thesis \\ presented to \\ the Faculty of California Polytechnic State University, \\ San Luis Obispo
}

\author{
In Partial Fulfillment \\ of the Requirements for the Degree \\ Master of Science in Electrical Engineering
}

by

Loren Andrew Kline

September 2017 
(C) 2017

Loren Andrew Kline

ALL RIGHTS RESERVED 
TITLE: Microwave Interferometry Diagnostic Applications for Measurements of Explosives

AUTHOR: Loren Andrew Kline

DATE SUBMITTED: $\quad$ September 2017

\begin{abstract}
COMMITTEE CHAiR: Bruce DeBruhl, Ph.D. Assistant Professor of Computer Science and Software Engineering

COMMITTEE MEMBER: Wayne Pilkington, Ph.D. Associate Professor of Electrical Engineering

COMMITTEE MEMBER: Tina Smilkstein, Ph.D. Associate Professor of Electrical Engineering
\end{abstract}




\section{ABSTRACT}

Microwave Interferometry Diagnostic Applications for Measurements of Explosives

\section{Loren Andrew Kline}

Microwave interferometry (MI) is a Doppler based diagnostic tool used to measure the detonation velocity of explosives, which has applications to explosive safety. The geometry used in existing MI experiments is cylindrical explosives pellets layered in a cylindrical case. It is of interest to Lawrence Livermore National Labs to measure additional geometries that may be overmoded, meaning that the geometries propagate higher-order transverse electromagnetic waves. The goal of my project is to measure and analyze the input reflection from a novel structure and to find a good frequency to use in an experiment using this structure. Two methods of determining a good frequency are applied to the phase of the input reflection. The first method is $R^{2}$, used to measure the linearity of input reflection phase. The second is a zerocrossing method that measures how periodic the input reflection phase is. Frequencies with $\mathrm{R}^{2}$ values higher than 0.995 may be usable for an experiment in the novel structure.

Keywords: Explosives, Microwave Interferometry, Quadrature Analysis, Zero-Crossings, $R^{2}$ Analysis, Shock-to-Detonation Transition, Deflagration-to-Detonation Transition 


\section{ACKNOWLEDGEMENTS}

I thank my small team, including Emer, Ron, Joe, Mark, and Owen, who worked with me to get this final paper finished and learned this topic with me. I thank LLNL for hiring me and giving me employment for the co-op that allowed me to do this work. I thank Bruce for serving as my advisor. I thank my mother and father who pushed me to succeed and who without, I certainly would not have made it through college. And most importantly, I would like to thank God for giving to me everything I know and everything I have. 


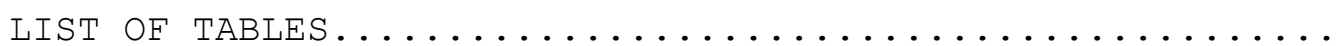

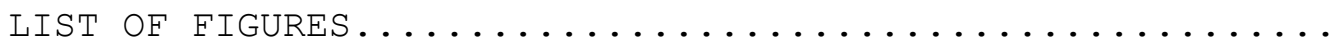

viii

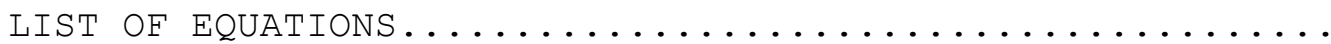

xii

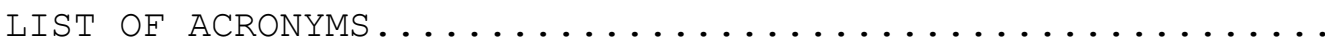

xiii CHAPTERS

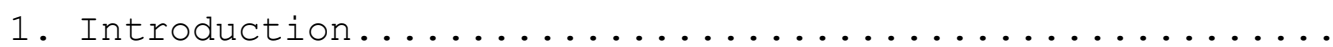

01

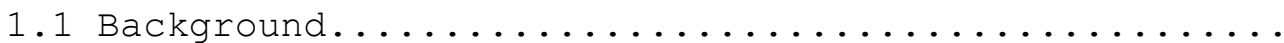

01

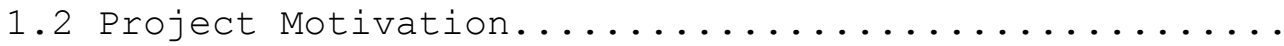

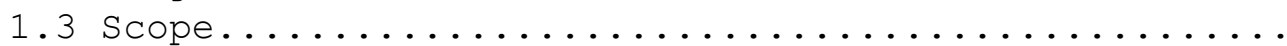

01

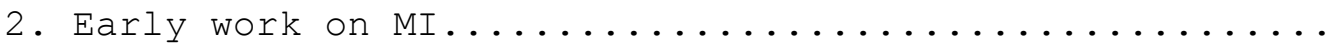

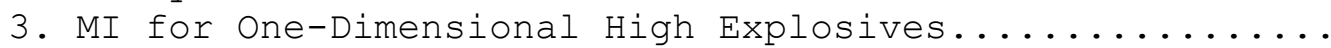

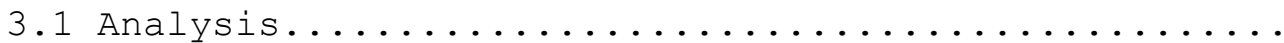

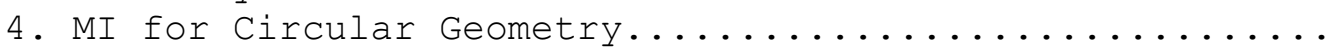

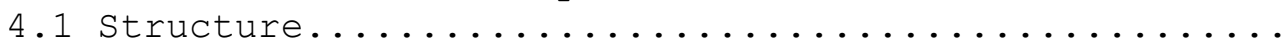

4.2 Matching Improvements by Brewster Angle..........

4.3 Matching Improvements by Material Matching........

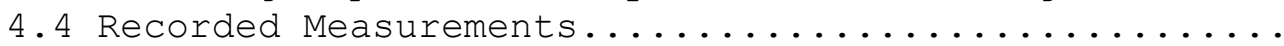

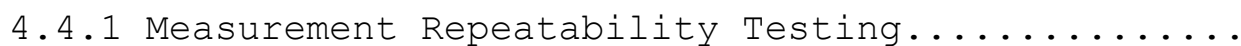

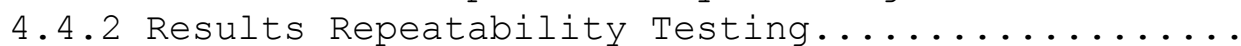

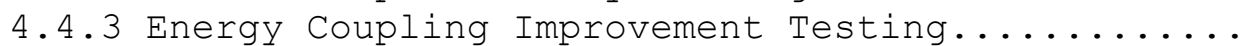

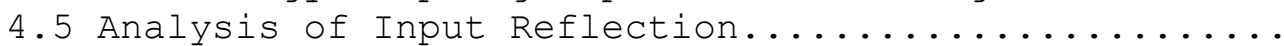

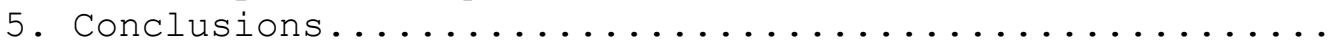

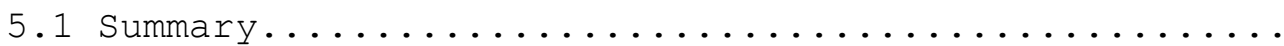

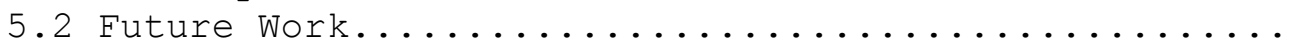

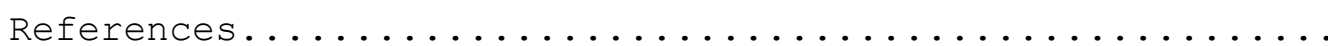
APPENDICES

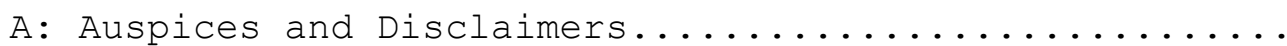

B: Phase and Magnitude Sample Results for Different

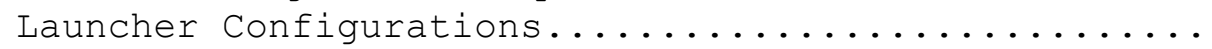

C: $\mathrm{R}^{2}$ and Д Results for Different Launcher

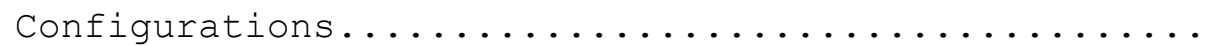


Page

Table 4.1: Waveguide Impedances Inside of the Structure, calculated from Equation 4.4. Used in Equations 4.5 and 4.7 to calculate input reflection into the Teflon.... 16

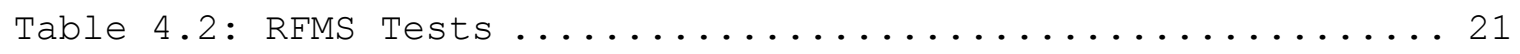

Table 4.3: Correlation coefficients between average |S11|

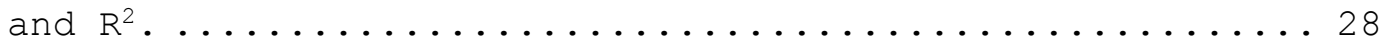

Table 4.4: Comparing the of frequencies with metrics above the threshold in each analysis technique........ 37

Table 4.5: This is a selection of the wider bands that have $\mathrm{R}^{2}>0.90$ which should be referenced before choosing a particular test frequency for highest odds of success in an experiment with that frequency. Shown are band start and stop frequencies and band widths.......... 37 
LIST OF FIGURES

Page

Figure 1.1: Detonation Measurements, Ionization Pin

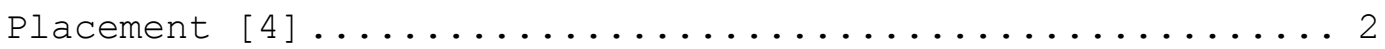

Figure 1.2: Manganin Gauge Placement Between Comp B Layers

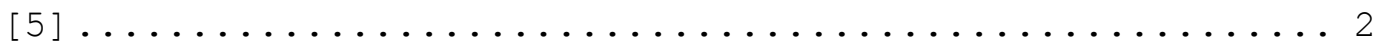

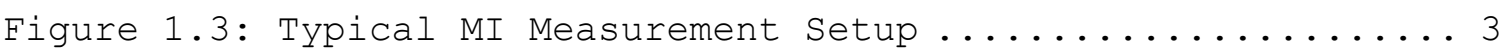

Figure 3.1: Interferometer Diagram; The synthesized RF

signal (top) drives the antenna (attached to test

unit). The return signal is applied to a circulator,

filter, and mixer with LO input to produce the REF

signal; LO is mixed with RF directly, filtered, and

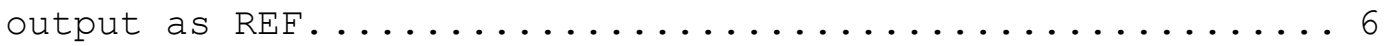

Figure 3.2: TATB experiment reference and return signals, showing phase and amplitude differences between

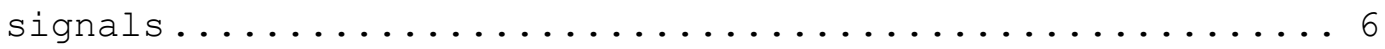

Figure 3.3: Baseband signals unfiltered after mixing the reference and return signals as in Equation $3.3 \ldots \ldots \ldots .8$

Figure 3.4: Real and Imaginary Doppler signals, without DC offset removal or high-pass filtering to remove low-

frequency components..................... 8

Figure 3.5: Unwrapped phase from the complex vector

comprised of the real and imaginary signals in Figure

3.4 after DC offset removed. Two data markers show the

most linear portion of the unwrapped phase used to

calculate the detonation velocity from the experiment.... 9

Figure 4.1: Ring Fixture Measurement System (RFMS) Top-Down

View. Novel test apparatus measured in this paper.

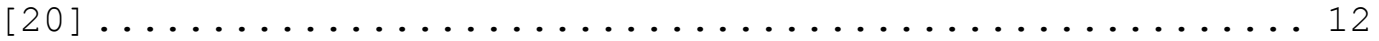

Figure 4.2: Expanded View of the RFMS. Not shown are additional waveguide mounts used. Critical components include the 'Solid Dielectric, Teflon', 'Target Reflector, $A L$ 6061-T6', 'Axle, $A L$ 6061-T6', and 'Housing, SST 304', which compose the circular geometry explored in this experiment $[20] \ldots \ldots \ldots \ldots \ldots$

Figure 4.3: RFMS Launcher Angles. Manufactured at $60^{\circ}$ and

$72^{\circ}$. Bottom of RFMS from Figure 4.1 shown............ 14

Figure 4.4: Three Material Reflection Diagram. Transitions

from wax into air, and into the Teflon ring. These

transitions motivate filling the launchers with wax to

couple more energy into the Teflon................ 17

Figure 4.5: Solidifying Paraffin Wax Dielectric

Measurements: Measurements made at 0 (blue), 19 (red), 
and 29 (green) minutes after application to probe region. The constant varies between 2.112 and 2.377 between $15 \mathrm{GHz}$ and $40 \mathrm{GHz}$. This constant is used for Region 1 in Figure 4.4 to determine increase in energy

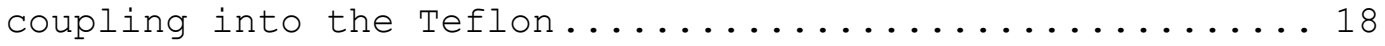

Figure 4.6: Wax-filled waveguide reflection improvement: $0.1,0.5,1.0 \mathrm{~mm}$ air gap. With worst case $\varepsilon_{\mathrm{r}}$ of 1.5, there is improvement over no wax for Region 2 air gaps

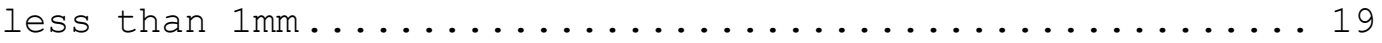

Figure 4.7: Compiled Results Example. Conversion from frequency swept phase at one TRA to a single frequency

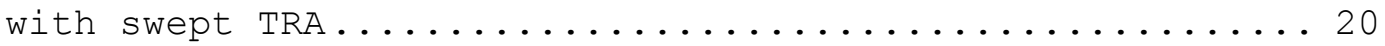

Figure 4.8: Input Reflection should ideally be $0 \mathrm{~dB}$, however, the magnitude varies greatly with frequency between $-5 \mathrm{~dB}$ and $-20 \mathrm{~dB}$, with some frequencies as low as $-50 \mathrm{~dB}$. From Test ID \#19, S11 over $26 \mathrm{GHz}-40 \mathrm{GHz}, 0^{\circ}$

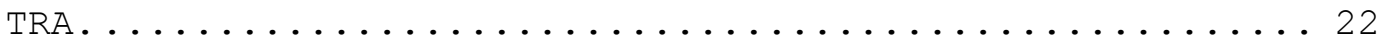

Figure 4.9: |S11| over one rotation of TRA at $26 \mathrm{GHz}$. Input Reflection should ideally be $0 \mathrm{~dB}$, however, the magnitude varies largely with frequency between $-5 \mathrm{~dB}$ to $-20 \mathrm{~dB}$, with some points as low as $-30 \mathrm{~dB}$. From Test

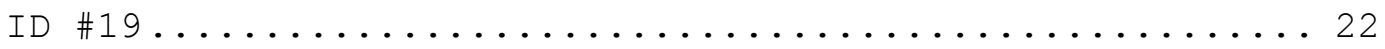

Figure 4.10: Input Reflection phase should ideally be a linear change vs. TRA, which would appear as a sawtooth waveform. Shown here, the phase changes rapidly and does not wrap, showing non-ideality from the expected phase change. From Test ID \#19 LS11 of Launcher 1 over one rotation of the TRA, no wax, at

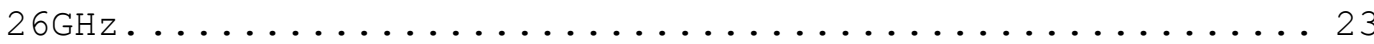

Figure 4.11: Comparison in phase between consecutive runs for Tests \#18 and \#19 at 26GHz The strong similarity in phase measurements indicate repeatability between phase measurements, allowing for some time of

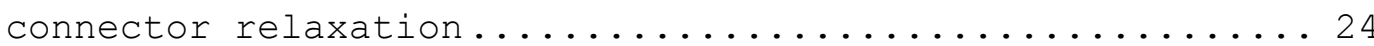

Figure 4.12: Phase Vs. TRA for compiled results and CW, which are subjectively identical indicate that the method of compiled phase points yields identical results to a CW measurement. CW sweep phase vs compiled results phase at $26 \mathrm{GHz}$ in Launcher 1, no wax

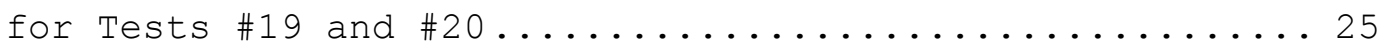

Figure 4.13: Identical Configuration Comparisons Between $\mathrm{R}^{2}$ Metric in Four Cases, Tests \# 16, 17, 18 and 19. The difference in $\mathrm{R}^{2}$ between any two tests is less than $1 \%$ for 75\% of the frequencies. This indicates an acceptable amount of repeatability in testing......... 26 
Figure 4.14: Variance in S11 magnitude vs. frequency between $26 \mathrm{GHz}$ and $28 \mathrm{GHz}$ between the waveguide filled with wax and without wax from Tests \# 19 and 25. Variance in the magnitude increased at some frequencies and decreased at some frequencies in the overlapping frequency range between the two configurations. The effect of filling the launchers with wax is hard to determine if it helped overall..... 27

Figure 4.15: Variance in $|S 11|$ between Launchers 1 and 2 without wax. There is no constant increase or decrease in variance over the range $26 \mathrm{GHz}$ and $40 \mathrm{GHz}$, so it is hard to tell if the launch angle variation helps the

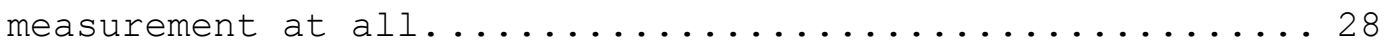

Figure 4.16: |S11| mean from all launchers 26-40GHz. Launchers with wax had higher $|S 11|$, indicating more

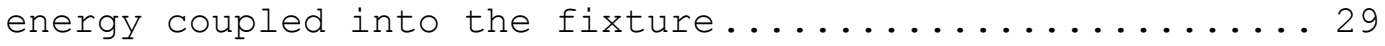

Figure 4.17: Average |S11| taken over all TRA for each frequency vs. $R^{2}$. Correlation coefficient between average $|S 11|$ and $R^{2}$ is $\rho=-0.8109$, indicating that signals with better linear phase have lower |S11|

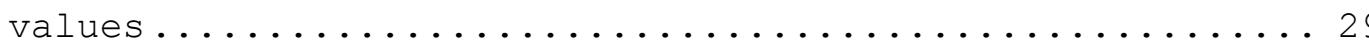

Figure 4.18: Top left: ideal unwrapped phase, over $360^{\circ}$ TRA, unwrapped from $0^{\circ}$ to $-360^{\circ}$; top right: ideal phase from top left, wrapped to between $-180^{\circ}$ and $180^{\circ}$, black dots shown are the detected zerocrossings; bottom left: ideal wrapped phase, black dots shown are the actual crossings, red markers shown are the expected crossings in the process of being correlated with the actual crossings (the markers are not fully correlated with the actual crossings in this figure, and will yield a lower error-per-point if shifted to the right) bottom right: ideal wrapped phase with white noise added (to model overmoded nature and non-ideality in actual phase return). If the signal is noisy, extra zero-crossings will appear. However, the method deals with this issue by only correlating the closest zero-crossing with the

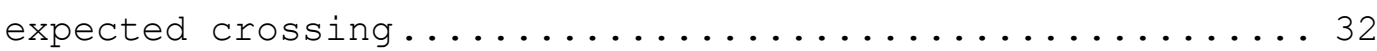

Figure 4.19: $\mathrm{R}^{2}$ : a linearity metric of 1 indicates ideal phase, and a linearity metric of 0 indicates no phase unwrapping. Frequency bands between $\sim 33 \mathrm{GHz}-35 \mathrm{GHz}$ and $37 \mathrm{GHz}-38 \mathrm{GHz}$ have higher overall phase linearity and

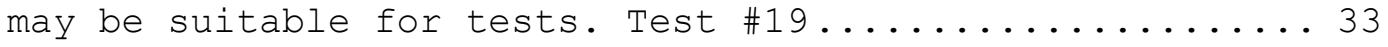
Figure 4.20: Results of zero-crossings tests. Д indicates how frequently the phase crossed zero to when it 
should, indicated periodicity in the phase. The results do not vary as much as the $\mathrm{R}^{2}$ method. For Test

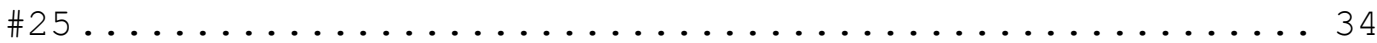

Figure 4.21: The best frequency indicated in Figure 4.20 actual phase. A high value from the zero-crossings method does not necessarily indicate linear phase unwrapping, but indicates periodic nature within the

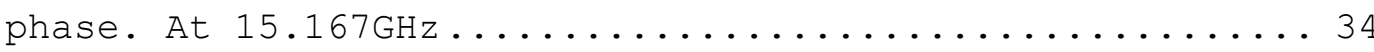

Figure 4.22: Wrapped phase example of a good point from

Figure 4.19, at 39.1978GHz. The $\mathrm{R}^{2}$ method metric indicates a high amount of phase linearity.......... 35

Figure 4.23: Left: unwrapped phase example of a good frequency, 39.1978GHz, from Test \#19. Right: phase of a frequency with larger $R^{2}$ metric of 0.9152 when unwrapped has a jagged and irregular pattern. Therefore the threshold for the of good frequencies

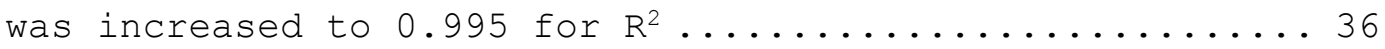

Figure 4.24: $R^{2}$ metric compared to Д metric; low frequencies indicated by dark blue and higher frequencies are

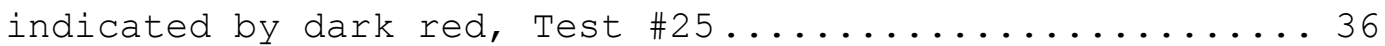

Figure A.1: Magnitude of S11 vs. TRA at $39.1978 \mathrm{GHz}$ for Test

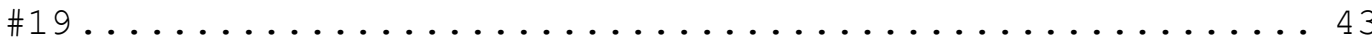

Figure A.2: S11 Phase Vs. TRA at 39.1978GHz for Test \#19 ....43 Figure A.3: Magnitude of S11 vs. TRA at 39.1978GHz for Test

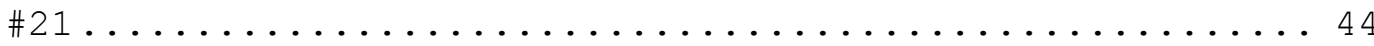

Figure A.4: S11 Phase vs. TRA at 39.1978GHz for Test \#21 .... 44 Figure A.5: Magnitude of S11 vs. TRA at $39.1977 \mathrm{GHz}$ for Test

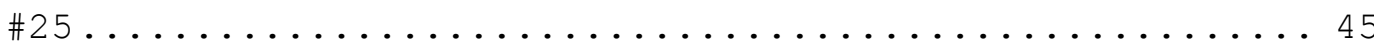

Figure A.6: S11 Phase Vs. TRA at 39.1977GHz for Test \#25 .... 45

Figure A.7: Magnitude of S11 vs. TRA at $39.1977 \mathrm{GHz}$ for Test

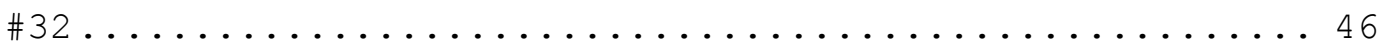

Figure A.8: S11 Phase Vs. TRA at $39.1977 \mathrm{GHz}$ for Test \#32 ....46

Figure A.9: Magnitude of S11 vs. Frequency at $0^{\circ}$ TRA for

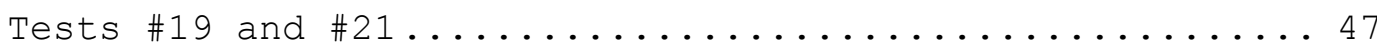

Figure A.10: Magnitude of S11 vs. Frequency at $45^{\circ}$ TRA for

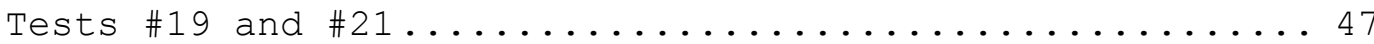

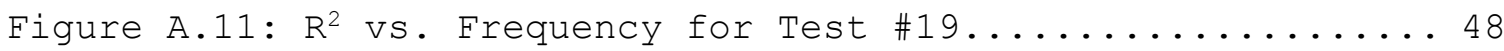

Figure A.12: $R^{2}$ Vs. Frequency for Test $\# 21 \ldots \ldots \ldots \ldots \ldots \ldots \ldots \ldots$

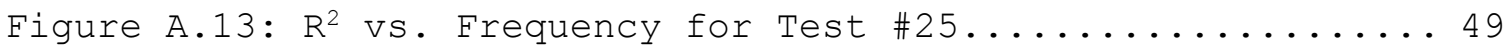

Figure A.14: Д vs. Frequency for Test \#25 ............... 50

Figure A.15: $R^{2}$ vs. Frequency for Test $\# 32 \ldots \ldots \ldots \ldots \ldots \ldots \ldots \ldots$

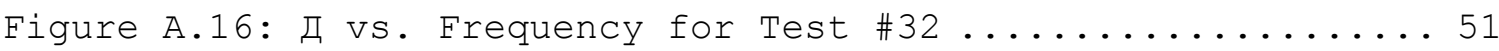


Equation (3.1): Reference Signal Model.............. 5

Equation (3.2): Return Signal Model................. 5

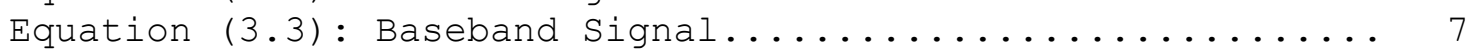

Equation (3.4): Doppler Signal.................. 7

Equation (3.5): Quadrature Sine (Q) Generation............ 7

Equation (3.6): Quadrature Cosine (I) Generation.......... 7

Equation (3.7): Complex Vector Addition................ 9

Equation (3.8): Phase Extraction from Complex Vector....... 9

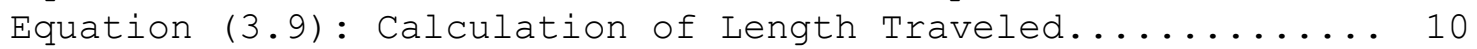

Equation (3.10): Detonation Velocity Calculation........... 10

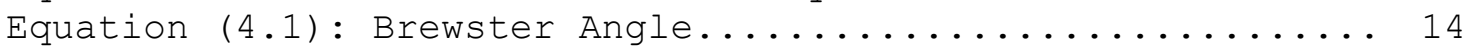

Equation (4.2): Rectangular Waveguide Cutoff Frequency...... 15

Equation (4.3): Fundamental Frequency Cutoff Frequency...... 15

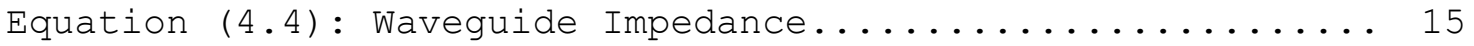

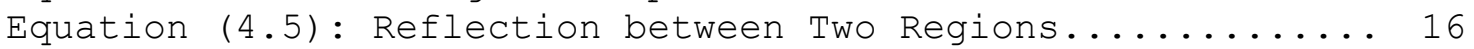

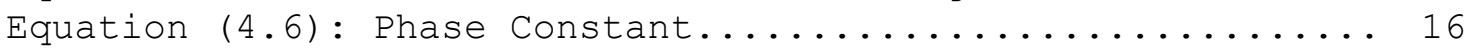

Equation (4.7): Three Material Region Input Reflection...... 16

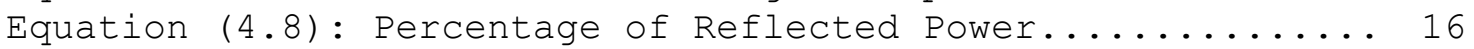

Equation (4.9): Д Metric Definition.................. 30 


\section{LIST OF ACRONYMS}

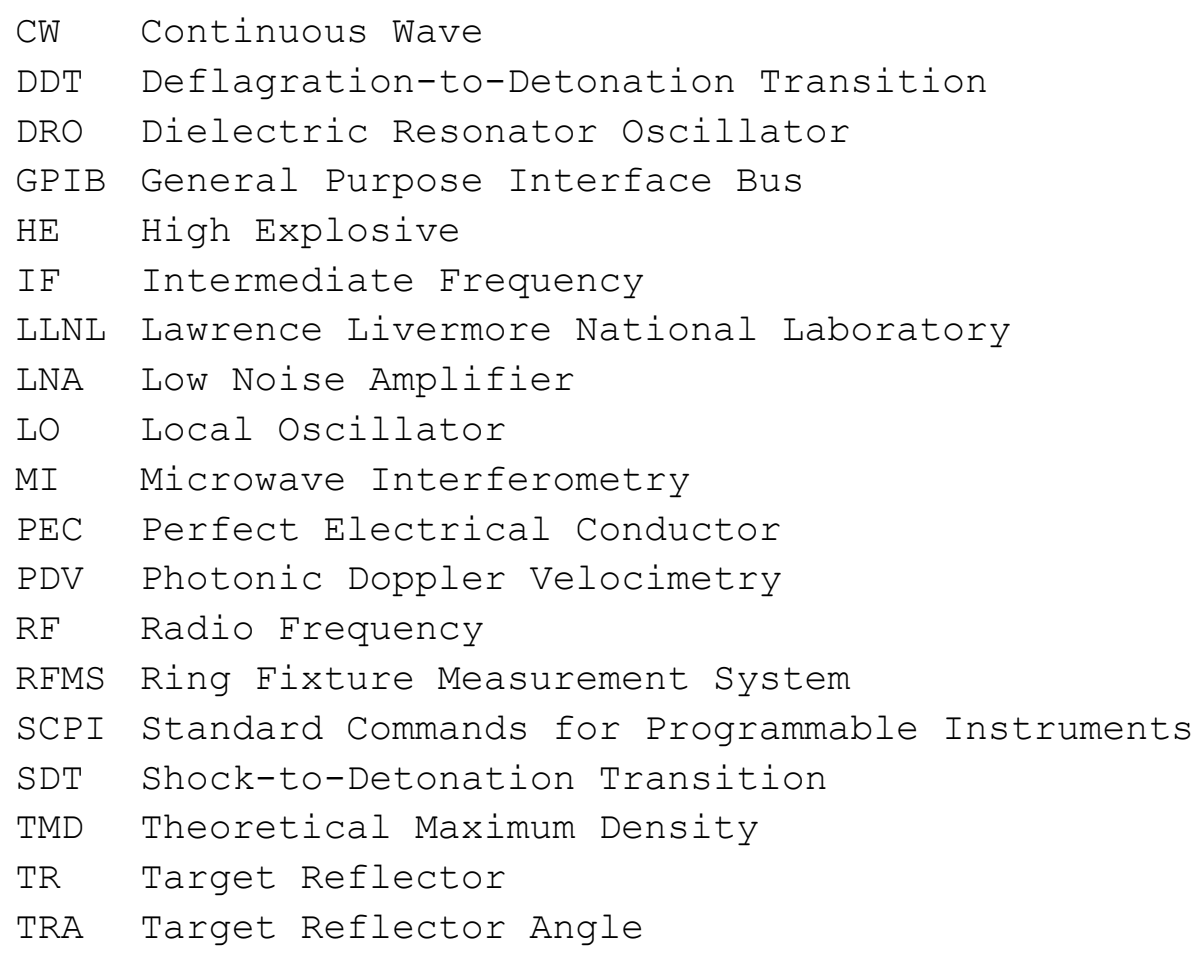




\section{Introduction}

\subsection{Background}

Lawrence Livermore National Laboratory (LLNL) was established in 1952 with the goal of being a laboratory to develop "new ideas" to compete against Los Alamos National Laboratory [1]. This paper continues this tradition: Microwave Interferometry (MI) development for high explosives experiments. In this paper, a novel explosive structure is analyzed for use in an MI experiment. A characteristic of high explosives (HE) is detonation velocity, which is the theoretical velocity at which the detonation wave propagates through an explosive.

Detonations can initiate from shock (impact) or deflagration (burning) processes, via shock-to-detonation (SDT) or deflagration-to-detonation transitions (DDT) respectively. HE detonation involves detonation wave initiation at one end of a cylinder, wave propagation to the other end, and $\mathrm{HE}$ reaction and explosion. This detonation wave is highly ionized (there is a dense concentration of ionized particles) [2] and theoretically moves with a constant velocity. This is my best understanding of the physical process, but I do not have a specific reference for the reader to view to understand the process.

MI involves high-frequency signal transmission and reflection measurement [3]. The Doppler frequency shift of the return signal is converted to the velocity of moving reflective surfaces. Highly ionized material (the detonation front) is reflective to microwave signals, while un-reacted $\mathrm{HE}$ is transparent. This permits tracking of the detonation front along the full length of an $\mathrm{HE}$ sample from a single location.

MI is non-intrusive (negligible interference) and higher resolution than existing standards with continuous measurements instead of discrete points [3]. Since MI is a continuous measurement, MI can measure changes in the experiment on a short time scale, such as possible changes in detonation velocity due to discontinuities in the explosives.

\subsection{Project Motivation}

Explosives measurement standards include ionization pins [4], Manganin gauges [5], and Photonic Doppler Velocimetry (PDV) [6]. Ionization pins are pins that output a signal depending on the quantity of ionization around the probe. The highly ionized

detonation front is detected by ionization pins at discrete locations, which can disturb the measurement (see Figure 1.1). Manganin gauges (discrete location pressure measurement) change resistance when compressed by the detonation front and are designed 
to minimize wave propagation interference [7]. However, Manganin gauges are discrete measurements of detonation position. Manganin gauges have been used in previous detonation measurements, as shown in the test geometry in Figure 1.2. PDV probes, an interferometric light-based measurement system, measure cylinder expansion at the detonation wave location by Doppler shift in the light signal. The PDV measurement is an indirect detonation front measurement and is recorded outside the $\mathrm{HE}$ container. Each probe represents a data point in an explosive measurement. Each probe requires oscilloscope measurements and supporting hardware.

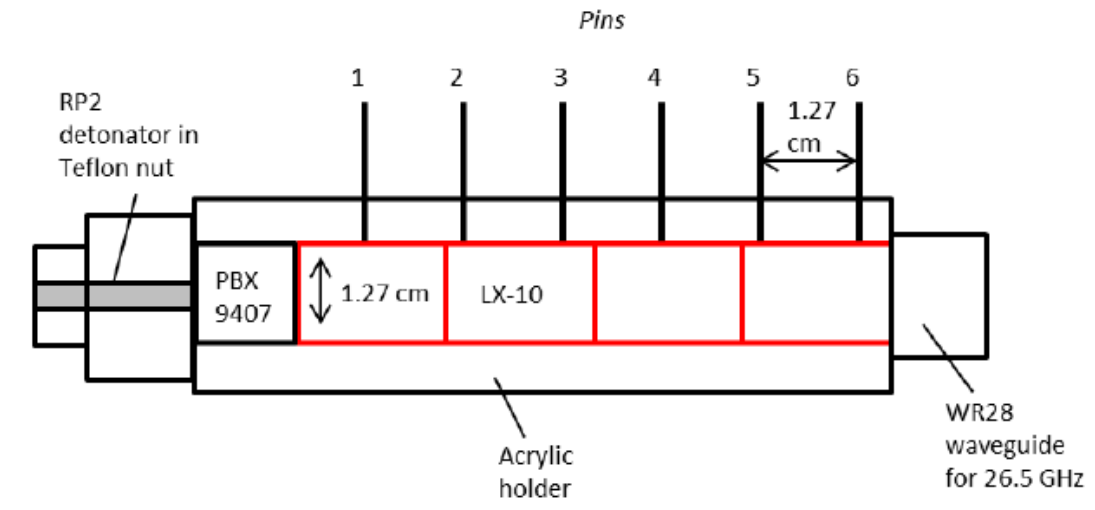

Figure 1.1: Detonation Measurements, Ionization Pin Placement [4].

Non-intrusive and continuous MI measurements provide high (10's of picoseconds) temporal and 1D spatial resolution (millimeters). Figure 1.3 shows a typical MI experiment set-up; waveguide attached to an HE-filled cylinder.

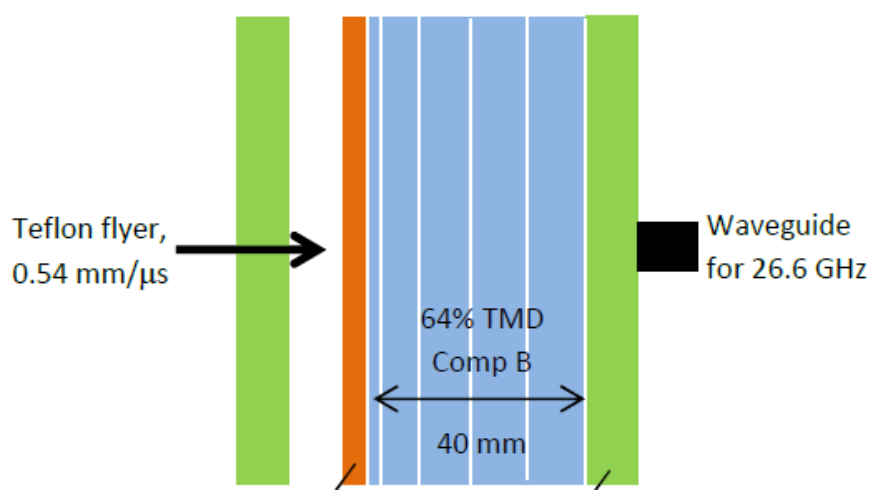

Figure 1.2: Manganin Gauge Placement Between Comp B Layers [5]. 


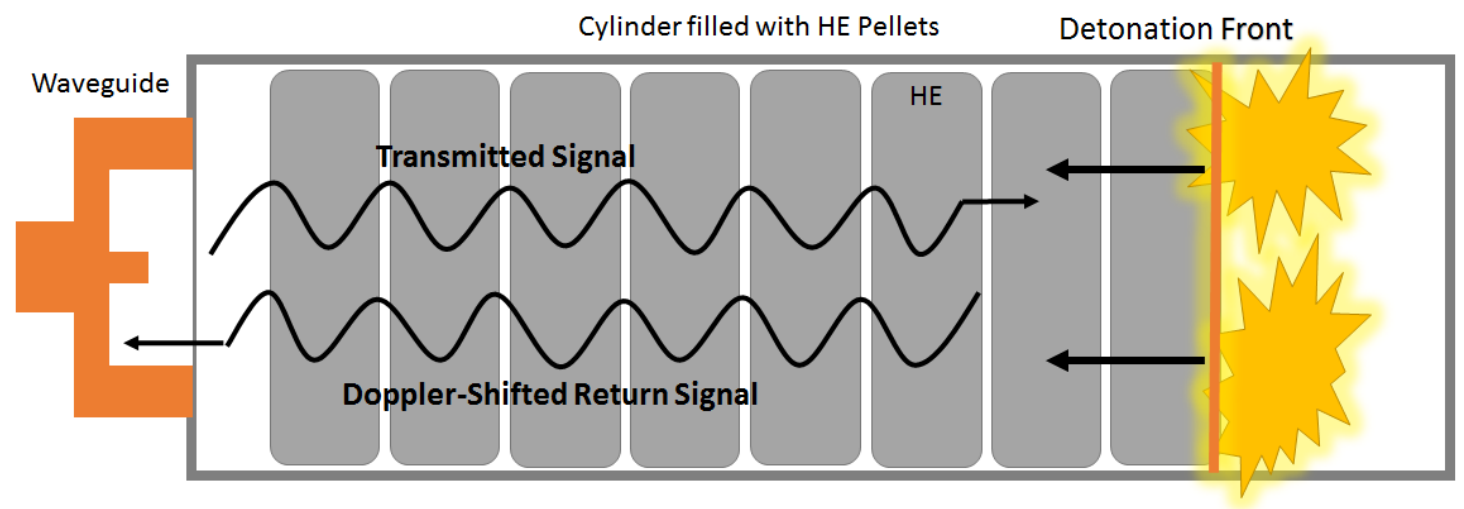

Figure 1.3: Typical MI Measurement Setup.

\subsection{Scope}

In Chapter 2, I present a comprehensive literature review of existing work in the field of MI as it applies to explosives with some review of other applications of MI.

In Chapter 3, I demonstrate an existing analysis of a cylindrical tube HE experiment to show the MI processing technique. This geometry is the only one explored to date to the best of my knowledge. The same processing used in this geometry may be used in the novel geometry that I measure and analyze in Chapter 4. I provide the basis of why a linearly changing input reflection phase is desired.

In Chapter 4, I present a novel geometry for MI experimentation. My contributions to the field include measurement of the system, analysis of the results with two different metrics, and determination of a good frequency to use with this structure. The system presented in Chapter 4 is a simulated system built with hardware, and did not use any explosives.

In Chapter 5, I present a summary of the work performed in the paper and recommend improvement for future work. 


\section{Early Work on MI}

An early example on MI for explosive applications was written in 1954 at the University of Utah [2]. The authors note that detonating explosives produce a high ionization density wavefront approximated as a perfect electrical conductor ( $P E C)$, which is reflective to microwave radiation.

NASA performed experiments measuring gas in an expansion tube [8]. Long shock tube velocity measurements required extensive electrical measurement equipment, which could be replaced by MI methods.

Los Alamos National Lab experiments compare MI to streak camera and ionization pin measurements [9] to quantify MI advantages. Microstrip cable is inserted into the $\mathrm{HE}$, causing electrical microwave signal reflections, rather than a conducting detonation front causing electromagnetic microwave signal reflection

Additional MI work includes a US Army detonation study [10]. MI measurements quantify shock waves in reactive and unreactive porous materials [11, 12]. Other MI studies include unstable detonations and shocked air, shock wave displacement, and detonation process measurements [13-15]. Another MI study observes detonation velocity changes in the interface between explosives [3]. Other recent uses of MI include measuring detonation fronts and run-to-detonations [16] .

LLNL MI methods includes phase-based quadrature analysis. The phase-based approach is explained in an unpublished work by R. Kane [17].

Previous LLNL MI work includes SDT and DDT transition experiments on layered porous explosives to measure ignition and reaction growth [5]. LLNL has performed MI on expanding ionized plasma plumes to model DDT and SDT detonation evolution [18].

MI has also been used in breast cancer imaging at other institutions [19]. 


\section{MI for One-Dimensional High Explosives}

The data presented in this chapter was collected from a previous experiment on a cylindrical tube of HE. This chapter is presented as an example analysis of data processing after an experiment is performed, since this technique would be used in Chapter 4 on the novel geometry. I also justify in this chapter why a linearly changing phase response is desired from the return signal.

\subsection{Analysis}

A basic LLNL interferometer diagram appears in Figure 3.1. Dielectric Resonator Oscillators (DRO) output the Radio Frequency $(R F)$ and local oscillator (LO) signals for mixing down to an intermediate frequency (IF). The RF oscillator applies a signal to a directional coupler and circulator, which drives the antenna. The return signal from the antenna is bandpass filtered and mixed down to an IF, bandpass filtered again, passed through an LNA, and then is output as the return (RET) signal. The output signal from the directional coupler is mixed with the LO, bandpass filtered, amplified, and then output as the reference (REF) signal.

What follows is a description of the math involved with the phasebased analysis on MI to demonstrate the desired results that will be analyzed in the novel structure in chapter IV. The following analysis example describes a TATB (a type of explosive) experiment. The $26.5 \mathrm{GHz}$ transmit signal is downconverted in the interferometer to $255 \mathrm{MHz}$, and then sampled at $25 \mathrm{GS} / \mathrm{s}$.

Interferometer waveforms include the reference $R F$ ( $S_{\text {Ref }}$ ) and return ( $\left.\mathrm{S}_{\text {Ret }}\right)$ signals defined by Equations 3.1 and 3.2, respectively.

$$
\begin{aligned}
& S_{\text {Ref }}=A \cos (2 \pi f t) \\
& S_{\text {Ret }}=B \cos (2 \pi f t+\theta+\phi(t))
\end{aligned}
$$

where $\theta$ is a constant phase offset from the reference signal and $\varphi$ is the Doppler shift in the signal [17]. The $\theta$ term is removed since it has no signal processing effects [17].

Figure 3.2 shows the reference and return signals between $40.000 \mu \mathrm{s}$ and $40.002 \mu \mathrm{s}$ after the detonation trigger. 


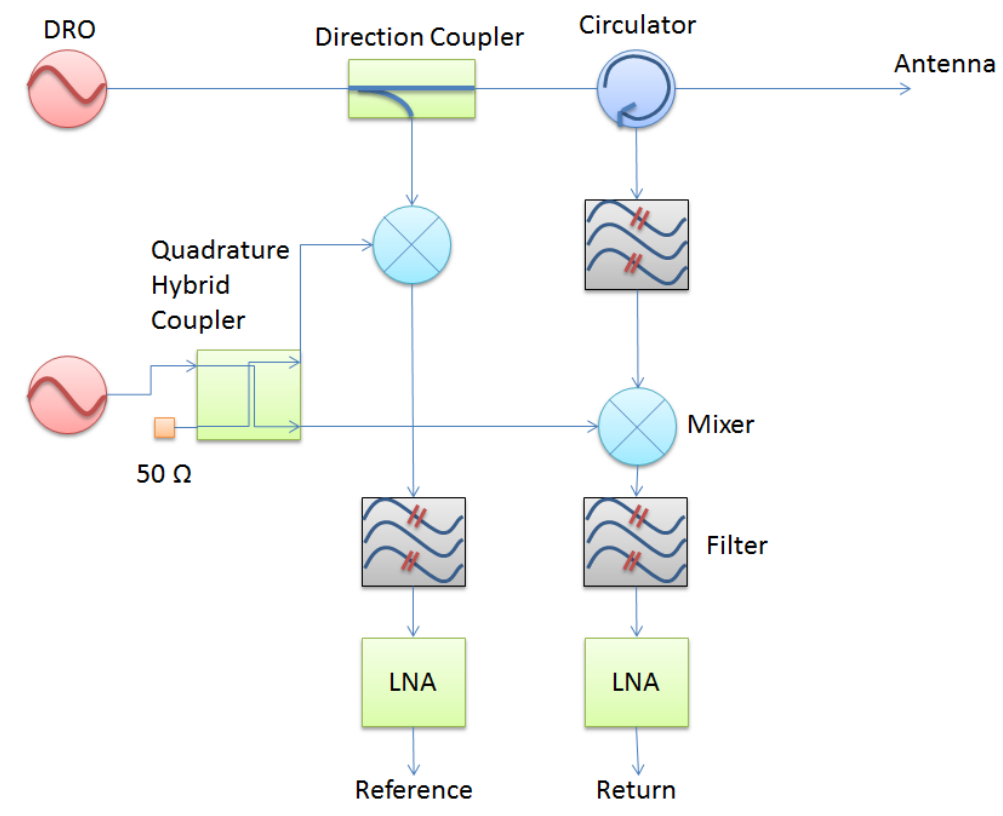

Figure 3.1: Interferometer Diagram; The synthesized RF signal (top) drives the antenna (attached to test unit). The return signal is applied to a circulator, filter, and mixer with LO input to produce the REF signal; LO is mixed with RF directly, filtered, and output as REF.

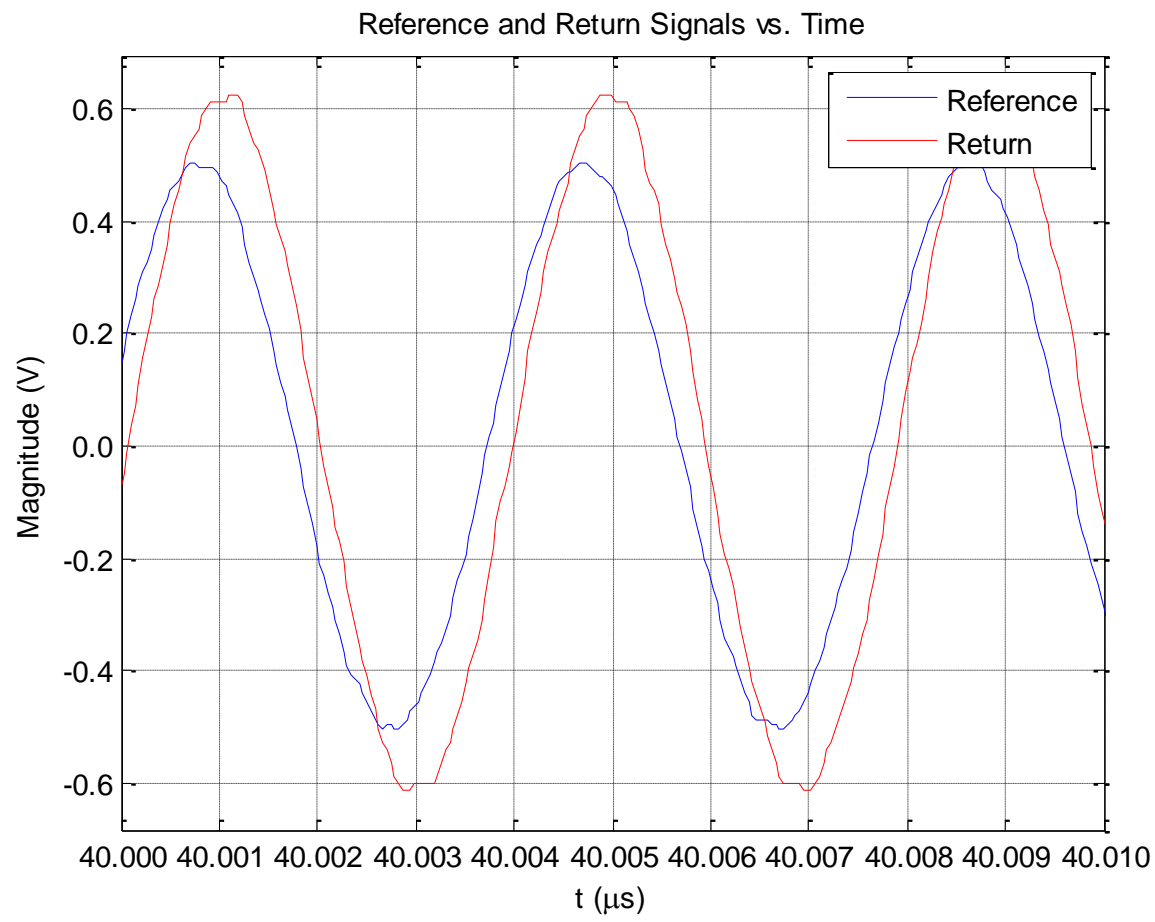

Figure 3.2: TATB experiment reference and return signals, showing phase and amplitude differences between signals. 
Each signal is mixed down to an IF of $255 \mathrm{MHz}$, bandpass filtered about the IF, and recorded by the oscilloscope. Signals are digitized; the IF signal is bandpass filtered in software. The IF signals are mixed to produce the baseband signal. Equation 3.3 shows reference and return signal mixing.

$$
S_{\text {baseband }}=S_{\text {Ref }} \cdot S_{\text {Ret }}=\frac{A B}{2}\{\cos [4 \pi f t+\phi(t)]+\cos [-\phi(t)]\}
$$

Figure 3.3 shows reference and return signal mixing to produce the baseband signal.

The baseband signal is low-pass filtered to remove high-frequency residuals from the mixing process, to remove high-frequency noise, and to extract the Doppler signal of interest. Low-pass filtering the results in the Equation 3.4 signal.

$$
S_{\text {Doppler }}=\cos [\phi(t)]
$$

To extract the phase from the Doppler signal, quadrature (real and imaginary) signals are synthetically generated using the reference signal as a 'cosine' and time shifting the reference into a 'sine' using Equations 3.5 and 3.6. Also, see Equations 3.8-3.10.

$$
\begin{gathered}
S_{\text {sin }}=\operatorname{angle}\left(S_{r e f}\right)+90^{\circ} \\
S_{\text {cos }}=S_{\text {ref }}
\end{gathered}
$$

The mixed products from using Equations 3.5 and 3.6 in Equation 3.3 are shown in Figure 3.3. 


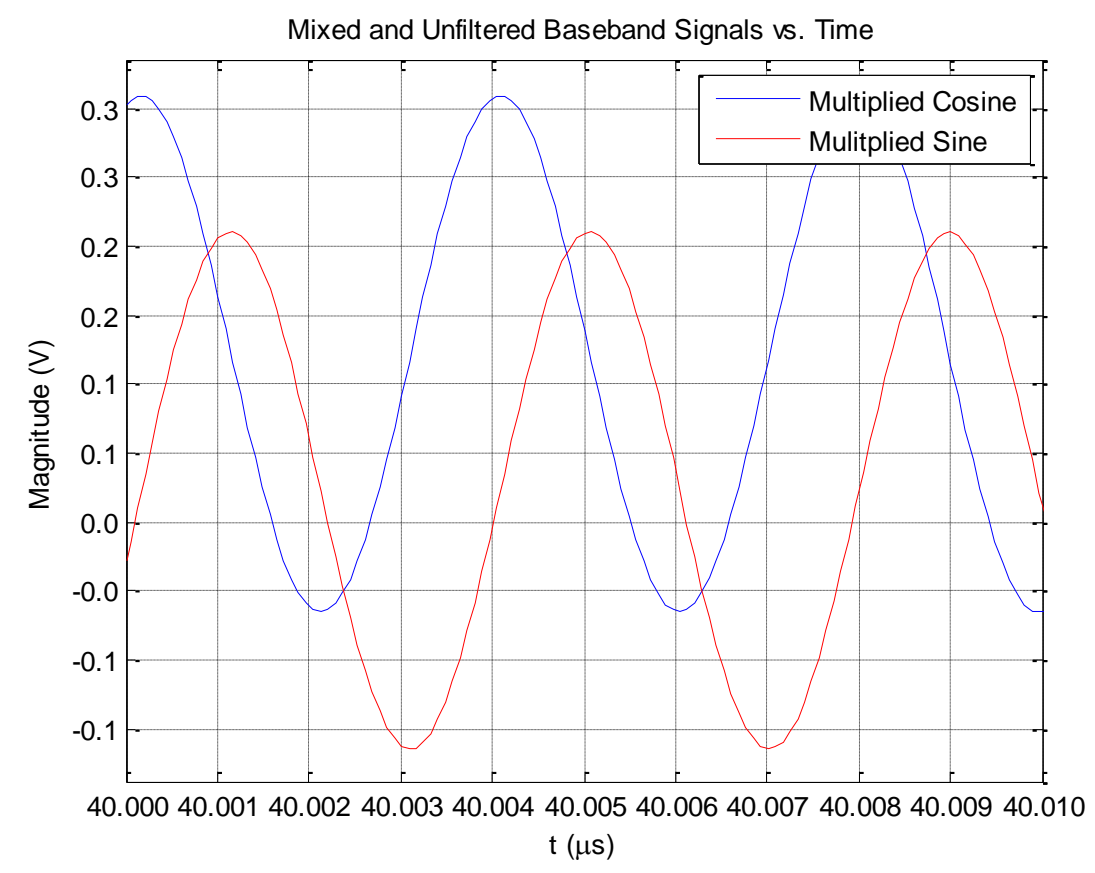

Figure 3.3: Baseband signals unfiltered after mixing the reference and return signals as in Equation 3.3 .

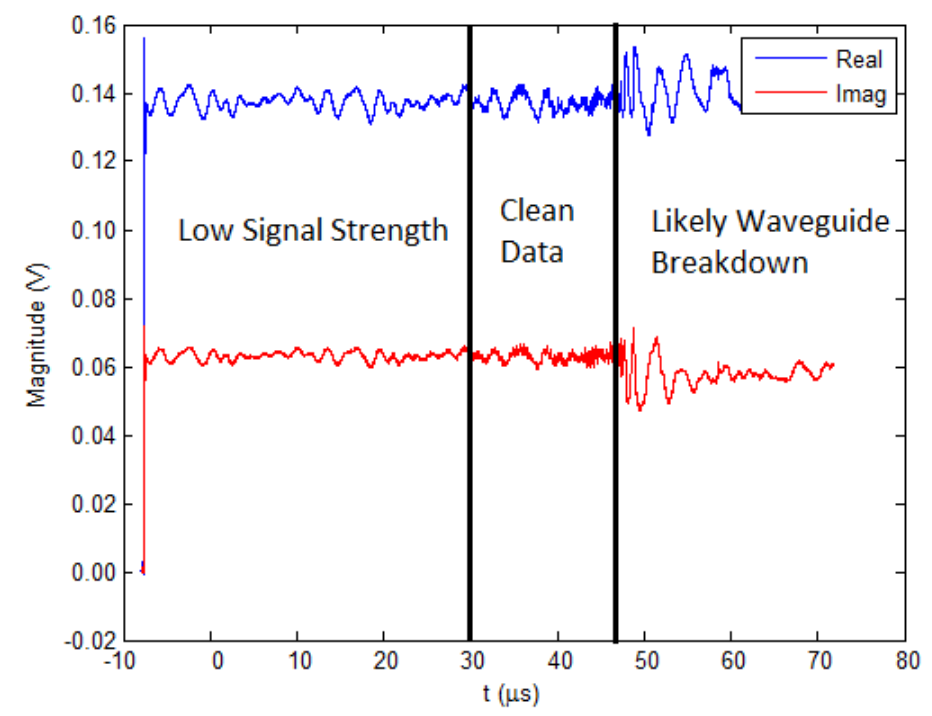

Figure 3.4: Real and Imaginary Doppler signals, without DC offset removal or high-pass filtering to remove low-frequency components.

The 'cosine' and 'sine' are mixed with the return signal to calculate baseband signals as in Equation 3.3 to get "real" and "imaginary" signals, respectively. These signals are then highpass filtered to remove DC offset and low-frequency products lower 
than the Doppler shift frequency (about $10 \mathrm{MHz}$ for detonation velocity measurements). Figure 3.4 shows the Doppler real and imaginary signals before DC offset removal. These signals are highpass filtered and are added into a complex-valued vector using Equation 3.8 .

$$
S_{\text {complex }}=S_{\text {real }}+j \cdot S_{\text {imaginary }}
$$

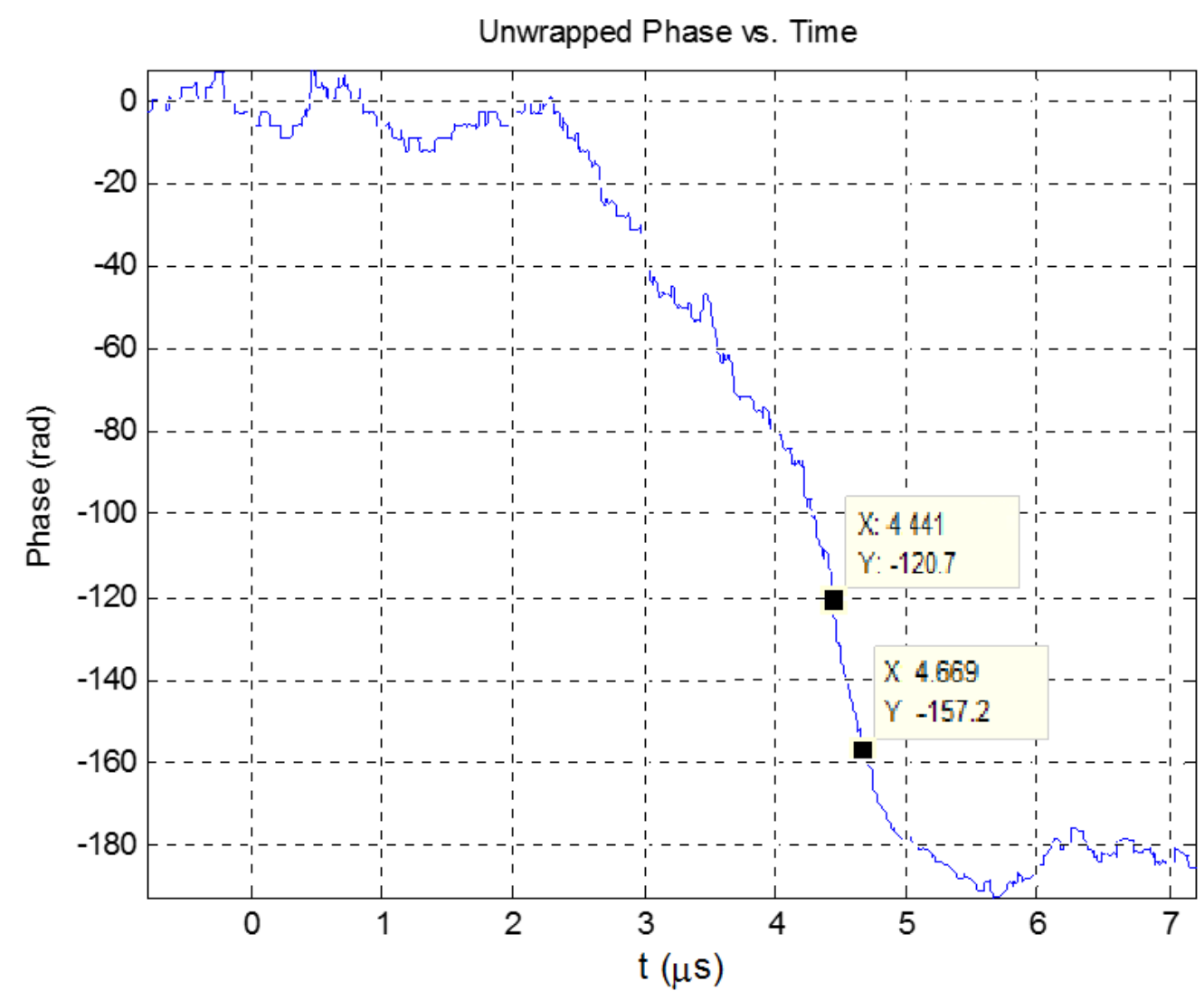

Figure 3.5: Unwrapped phase from the complex vector comprised of the real and imaginary signals in Figure 3.4 after DC offset removed. Two data markers show the most linear portion of the unwrapped phase used to calculate the detonation velocity from the experiment.

The phase of the complex vector is calculated using Equation 3.8 and is then unwrapped to show continuous changes in phase.

$$
\phi=\tan ^{-1} \frac{S_{\text {imaginary }}}{S_{\text {real }}}
$$

Since the detonation wave moves $\lambda / 2$ every $2 \pi$ change in measured phase, then the detonation front $1 / 2$ the change in phase scaled by $2 \pi$, multiplied by the wavelength in the medium $\left(\lambda_{g}\right)$ given by Equation 3.9. 


$$
\Delta l=\left(\frac{1}{2}\right) \cdot \frac{\Delta \phi}{2 \pi} \cdot \lambda_{g}
$$

Detonation velocity is calculated using the distance from Equation 3.9 divided by the time traveled, given in Equation 3.10. The slope of the unwrapped phase from Equation 3.8 [17] is scaled to approximate the velocity.

$$
\text { velocity }=\frac{\Delta \phi}{4 \pi \Delta t} * \lambda_{g}
$$

The wavelength of the transmitted signal inside of the explosive $\left(\lambda_{g}\right)$ is used in Equation 3.10, since the Doppler phase shift is dependent on $\lambda$ at the detonation front.

Assuming a dielectric constant of the explosive $\varepsilon_{r}=4$, using the two data cursors shown in Figure 3.5 and using Equation 3.10, the detonation velocity is calculated to be $7.18 \mathrm{~mm} / \mu \mathrm{s}$. This is close to the average $7.66 \mathrm{~mm} / \mu \mathrm{s}$ that comes from PDV probe data used in the experiment. There has been a significant amount of other analysis steps taken to match the detonation velocity from MI to PDV probe data. Even in the simplified analysis presented above, many subjective decisions need to be made, including filter cutoff frequencies for the initial bandpass filter and low-pass filter after mixing, and the choice to remove the mean of the signal to remove DC components from the baseband signal. Wavelet analysis should be explored to remove many of these subjective decisions.

This is the overall approach used on data for MI experiments, and would be the type of analysis performed on data taken from an experiment involving a circular geometry, which will be explored in the next chapter. Ideally the phase results are linear, like the data chosen in between the two cursors in Figure 3.5. Linear phase results shows constant detonation velocity, which would match theory. 


\section{MI for Circular Geometry}

Structures different from the standard cylindrical tube are of interest to LLNL for MI experiments. Also of interest is the case of overmoded structures, which means that higher order TEM modes can propagate in the system, which make the phase response difficult, or impossible to predict. This information is of academic interest. The system presented in this chapter had been manufactured for my project. My contribution to the field is measuring and analyzing this structure to determine usable frequencies and to view the results.

The structure used in this chapter contained no explosives, and is meant to simulate in hardware the input phase results that we would get if the structure were manufactured with explosives for an actual experiment.

\subsection{Structure}

A geometry of interest to LLNL for an explosive measurement is a circular ring as shown in Figure 4.1. The Ring Fixture Measurement System (RFMS) was manufactured to experimentally measure the S11 parameter of the system as a function of a target reflector's angle in a circular geometry. The input port to the system is a coax to waveguide adapter (shown in green on the bottom of Figure 4.1). This adapter transmits a microwave signal into a Teflon cylinder (completely encased in metal) that has a target reflector along its height. The target reflector mimics the moveable detonation front of an explosion. This entire system is designed to mimic the geometry of an explosive measurement set-up. S11 is measured because in an explosive measurement (as shown in the previous chapter) a single antenna is used for both transmit and receive in the experiment. Therefore, the measurements are of the input reflection into the system. To characterize how this novel structure will perform at microwave frequencies, the input reflection of the structure (S11) needs to be characterized. 


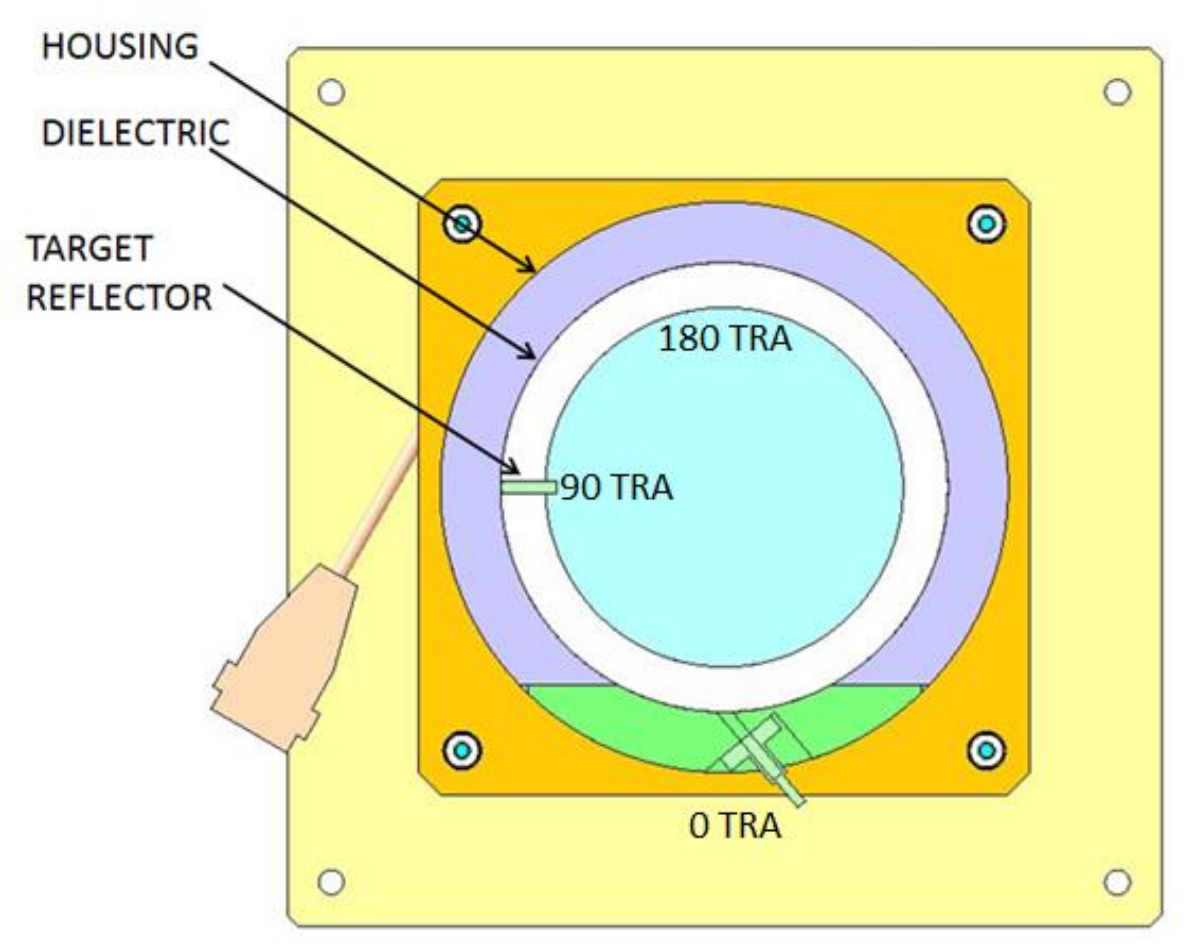

Figure 4.1: Ring Fixture Measurement System (RFMS) Top-Down View. Novel test apparatus measured in this paper [20].

The composition and set-up of the RFMS is shown in Figure 4.2. A housing (yellow and purple in Figure 4.2) mounts onto a motor on a baseplate. In the housing is a Teflon dielectric material. The Teflon mimics explosive material in the RFMS. Inside the Teflon along its height is an aluminum bar (target reflector, or TR), which mimics a detonation front. An axle mounts onto the motor and supports the Teflon ring, allowing it to rotate, which simulates movement of the detonation front. The target reflector angle (TRA) is the angle between the waveguide adapter and the TR. A lid caps the fixture so that the TR is completely within metal, forming a reflective front in a wrapped waveguide. A slot was manufactured into the housing to hold a waveguide mount (green in Figure 4.1). Mounts were made to transmit the signal into fixture at two angles. 


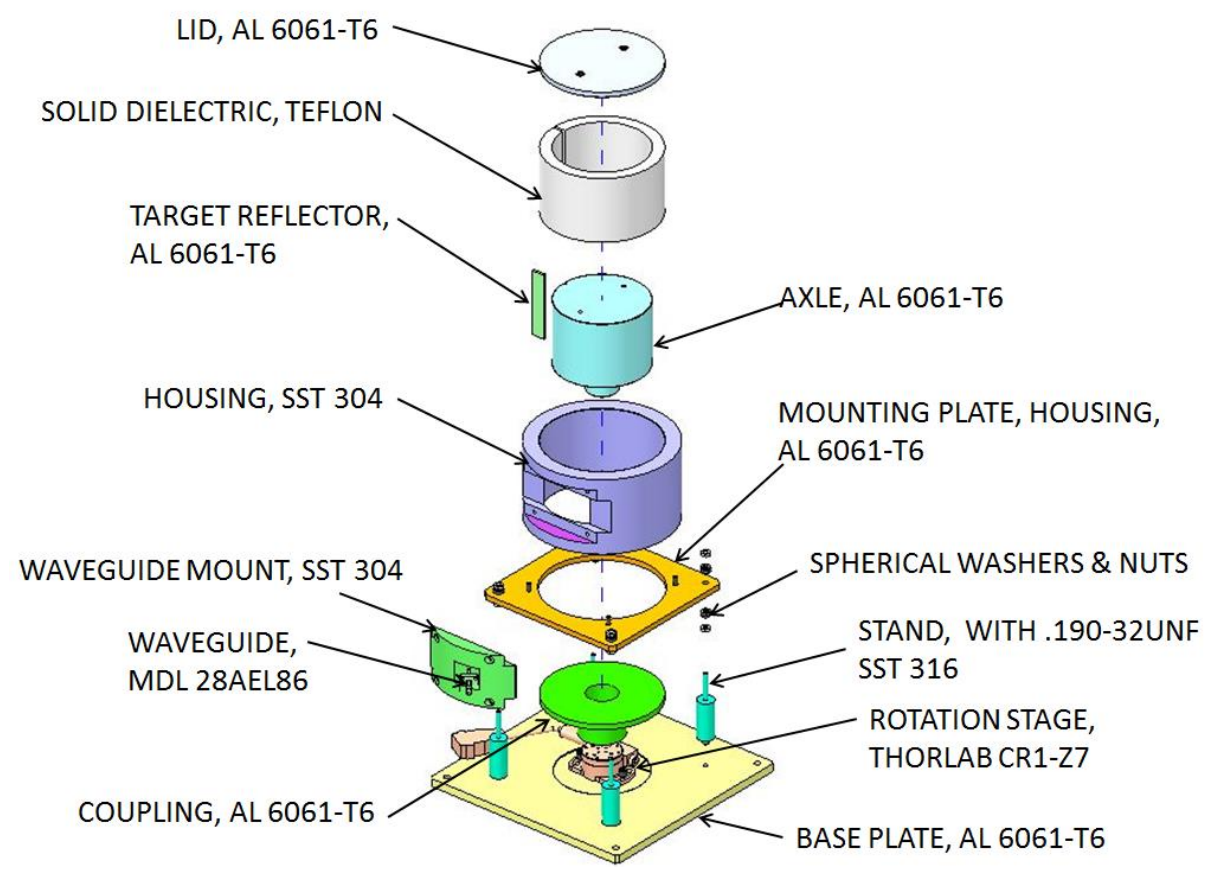

Figure 4.2: Expanded View of the RFMS. Not shown are additional waveguide mounts used. Critical components include the 'Solid Dielectric, Teflon', 'Target Reflector, $A L$ 6061-T6', 'Axle, $A L$ 6061-T6', and 'Housing, SST 304', which compose the circular geometry explored in this experiment [20].

If the RFMS is used for an explosive measurement, the Teflon ring would be replaced by an explosive. The purpose of the measurement is to be able to determine the location of the TR vs. time and thus the velocity of the TR. The phase of the return signal is measured and is used to calculate the position of the TR. The TR rotates around the fixture (simulating a detonation wave). The phase of the return signal will change twice as quickly as the change in TR location. So a half-wavelength change in distance of the TR will yield a full wavelength change in the S1l phase. Therefore, the ideal unwrapped phase will be perfectly linear, where every $2 \pi$ phase shift corresponds to a $\lambda / 2$ change in distance along the circumference of the TR. Since the fixture is encased in metal, all of the energy being transmitted into the fixture should return into the same port at some phase shift. Given the initial position of the TR and the changing return phase, the location of the TR can be determined.

LabVIEW code was written to automate the testing of the RFMS. '.NET' controls were used in LabVIEW to control the Thorlabs motor controller KDC101 and SCPI commands were used with the GPIB interface to control the VNA. Python code was also written and implemented to automate testing using GPIB. The tests performed were automated and taken using a Python script. 
The S11 measurements are dependent on frequency and mode structure within the fixture. Given the complex nature of the multi-modal structure, phase changes unpredictably, not in the ideal manner described above. Since there is a dielectric discontinuity between the Teflon ring and the air in the waveguide, some amount of energy would reflect off of the Teflon and go back into the waveguide. To try to measure more ideal phase results, we looked at two different ways to improve energy coupling into Teflon: Brewster angle variation and matching materials between dielectric and the material inside of the coax-waveguide adapter inside of the waveguide mount.

\subsection{Matching Improvements by Brewster Angle}

The waveguide mounts (hereafter referred to as "launchers") were manufactured at angles $60^{\circ}$ and $72^{\circ}$ (shown in Figure 4.3).

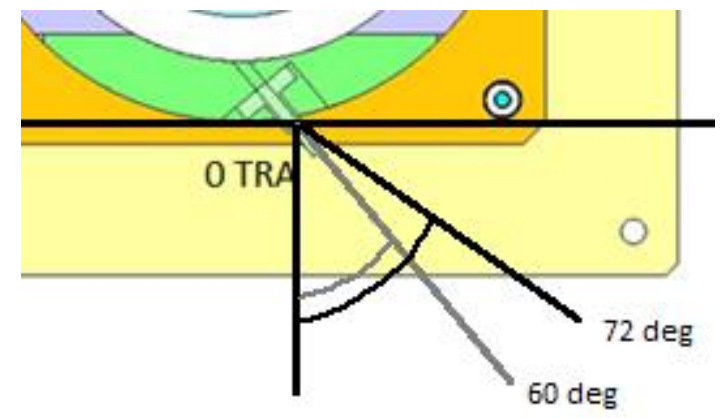

Figure 4.3: RFMS Launcher Angles. Manufactured at $60^{\circ}$ and $72^{\circ}$. Bottom of RFMS from Figure 4.1 shown.

The goal of the different mount angles was to vary the angle of incidence with relationship to the Brewster angle to see the effect on transmission. The Brewster angle, or the angle of total transmission, from one material into another with different dielectric constant is defined as Equation 4.1 [21]. Total transmission would result in no energy reflection at the air-Teflon boundary, improving the reliability of $\angle S 11$.

$$
\theta_{i}=\theta_{B}=\tan ^{-1} \sqrt{\frac{\epsilon_{2}}{\epsilon_{1}}} \quad \mu_{1}=\mu_{2}
$$

where the angle of incidence $\theta_{i}$ is normal to the surface of the boundary. Since Teflon $\varepsilon_{r}=2.2$ and air $\varepsilon_{r}=1$, using Equation 4.1, $\theta_{B}=56.0122^{\circ}$.

WR28 end-launch coax to waveguide adapters were used from $26 \mathrm{GHz}$ to $40 \mathrm{GHz}$ [22] in the launchers. 


\subsection{Matching Improvements by Material Matching}

This section will outline the equations used to describe the matching, then calculate the necessary values and show the matching improvement over an air-filled waveguide.

If the Teflon ( $\varepsilon_{r}=2.2$ ) cylinder is modeled as a rectangular waveguide with broad dimension 'a' (3.0000in.) and short dimension 'b' (1.0236 in.) [20], the cutoff frequency is Equation 4.2 [21]:

$$
f_{c, m n}=\frac{1}{2 \pi \sqrt{\mu \epsilon}} \sqrt{\left(\frac{m \pi}{a}\right)^{2}+\left(\frac{n \pi}{b}\right)^{2}}
$$

For the fundamental mode $\mathrm{TE}^{10}$, this reduces to Equation 4.3 .

$$
f_{c, 10}=\frac{1}{2 a \sqrt{\mu \epsilon}}=1.326 G H z
$$

Then the lower cutoff frequency of the cylinder should be approximately $1.326 \mathrm{GHz}$, which is well below the frequency range used in the measurements. This will result in the cavity being overmoded (when there are a large quantity of wave patterns inside of the waveguide from different higher-order modes), resulting in unpredictable phase (if one mode is present, the phase would change linearly; presence of higher-order modes changes the linear phase response in an unknown way).

After measurements were taken for both launchers, the launchers were filled with wax (estimated relative dielectric constant close to that of Teflon) to try to couple more energy into the Teflon dielectric by matching the two regions together. This would create a 3-material region as shown in Figure 4.4. Paraffin wax was used. Once the melted wax was poured into the launcher, a razor blade was used the remove excess wax to make the wax flush with the inner surface of the launcher. For small air gaps between the wax fill and the Teflon cylinder less than $1 \mathrm{~mm}$ (see Figure 4.6) the reflection coefficient improves over a pure air-Teflon transition for dielectric constants of 2-2.5 (see Figure 4.5).

Equations 4.4-4.8 are used to measure how much power is coupled into the fixture. These relations are defined in [21].

Equation 4.4 is the waveguide impedance, where $\eta_{\circ}$ is the impedance of free space, $f_{c}$ is the cutoff frequency of the waveguide, and $f$ is the frequency.

$$
\eta_{T E}=\frac{\eta_{o}}{\sqrt{1-\left(\frac{f_{c}}{f}\right)^{2}}}
$$

Equation 4.5 is the reflection between two regions, where $\eta_{i}$ is the waveguide impedance in region $i$. 


$$
\Gamma=\frac{\eta_{2}-\eta_{1}}{\eta_{2}+\eta_{1}}
$$

Equation 4.6 is the phase constant of an electromagnetic wave, or the change in phase per unit length traveled.

$$
\beta=\frac{2 \pi}{\lambda}
$$

Equation 4.7 is the input reflection into the three material region in Figure 4.4 assuming infinitely long Regions 1 and 3. The variable d is the width of Region 2.

$$
\Gamma_{i n}=\frac{\Gamma_{12}+\Gamma_{23} e^{-j 2 \beta_{2} d}}{1+\Gamma_{12} \Gamma_{23} e^{-j 2 \beta_{2} d}}
$$

Equation 4.8 is the percent of reflected power calculated from the input reflection in Equation 4.7.

$$
\% \text { reflected power }=|\Gamma|^{2}
$$

To measure the waveguide impedances to get accurate reflection calculations, the following numbers were used: standard WR28 has dimensions $a=7.11 \mathrm{~mm}$ and $\mathrm{b}=3.56 \mathrm{~mm}$. Table 4.1 lists waveguide impedances from Equation 4.4 in each material region.

Table 4.1: Waveguide Impedances Inside of the Structure, calculated from Equation 4.4. Used in Equations 4.5 and 4.7 to calculate input reflection into the Teflon.

\begin{tabular}{|l|c|c|c|c|}
\hline & $\begin{array}{l}\text { Cutoff } \\
\text { Frequency }\end{array}$ & $\begin{array}{l}\text { Impedance } \\
\text { at 15GHz } \\
(\Omega)\end{array}$ & $\begin{array}{l}\text { Impedance } \\
\text { at 26 } \\
(\Omega)\end{array}$ & $\begin{array}{l}\text { Impedance } \\
\text { at } \\
(\Omega)\end{array}$ \\
\hline $\begin{array}{l}\text { WR28 } \\
\text { Without } \\
\text { Wax }\end{array}$ & $\begin{array}{c}21.081 \mathrm{GHz} \\
{[21]}\end{array}$ & $\begin{array}{c}\text { Non- } \\
\text { propagating }\end{array}$ & 644.0 & 444.0 \\
\hline $\begin{array}{l}\text { WR28 With } \\
\text { Wax }\end{array}$ & $14.21 \mathrm{GHz}$ & 1179.0 & 450.0 & 403.0 \\
\hline $\begin{array}{l}\text { Teflon } \\
\text { Ring }\end{array}$ & $1.326 \mathrm{GHz}$ & 378.5 & 377.5 & 377.2 \\
\hline
\end{tabular}

Figure 4.4 is a horizontal cross-section of the geometry used in the RFMS if the circular structure is unwrapped onto one axis. The coax-waveguide transition used was an end-launch connector represented by the orange pin on the left of the figure. This pin is either immersed in air or wax. Region 2 is a gap of air, which accounts for the gap between the housing and the Teflon and a possible gap between the wax fill and the inner edge of the waveguide mount. An improvement in the input reflection would increase energy coupled into the Teflon region, which would indicated by a lower S1l than if Region 1 is filled with air. 


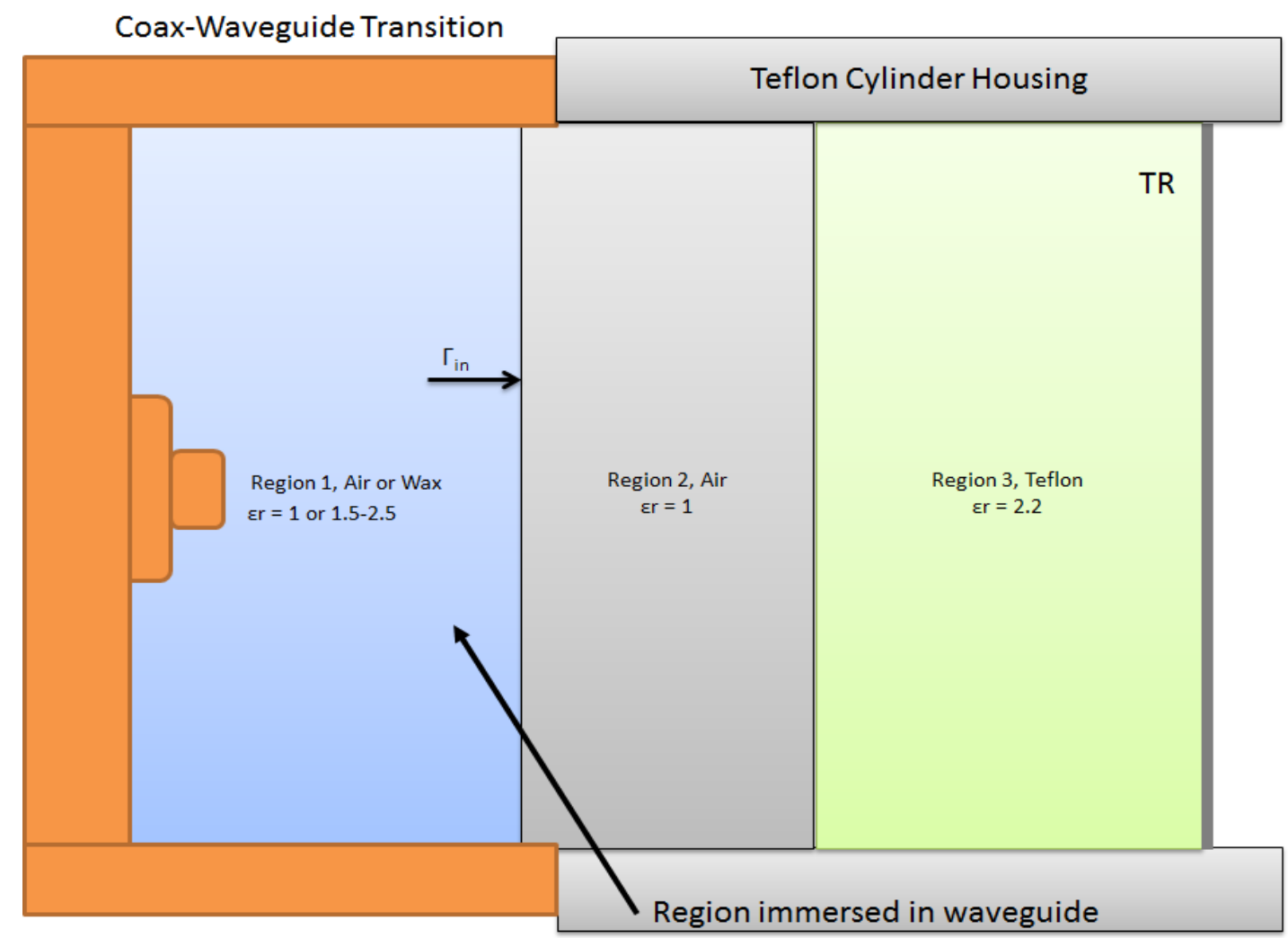

Figure 4.4: Three Material Reflection Diagram. Transitions from

wax into air, and into the Teflon ring. These transitions motivate filling the launchers with wax to couple more energy into the Teflon.

If Region 2 is smaller than $1 \mathrm{~mm}$ wide, then a dielectric constant of wax between 1 and 3 will optimize the results according to Equation 4.5. The dielectric constant of the wax was measured to see if it was close enough to Teflon to yield positive results. The dielectric constant was measured with a SPEAG DAK probe [23] between $5 \mathrm{GHz}$ and $50 \mathrm{GHz}$. After calibration, the wax was heated until melting, and then the probe was immersed in the wax. The dielectric constant of cooling, solidifying wax was measured, because liquid and solid wax have different dielectric properties. Measurements were recorded immediately after wax application, 19 minutes later, and 29 minutes later, shown in Figure 4.5. The dielectric constant ranges between 2.0 and 2.4 between $26 \mathrm{GHz}$ and $40 \mathrm{GHz}$. A final measurement was recorded after 15 hours. However, the measurement had decreased to approximately 1.5 and was inconsistent vs. frequency (not shown in Figure 4.5 for figure clarity). This is possibly due to wax peeling away from the probe as it solidifies. The wax region around the probe may be a heterogeneous air and wax mixture. 
The assumed dielectric constant value of 2.0 to 2.4 yields an input reflection between $-2 \mathrm{~dB}$ and $-10 \mathrm{~dB}$ lower than air from Figure 4.6, increasing energy coupling into the Teflon. Liquid wax was poured into the waveguide launchers, hardened, and then made flush with the inner edge of the waveguide mount for wax experiments.

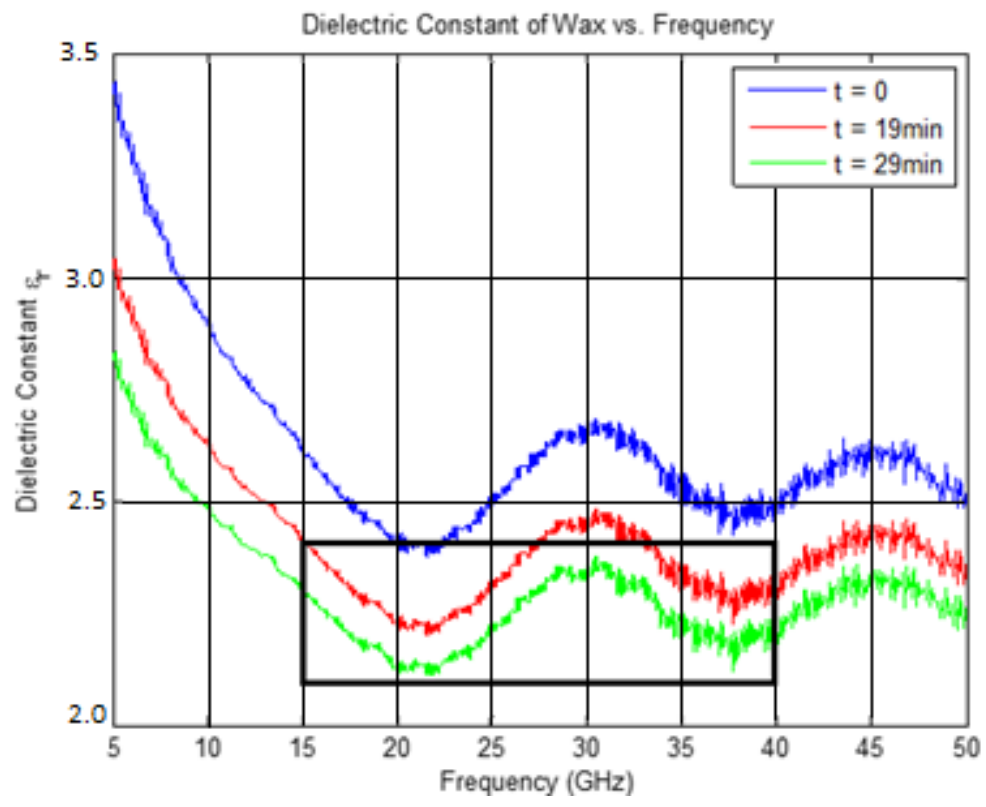

Figure 4.5: Solidifying Paraffin Wax Dielectric Measurements: Measurements made at 0 (blue), 19 (red), and 29 (green) minutes after application to probe region. The constant varies between 2.112 and 2.377 between $15 \mathrm{GHz}$ and $40 \mathrm{GHz}$. This constant is used

for Region 1 in Figure 4.4 to determine increase in energy coupling into the Teflon. 
Input Reflection Change vs. $\varepsilon_{\mathrm{r}}$ for Different Air Gaps

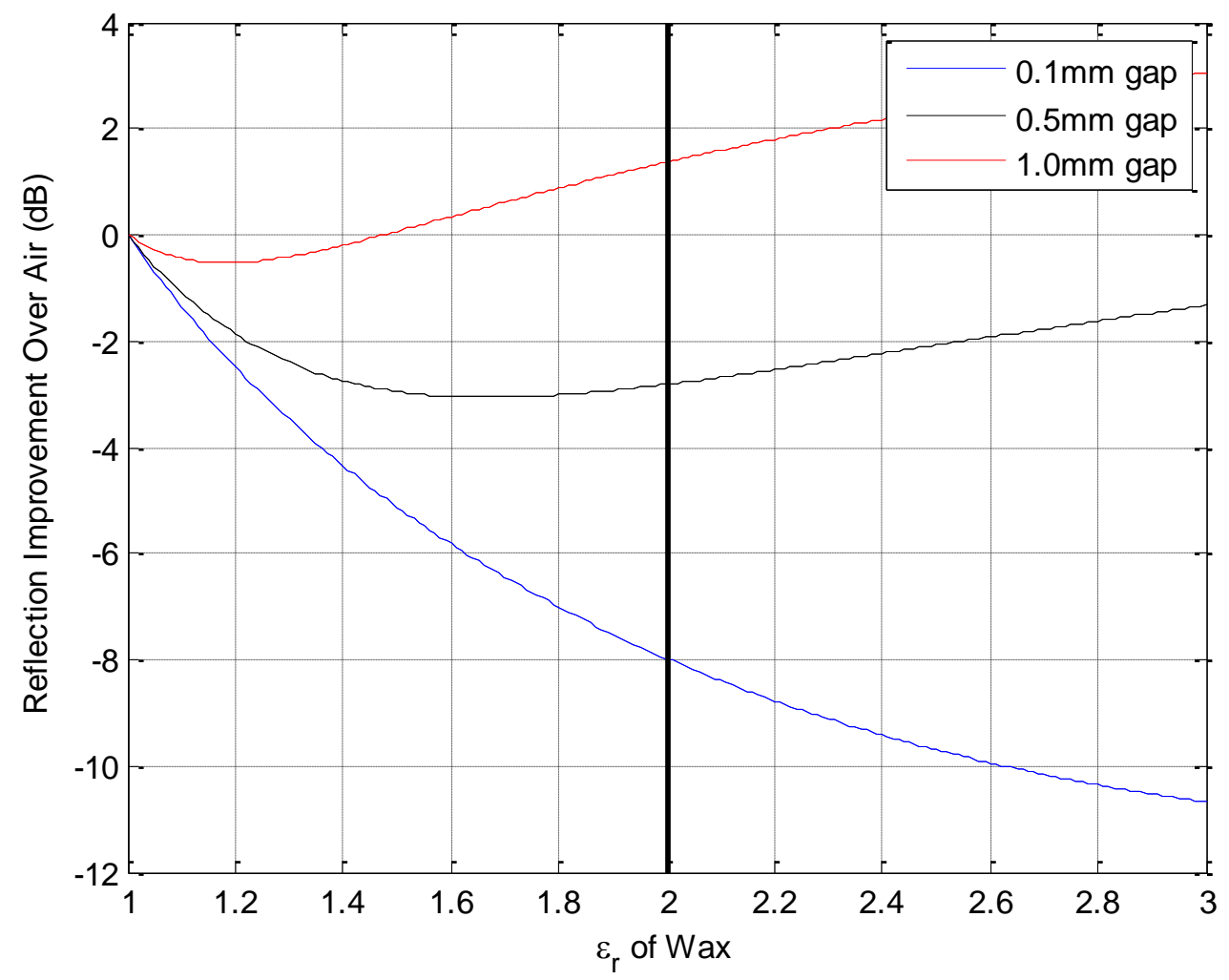

Figure 4.6: Wax-filled waveguide reflection improvement: 0.1 ,

$0.5,1.0 \mathrm{~mm}$ air gap. With worst case $\varepsilon_{r}$ of 1.5 , there is

improvement over no wax for Region 2 air gaps less than $1 \mathrm{~mm}$.

Two different measurements were taken for each of the launchers: S11 measurements were taken $0^{\circ}$ to $360^{\circ}$ TRA in increments of $0.25^{\circ}$ between $15 \mathrm{GHz}-40 \mathrm{GHz}$ without wax, and $26 \mathrm{GHz}-40 \mathrm{GHz}$ with wax (discrete TRA locations with a continuous frequency measurement). Continuous wave (CW) measurements between $15-50 \mathrm{GHz}$ with wax and $26 \mathrm{GHz}-40 \mathrm{GHz}$ in increments of $1 \mathrm{GHz}$ were taken as the target reflector rotated from $0^{\circ}$ to $360^{\circ}$ (continuous TRA measurement with discrete frequency measurements).

The individual phase measurements at each TRA from each S11 measurement for a single frequency were compiled to measure the changing phase at each frequency vs. the TRA (compiled results). Figure 4.7 shows the conversion from frequency swept S11 measurements into a compiled result. The CW measurement were run as a method to verify that this method of compiling individual phase points was accurate. Accuracy of the phase is shown in the section on Measurement Repeatability Testing. The CW case simulates an actual MI measurement, mimicking the moving detonation front with the moving TR. Measuring LS11 is critical because phase is used to extract detonation velocity. 


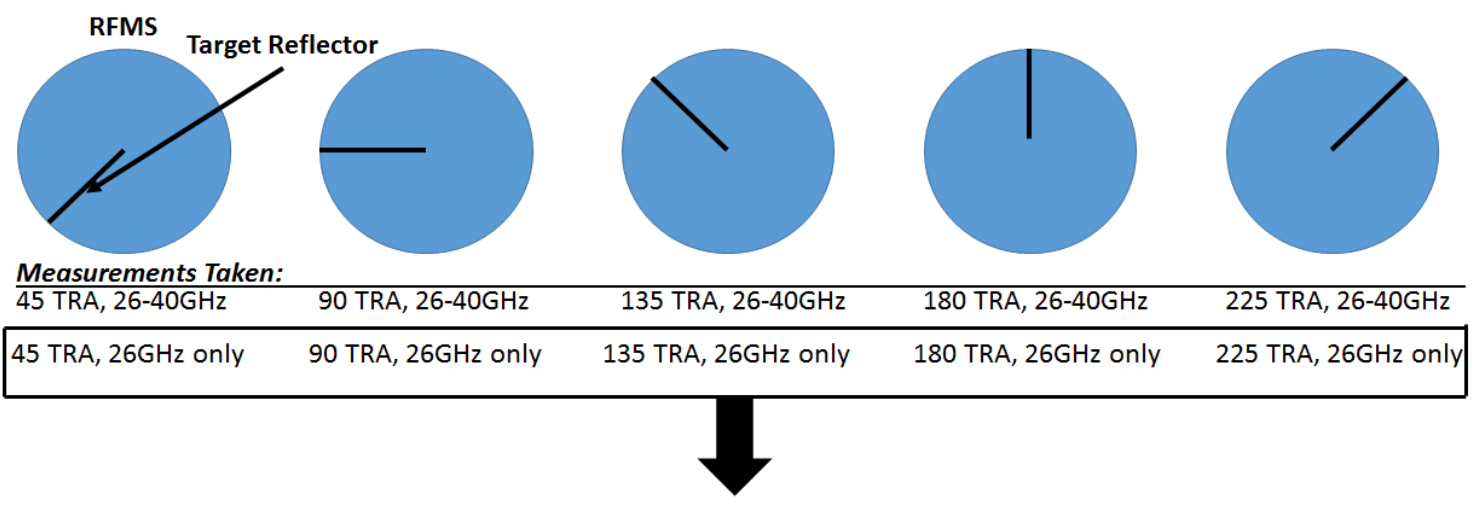

Phase of RFMS at 26GHZ = [... 45 TRA... 90 TRA... 135TRA... 180TRA... 225TRA...]

Figure 4.7: Compiled Results Example. Conversion from frequency swept phase at one TRA to a single frequency with swept TRA.

Four launcher configurations were tested: Launchers 1 and 2, with and without wax. Launcher 1 is $60^{\circ}$ and Launcher 2 is $72^{\circ}$.

\subsection{Recorded Measurements}

Table 4.2 displays RFMS tests with test ID index, launcher configuration, CW sweep or compiled results, and CW measurement rotation speed. 
Table 4.2: RFMS Tests.

\begin{tabular}{|c|c|c|c|}
\hline Test ID & $\begin{array}{l}\text { Launcher } \\
\text { Config. }\end{array}$ & Test Type & Additional Notes \\
\hline $\begin{array}{l}1 \\
2 \\
3 \\
4 \\
5 \\
6 \\
7 \\
8\end{array}$ & 1 & Compiled Results & $\begin{array}{l}\text { 0-360 taken over the } \\
\text { course of multiple } \\
\text { days, before } \\
\text { automation code was } \\
\text { written }\end{array}$ \\
\hline 13 & 1 & Compiled Results & $0-360^{\circ}$ \\
\hline 14 & 1 & Compiled Results & $0-89^{\circ}$ \\
\hline 15 & 1 & Compiled Results & $0-89^{\circ}$ \\
\hline 16 & 1 & Compiled Results & $0-360^{\circ}$ \\
\hline 17 & 1 & Compiled Results & $0-360^{\circ}$ \\
\hline 18 & 1 & Compiled Results & $0-360^{\circ}$ \\
\hline 19 & 1 & Compiled Results & $0-360^{\circ}$ \\
\hline 20 & 1 & CW Sweeps & $\begin{array}{l}26-40 \mathrm{GHz}, 1 \mathrm{GHz} \text { inc, } \\
5 \% \mathrm{~s}\end{array}$ \\
\hline 21 & 2 & Compiled Results & $0-360^{\circ}$ \\
\hline 22 & 2 & $\mathrm{CW}$ & $26-40 \mathrm{GHz}, 1^{\circ} / \mathrm{s}$ \\
\hline 23 & 2 & CW Sweeps & $26-40 \mathrm{GHz}, \quad 5^{\circ} / \mathrm{s}$ \\
\hline 25 & 1, wax & Compiled Results & $0-360^{\circ}$ \\
\hline 29 & 2 , wax & CW Sweeps & $5^{\circ} / \mathrm{s}$ \\
\hline 30 & 2, wax & CW Sweeps & $5 \% / s$ \\
\hline 31 & 2, wax & CW Sweeps & $1^{\circ} / \mathrm{s}$ \\
\hline 32 & 2, wax & Compiled Results & $0-360^{\circ}$ \\
\hline 33 & 1, wax & CW Sweeps & $5 \% / \mathrm{s}, 15-39 \mathrm{GHz}$ \\
\hline 34 & 1, wax & CW sweeps & $5^{\circ} / \mathrm{s}, 15-39 \mathrm{GHz}$ \\
\hline 35 & 1, wax & CW sweeps & $1^{\circ} / \mathrm{s}, 15-39 \mathrm{GHz}$ \\
\hline
\end{tabular}

The following results show example data from the RFMS. Since the RFMS is a novel structure that has not been measured before, these figures are important to show to indicate what data from an experiment will possibly yield in order to measure the detonation velocity of an explosive inside of the RFMS. Appendix B has more figures showing data compared from the four different launcher configurations.

Figure 4.8 shows |S11| in Launcher 1 without wax between $26 \mathrm{GHz}$ and $40 \mathrm{GHz}$. Ideally, $|\mathrm{S} 11|=0 \mathrm{~dB}$ since there should be no exit for the signal in the structure, assuming the metal casing is PEC. The large frequency-dependent changes in |S11| show that the behavior of the structure is not accurately captured by a simple waveguide model. 


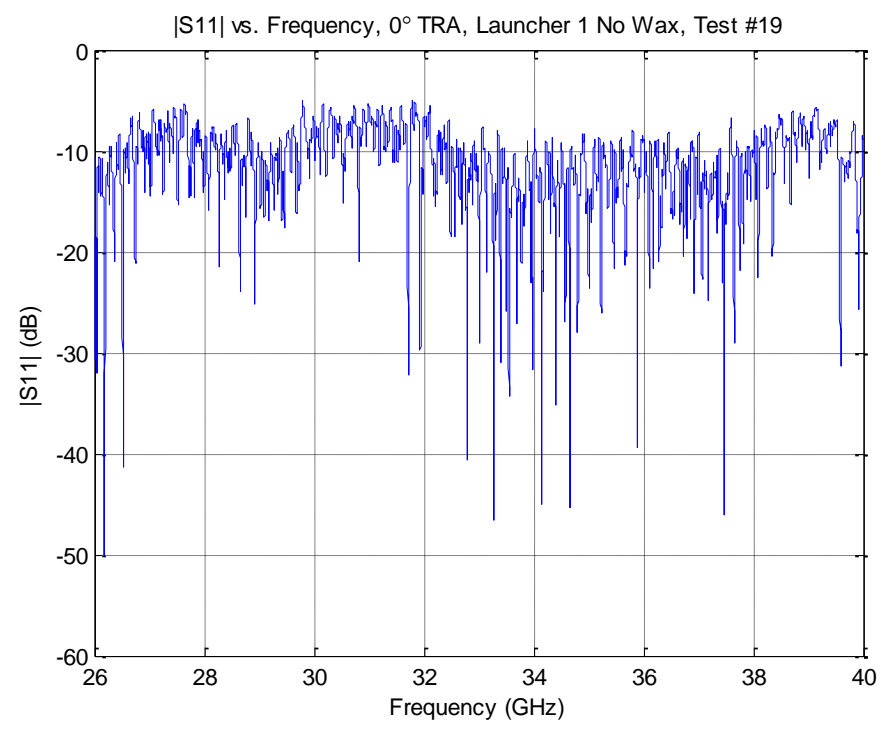

Figure 4.8: Input Reflection should ideally be $0 \mathrm{~dB}$, however, the magnitude varies greatly with frequency between $-5 \mathrm{~dB}$ and $-20 \mathrm{~dB}$, with some frequencies as low as $-50 \mathrm{~dB}$. From Test ID \#19, S11 over $26 \mathrm{GHz}-40 \mathrm{GHz}, 0^{\circ} \mathrm{TRA}$.

Figure 4.9 shows |S11| Vs. TRA at 26GHz. Similarly, the return should ideally be $0 \mathrm{~dB}$, but fluctuates widely throughout the rotation.

|S11| vs. TRA, 26GHz, Launcher 1 No Wax, Test \#19

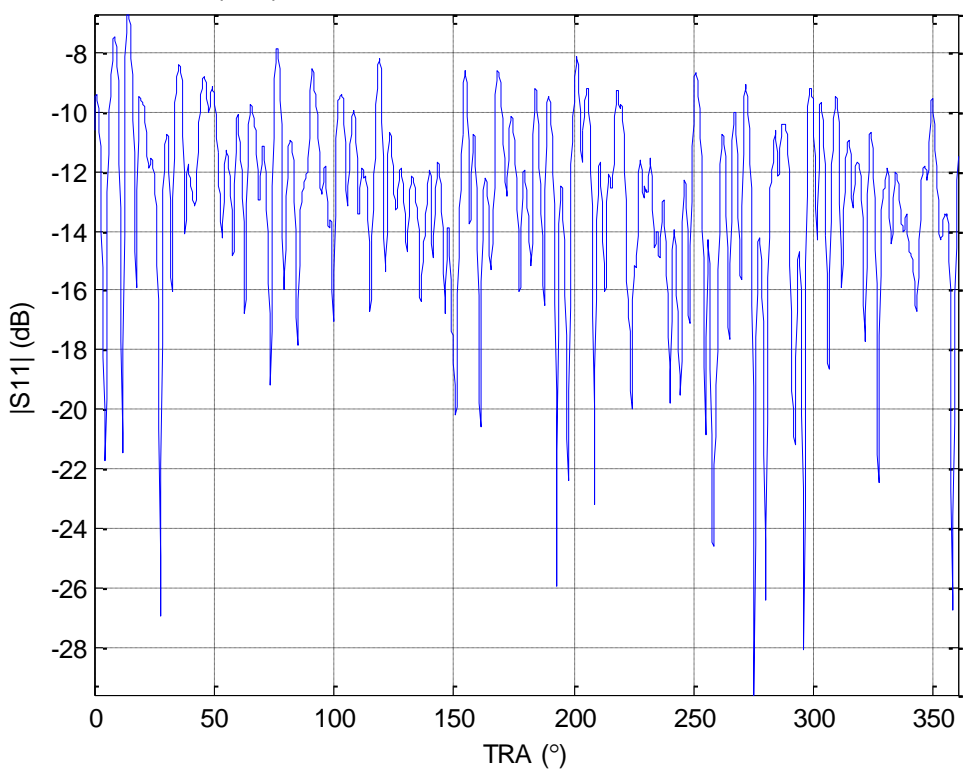

Figure 4.9: |S11| over one rotation of TRA at 26GHz. Input Reflection should ideally be $0 \mathrm{~dB}$, however, the magnitude varies largely with frequency between $-5 \mathrm{~dB}$ to $-20 \mathrm{~dB}$, with some points as low as $-30 \mathrm{~dB}$. From Test ID \#19. 


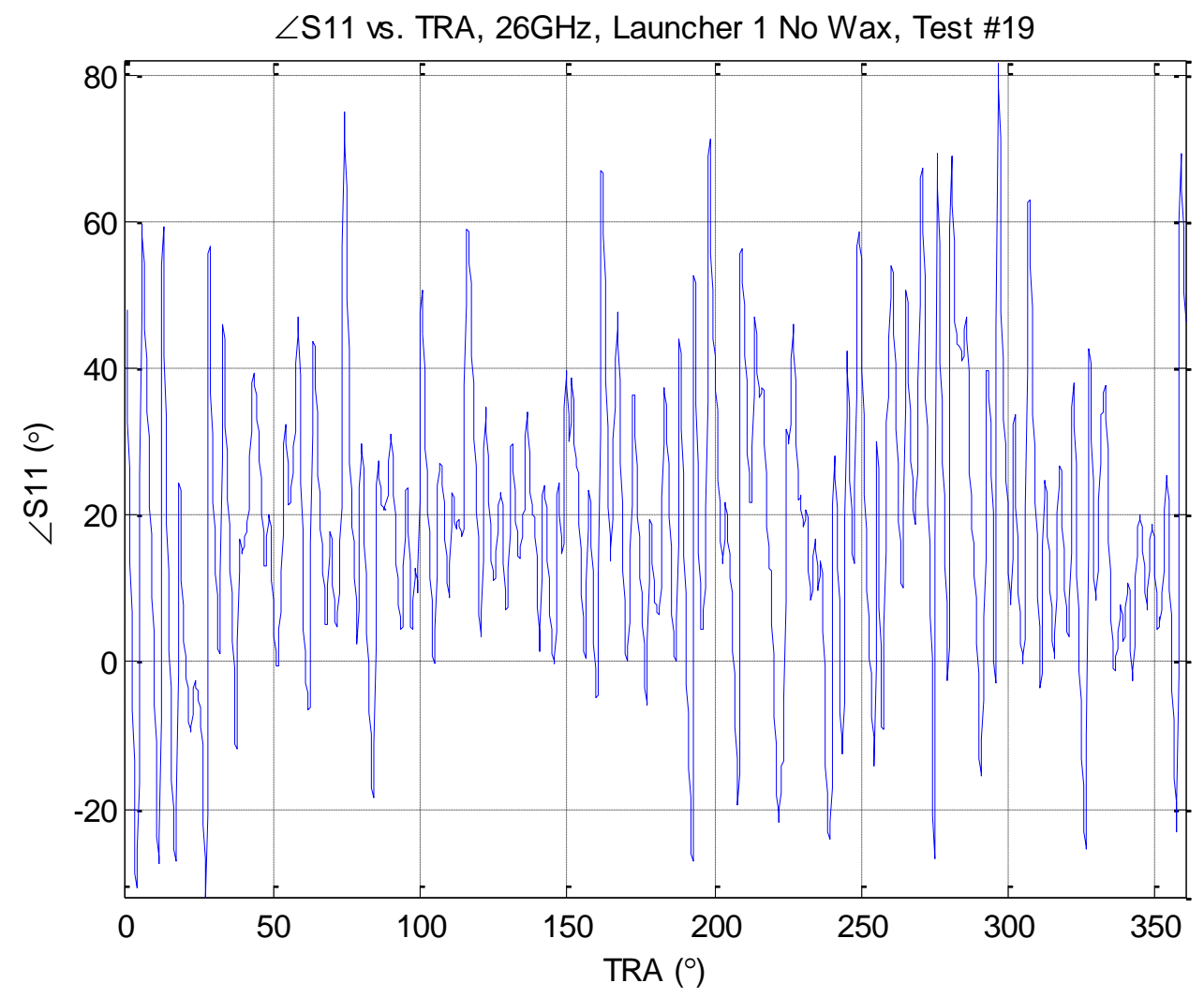

Figure 4.10: Input Reflection phase should ideally be a linear change VS. TRA, which would appear as a sawtooth waveform. Shown here, the phase changes rapidly and does not wrap, showing nonideality from the expected phase change. From Test ID \#19 LS11 of

Launcher 1 over one rotation of the TRA, no wax, at $26 \mathrm{GHz}$.

Figure 4.10 shows the phase of S11 vs. TRA. Ideally, this phase would constantly wrap like a sawtooth, similar to the top right of Figure 4.18. The complexity of the structure results in a highly non-ideal phase return at some frequencies.

\subsubsection{Measurement Repeatability Testing}

Since we are comparing data from different test configurations, repeatability between different measurements is necessary. Figure 4.11 shows initial and repeated $\angle S 11$ vs. TRA completed within one hour of each another. This shows relaxation of the SMA connectors during the time the test is running has a small, but negligible effect on the results on the phase. 


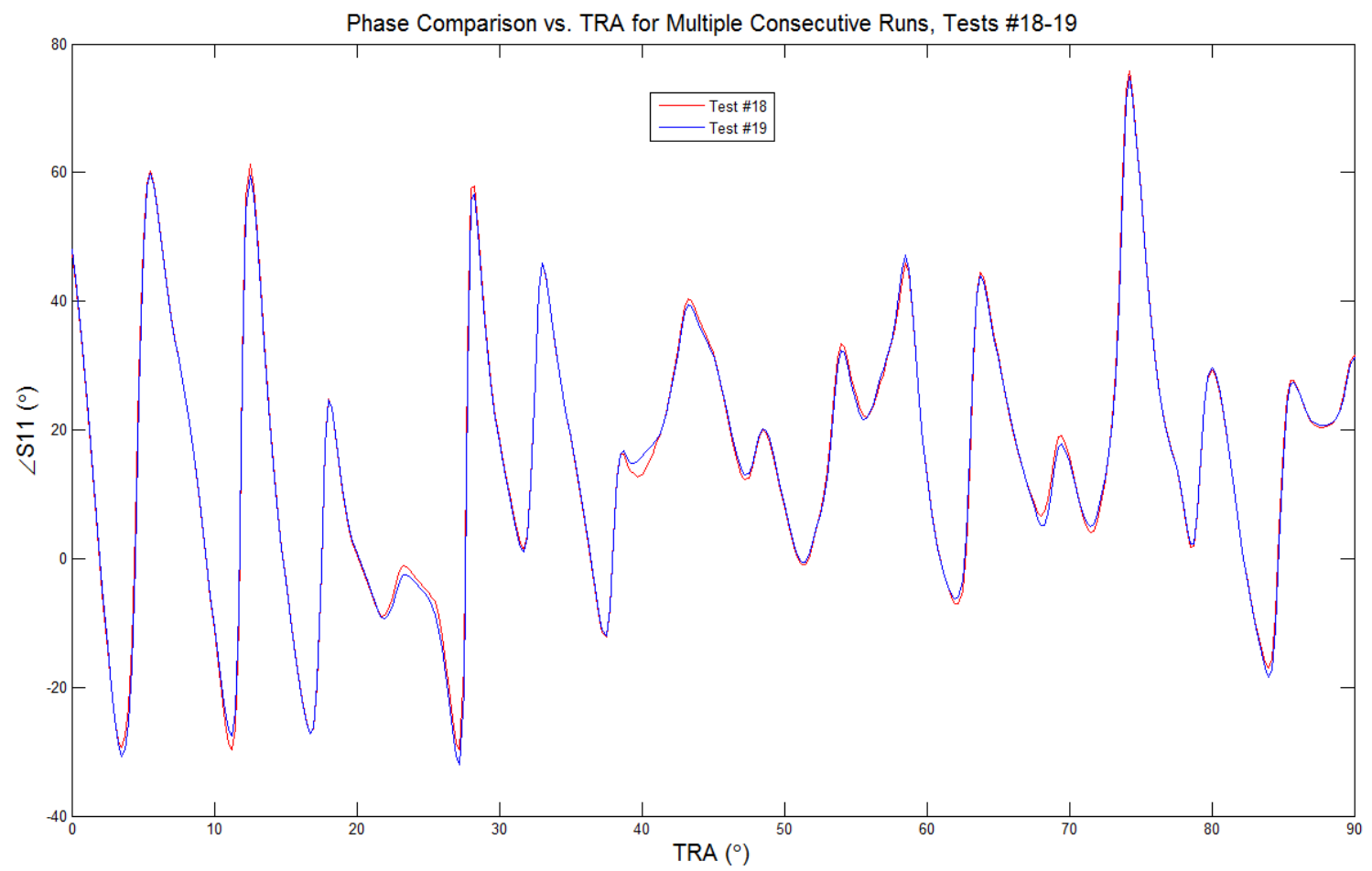

Figure 4.11: Comparison in phase between consecutive runs for

Tests \#18 and \#19 at $26 \mathrm{GHz}$ The strong similarity in phase measurements indicate repeatability between phase measurements, allowing for some time of connector relaxation.

Two measurement types are recorded: CW measurements and compiled results. CW measurements simulate actual phase data from an explosion measurement. Since CW measurements take longer and result in more wear on the structure, compiled results were desired. Figure 4.12 compares a CW measurement and compiled results from the same launcher configuration. To ensure that phase data is taken at the start of the motor sweeping and the stop of the motor, there was a slight delay before and after the measurements, resulting in a problem scaling CW phase to compiled phase perfectly. This results in the phase offset seen in Figure 4.13. However, the phase between the two different sets of results is subjectively identical so that the compiled phase results are acceptable to use instead of CW measurements. 


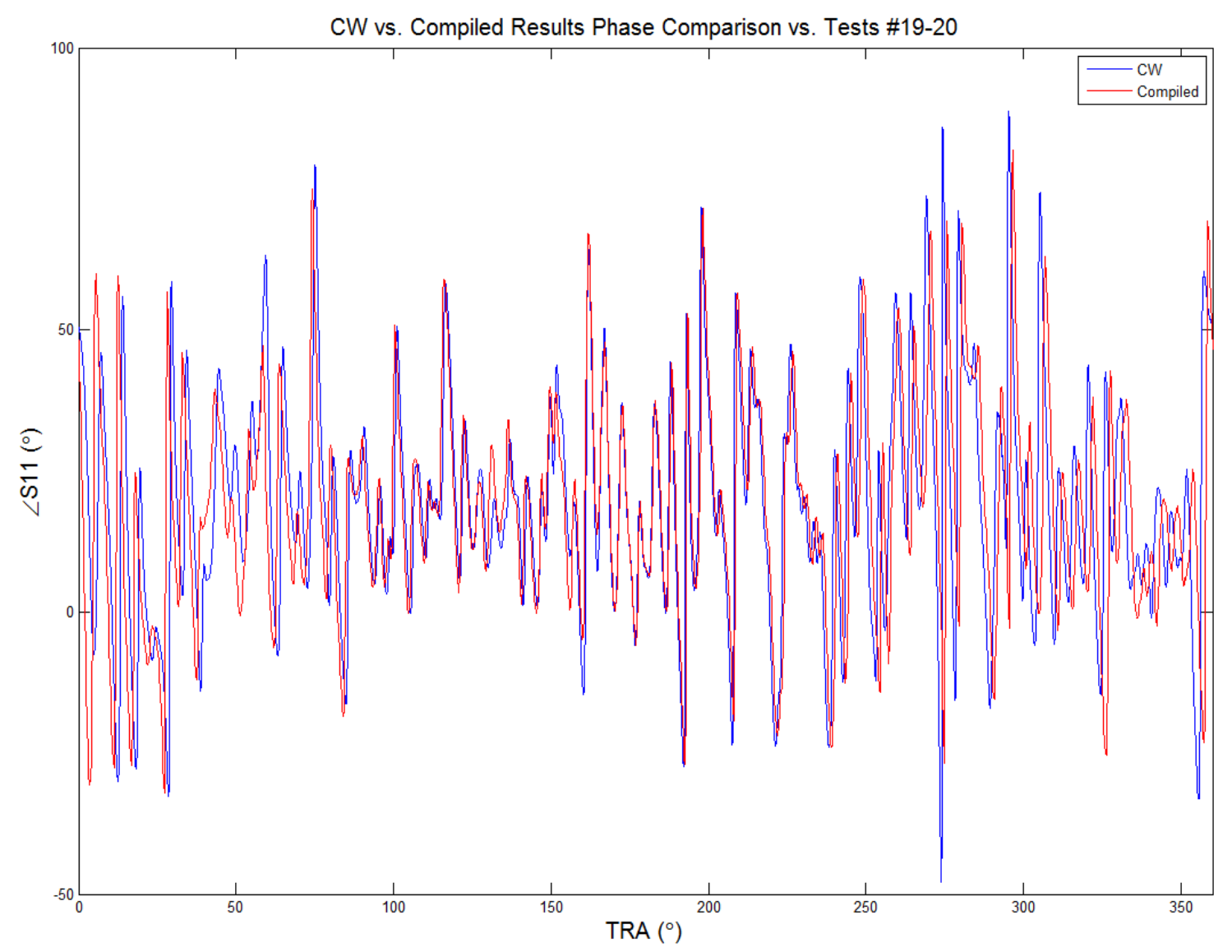

Figure 4.12: Phase Vs. TRA for compiled results and CW, which are subjectively identical indicate that the method of compiled phase points yields identical results to a CW measurement. CW sweep phase vs compiled results phase at $26 \mathrm{GHz}$ in Launcher 1, no wax for Tests \#19 and \#20.

\subsubsection{Results Repeatability Testing}

Another method to test the repeatability between different tests is comparing the final results from each test. One of the metrics used to measure a good frequency is $R^{2}$, which will be discussed in the Analysis of Input Reflection section. Four different tests were taken using the configuration of Launcher 1 with no wax. These are Tests \#16-19. Figure 4.13 shows the $\%$ difference in $R^{2}$ in Tests \#17-19 and Test \#16. Between any two tests, 75\% of the frequencies differ by less than $1 \%$. This indicates that the final results are acceptably repeatable for this structure. 


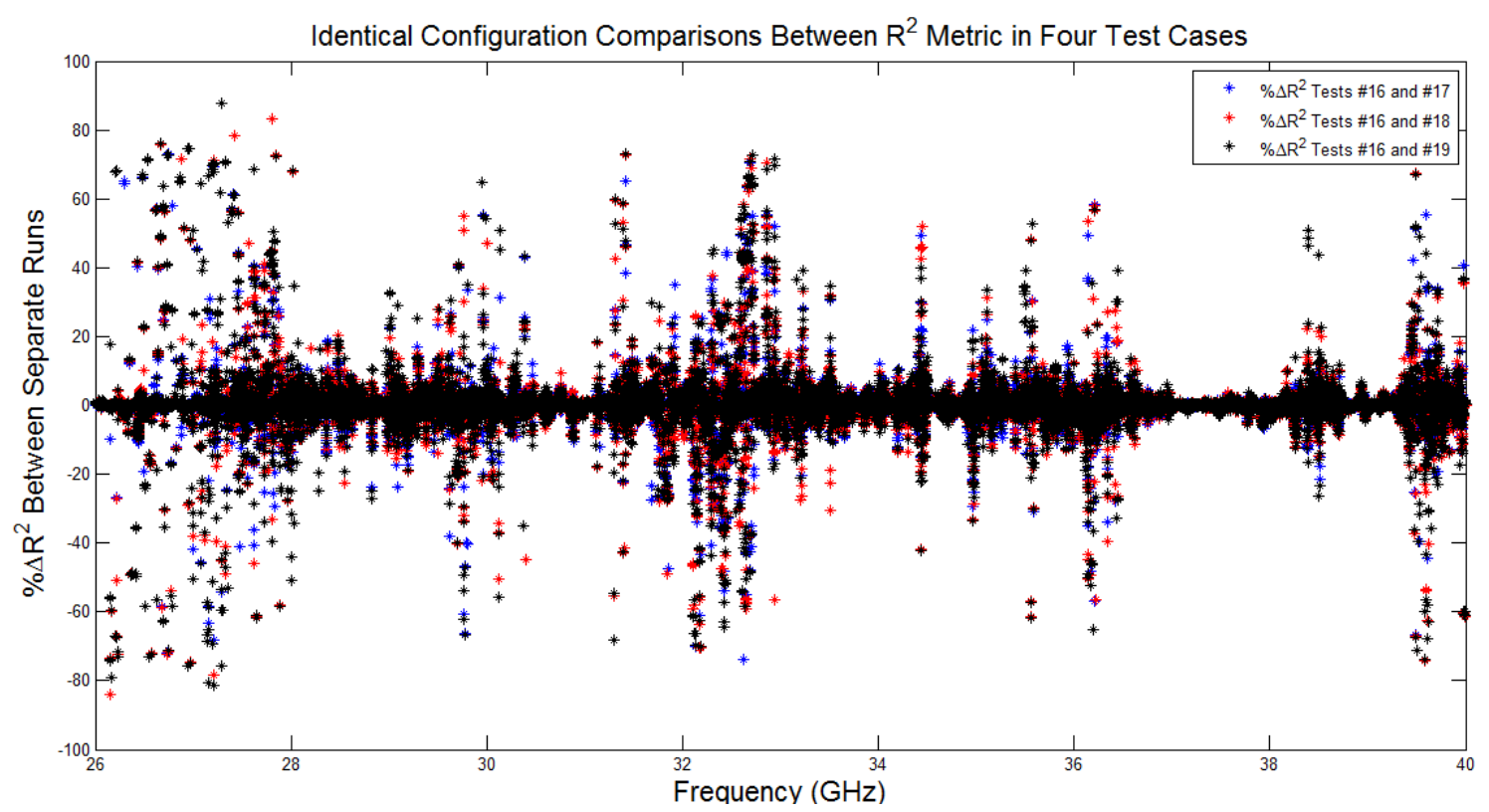

Figure 4.13: Identical Configuration Comparisons Between $\mathrm{R}^{2}$ Metric in Four Cases, Tests \# 16, 17, 18 and 19. The o difference in $\mathrm{R}^{2}$ between any two tests is less than 1\% for $75 \%$ of the frequencies. This indicates an acceptable amount of repeatability in testing.

\subsubsection{Energy Coupling Improvement Testing}

To see if the wax increased the amount of energy coupled into the RFMS, we compared the variance and mean of |S11| over TRA for all frequencies. Figure 4.14 shows |S11| variance between $26 \mathrm{GHz}$ and $28 \mathrm{GHz}$ for Launcher 1 with and without wax. The variance changes between frequencies, and is not consistently lower or higher in one configuration over the other. Launcher 2 had similar inconsistent variance changes.

|S11| variance and mean over all TRA were compared between Launchers 1 and 2 without wax to see if changing launcher angle increased energy coupled into the RFMS. Figure 4.15 shows Launcher 1 and Launcher 2 variance. 


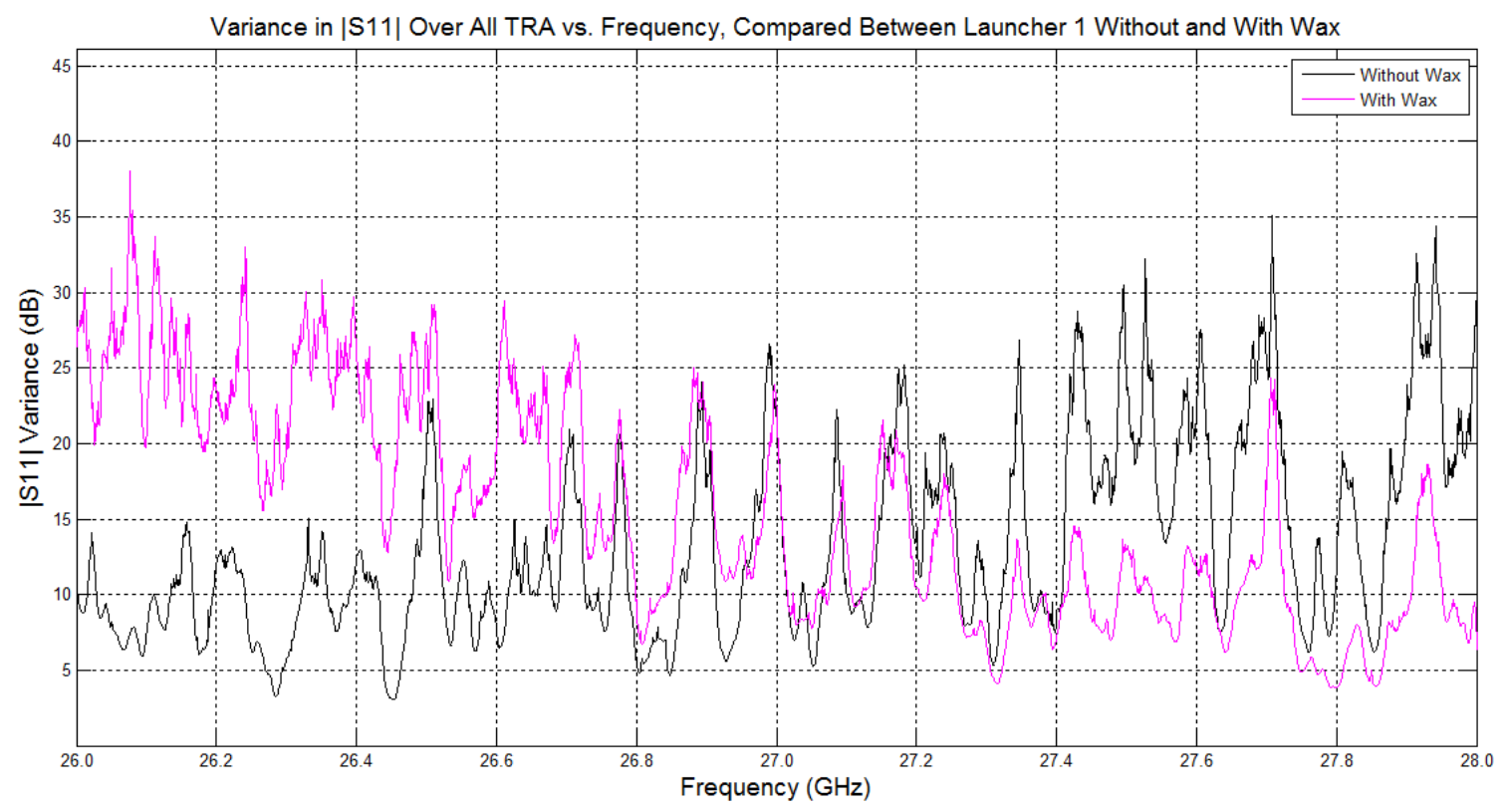

Figure 4.14: Variance in S11 magnitude vs. frequency between $26 \mathrm{GHz}$ and $28 \mathrm{GHz}$ between the waveguide filled with wax and without wax from Tests \# 19 and 25. Variance in the magnitude increased

at some frequencies and decreased at some frequencies in the overlapping frequency range between the two configurations. The effect of filling the launchers with wax is hard to determine if it helped overall.

Figure 4.16 compares average $|\mathrm{S} 11|$. Between Launcher 1 and Launcher 2 there is no clear decrease or increase in magnitude over all frequencies. However, between the configurations with and without wax, the overall |S11| increased in the configurations with wax, shown by the cyan and black traces above the red and blue traces as high as about $15 \mathrm{~dB}$. This indicates that filling the waveguide launchers with wax was unsuccessful.

Figure 4.17 shows the average S11 magnitude taken over all TRA for each frequency vs. the $\mathrm{R}^{2}$ value for that frequency (discussed in the next section). The correlation between the two values indicates that frequencies with better $R^{2}$ have lower average |S11|. Therefore, the higher overall |S11| with the wax-filled launchers in Figure 4.16 indicates that the launchers filled with wax decreased the performance of the system. Lower |S11| for better phase performance is possibly due to more energy being coupled into the fixture, which would result in more energy being absorbed in the fixture. Table 4.3 shows the correlation coefficients for the four launcher configurations. 
Table 4.3: Correlation coefficients between average $|\mathrm{S} 11|$ and $\mathrm{R}^{2}$.

\begin{tabular}{|l|c|c|c|c|}
\hline Test \# & 19 & 21 & 25 & 32 \\
\hline $\begin{array}{l}\text { Correlation, } \\
\rho\end{array}$ & -0.8109 & -0.7356 & -0.8063 & -0.7937 \\
\hline
\end{tabular}

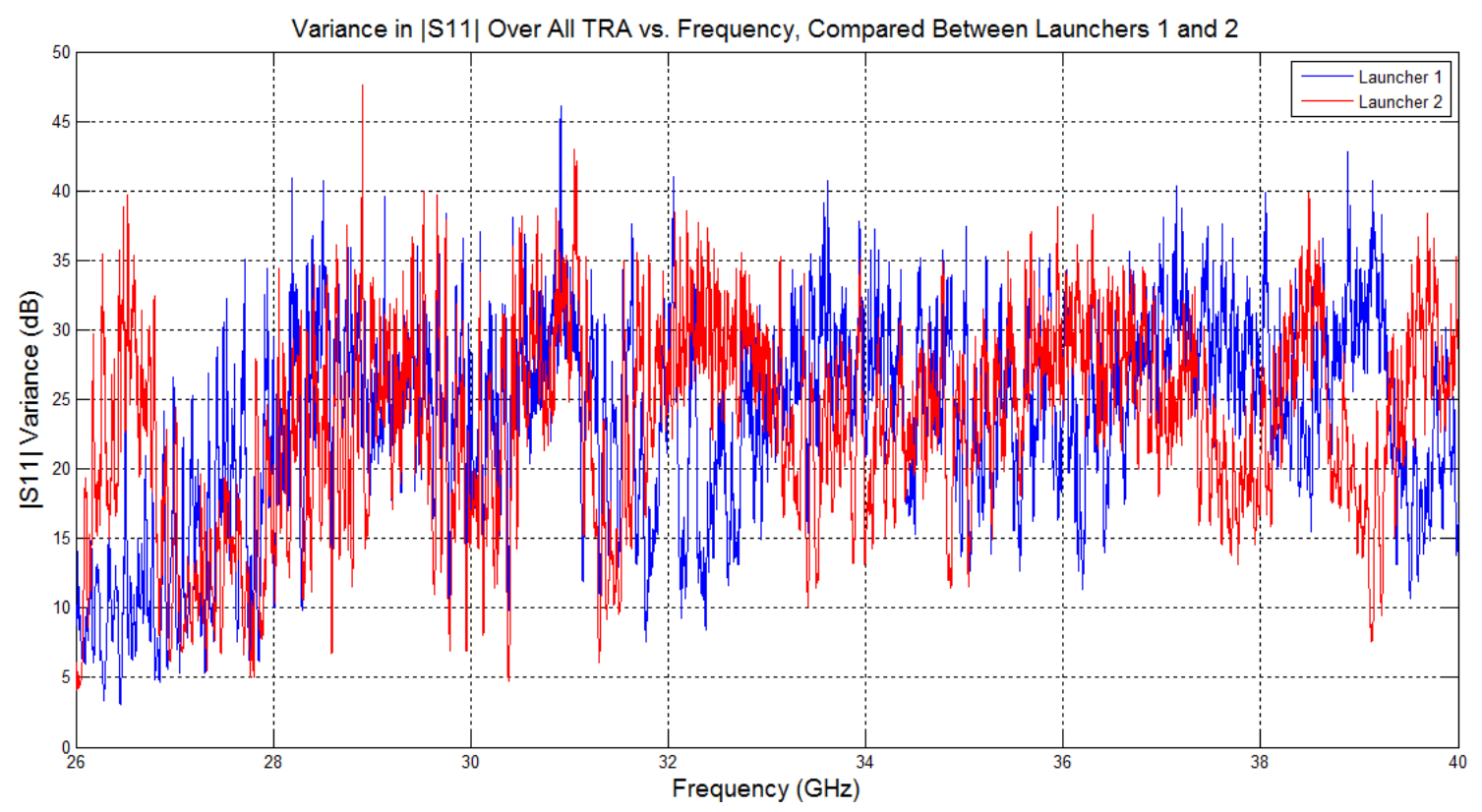

Figure 4.15: Variance in |S11| between Launchers 1 and 2 without wax. There is no constant increase or decrease in variance over the range $26 \mathrm{GHz}$ and $40 \mathrm{GHz}$, so it is hard to tell if the launch angle variation helps the measurement at all. 


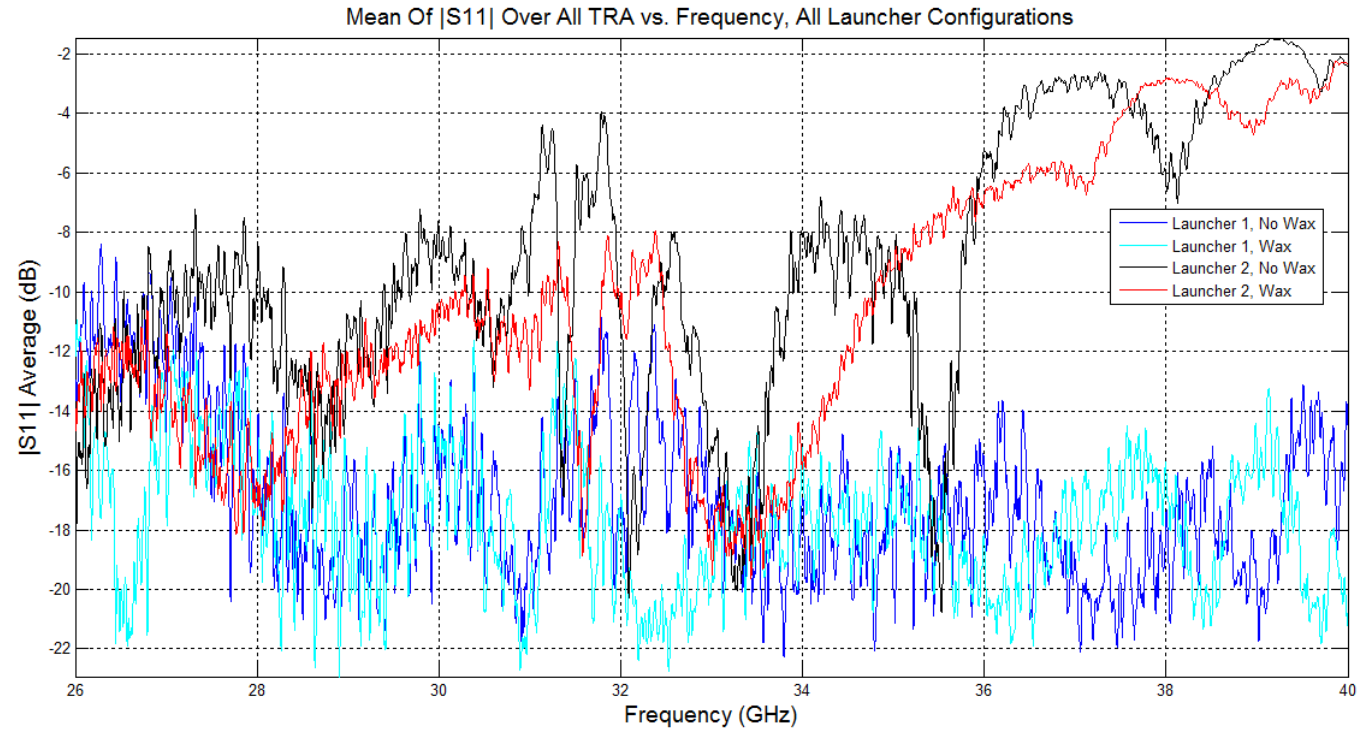

Figure 4.16: |S11| mean from all launchers 26-40GHz. Launchers with wax had higher $|S 11|$, indicating more energy coupled into the fixture.

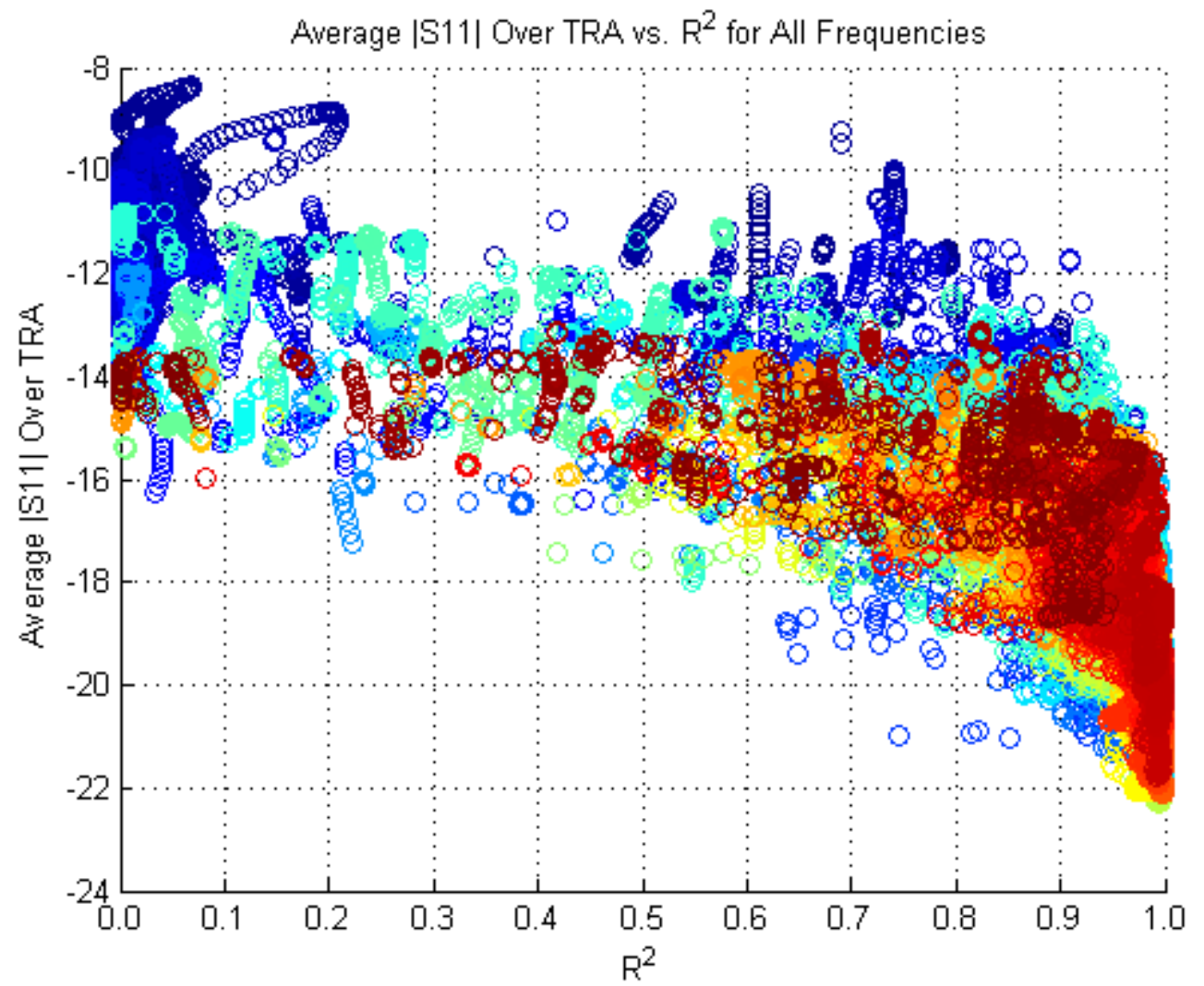

Figure 4.17: Average |S11| taken over all TRA for each frequency vs. $R^{2}$. Correlation coefficient between average $|S 11|$ and $R^{2}$ is $p$ $=-0.8109$, indicating that signals with better linear phase have lower |S11| values. 


\subsection{Analysis of Input Reflection}

Two methods were used to determine usable frequencies for the RFMS where the TRA would be easily predictable by the return phase. Being able to determine TRA location based on S1l phase is the most important result from this analysis because that is how detonation velocity is calculated.

The first method is to take the phase from S11 for compiled results of each frequency and unwrap it. A linear fit is applied to the unwrapped phase and an $\mathrm{R}^{2}$ metric is used to determine how well the unwrapped phase follows the fitted line. An $R^{2}$ value of 1 represents a perfectly linear phase and is the ideal case.

It is important to note that the MATLAB code used to unwrap the phase for $R^{2}$ is sensitive to a user-determined threshold. Since phase measurements are discrete, the phase between 2 adjacent points will not always be $\pi$. For the analysis performed, a phase different threshold for the code to unwrap the phase of 1.5 radians was used. Different results may occur if a different threshold is used, or if more points are taken in the experiment to get a more continuous phase measurement.

The second method addresses the non-ideality and somewhat random behavior of phase for each frequency and correlates actual phase zero-crossings with expected phase zero-crossings based on wavelength. Every $\Pi$ shift in the TRA should cause a full wavelength of phase change in the phase (as discussed previously). If the phase is wrapped, then the phase should cross through zero every $\lambda / 4$ due to the additional zero crossing because of the wrapping. Based on the wavelength of the signal, the expected zero crossings are computed for how often they should occur based on the change in TRA, and are then correlated with the actual computed crossings to give a best-fit to take into account changes in starting phase. If an actual zero crossing is within $\lambda / 8$ of the expected zero crossing, it is counted. The two outputs from this method are the number of points matched, and the average match error, which is the average error between the actual zero crossing and the location of the expected crossing. Here I define the zero-crossing metric to show how periodic the signal is, where a value of 1 shows a signal that is periodic across the time record and 0 shows that there is no apparent periodicity in the signal in Equation 3.4. This metric disregards the average match error.

$$
\text { Д }=\frac{\# \text { points recognized }}{\# \text { points expected }}
$$

Both analysis methods measure how periodic the phase is in some way. An ideal phase response unwraps perfectly and is periodic in a triangular shape (see example phase in the upper right of Figure 4.18). The $R^{2}$ method looks at linearity of the unwrapped 
phase. However, the zero-crossing method only looks at expected zero crossings and periodicity. For an ideal signal the zerocrossing method will yield a good result. The zero crossing method is meant for signals that do not unwrap well, considering the nonidealities of the phase return from the overmoded structure.

Figure 4.18 shows an example analysis of the zero crossing method for clarity.

The bottom left of Figure 4.18 shows the correlation between expected crossings and the actual crossings. In this ideal example, the number of actual crossings and expected crossings is identical, and the expected crossings have been shifted to the left to test the correlation at different starting phase points. This array of expected points is shifted from $\lambda / 4$ before the first actual zero to $\lambda / 4$ after the first zero to test the best starting phase point. The expected results from this example analysis are $100 \%$ Д with an average of $0^{\circ}$ error. The actual results from this example analysis are $100 \%$ म with an average $0.3667^{\circ}$ error (difference between actual and expected crossings) on each point.

The correlation between actual zero crossings and expected zero crossings accounts for extra crossings (as is the case with a noisy signal such as in the bottom right of Figure 4.18 by only associating with the best possible point that yields the lowest error for any particular expected zero. The results from the example noisy signal analysis is $100 \%$ With an average $0.2249^{\circ}$ error per point. 

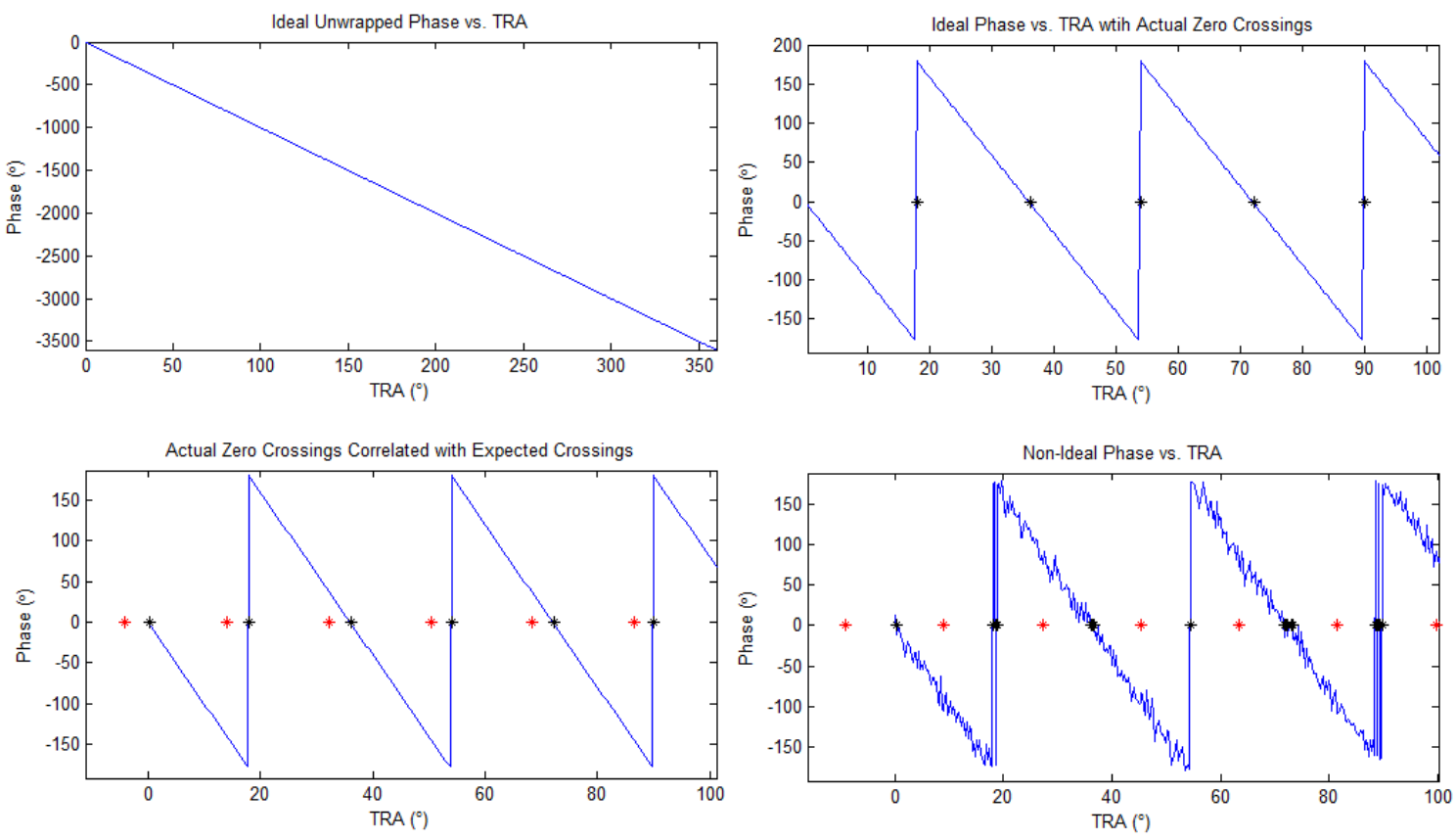

Figure 4.18: Top left: ideal unwrapped phase, over $360^{\circ}$ TRA, unwrapped from $0^{\circ}$ to $-360^{\circ}$; top right: ideal phase from top left, wrapped to between $-180^{\circ}$ and $180^{\circ}$, black dots shown are the detected zero-crossings; bottom left: ideal wrapped phase, black dots shown are the actual crossings, red markers shown are the expected crossings in the process of being correlated with the actual crossings (the markers are not fully correlated with the actual crossings in this figure, and will yield a lower errorper-point if shifted to the right) bottom right: ideal wrapped phase with white noise added (to model overmoded nature and nonideality in actual phase return). If the signal is noisy, extra zero-crossings will appear. However, the method deals with this issue by only correlating the closest zero-crossing with the expected crossing.

One possible drawback of the $Д$ metric is that it cannot account for phase variations so rapid that the algorithm associates an actual zero crossing with an expected zero crossing, but only because there a lot of noise in the signal. This noise would lead to a falsely increased Д metric.

$\mathrm{R}^{2}$ metrics were used to test all four launcher configurations. The I method was first computed for the launchers filled with wax since the launchers with wax were expected to have higher performance. The results did not come out as expected, so only $\mathrm{R}^{2}$ analysis was performed on the launchers without wax. Sample results are shown in Figures 4.19-23, and more complete results of my analysis are shown in Appendix C. Figure 4.19 shows the $\mathrm{R}^{2}$ for Launcher 1 without wax. There is a lot of variation with frequency, but there are a few bands of frequencies that have overall higher $\mathrm{R}^{2}$. Figure 4.20 
shows the Д metric shown for Launcher 1 without wax. There is less variation in the results over $\mathrm{R}^{2}$ and less banding.

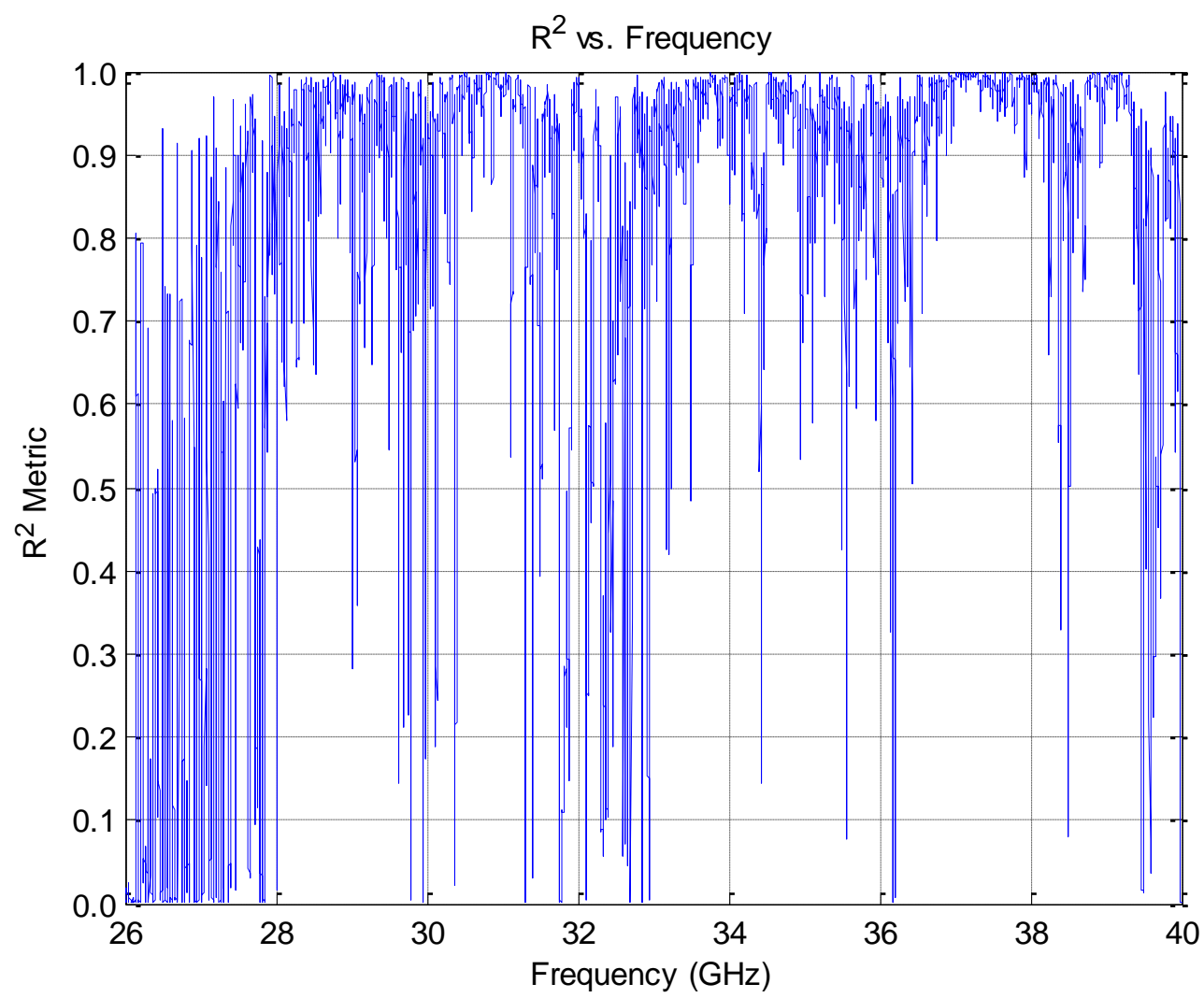

Figure 4.19: $\mathrm{R}^{2}$ : a linearity metric of 1 indicates ideal phase, and a linearity metric of 0 indicates no phase unwrapping. Frequency bands between $\sim 33 \mathrm{GHz}-35 \mathrm{GHz}$ and $37 \mathrm{GHz}-38 \mathrm{GHz}$ have higher overall phase linearity and may be suitable for tests. Test \#19. 


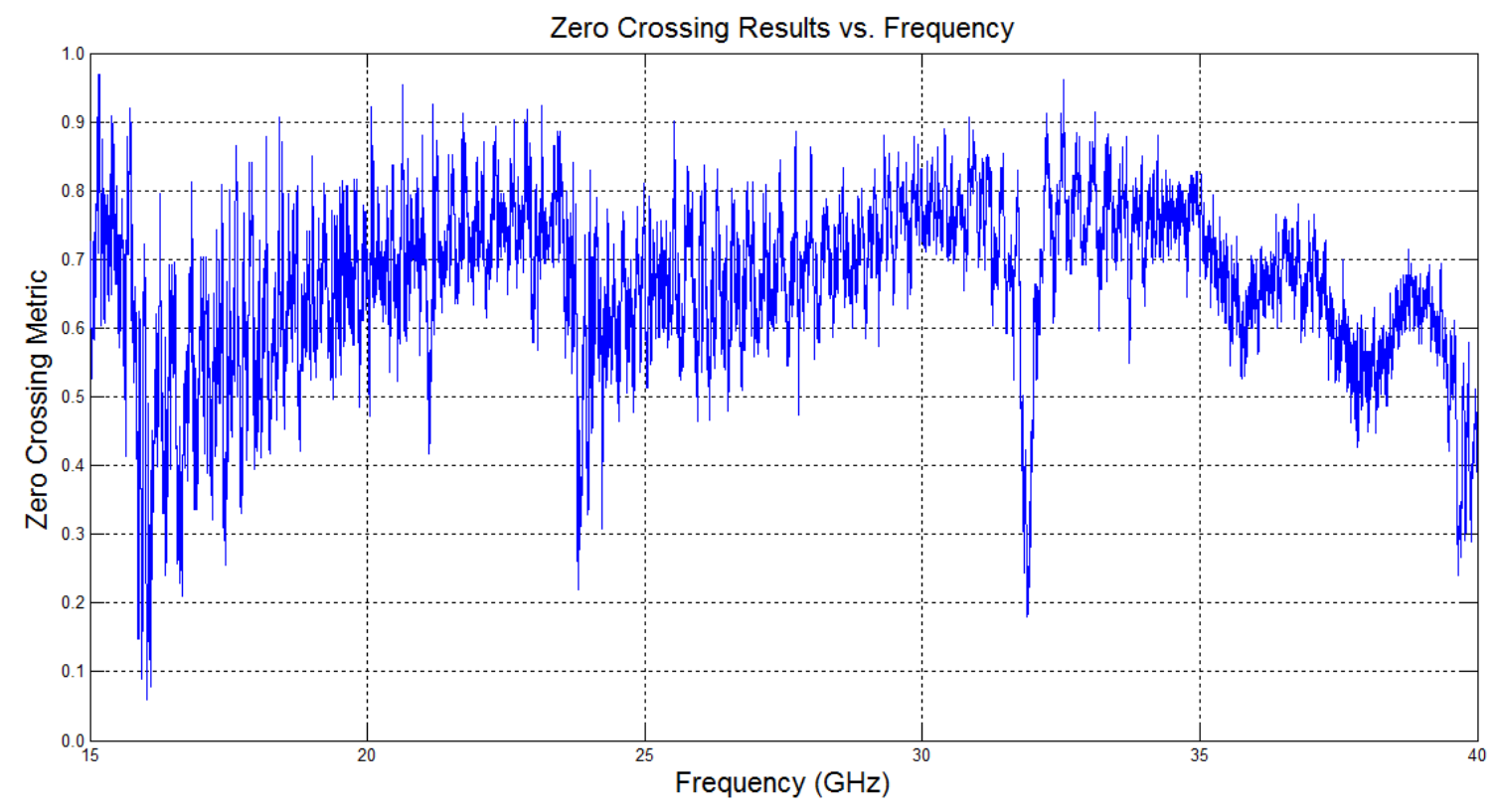

Figure 4.20: Results of zero-crossings tests. Д indicates how frequently the phase crossed zero to when it should, indicated periodicity in the phase. The results do not vary as much as the $\mathrm{R}^{2}$ method. For Test \#25.

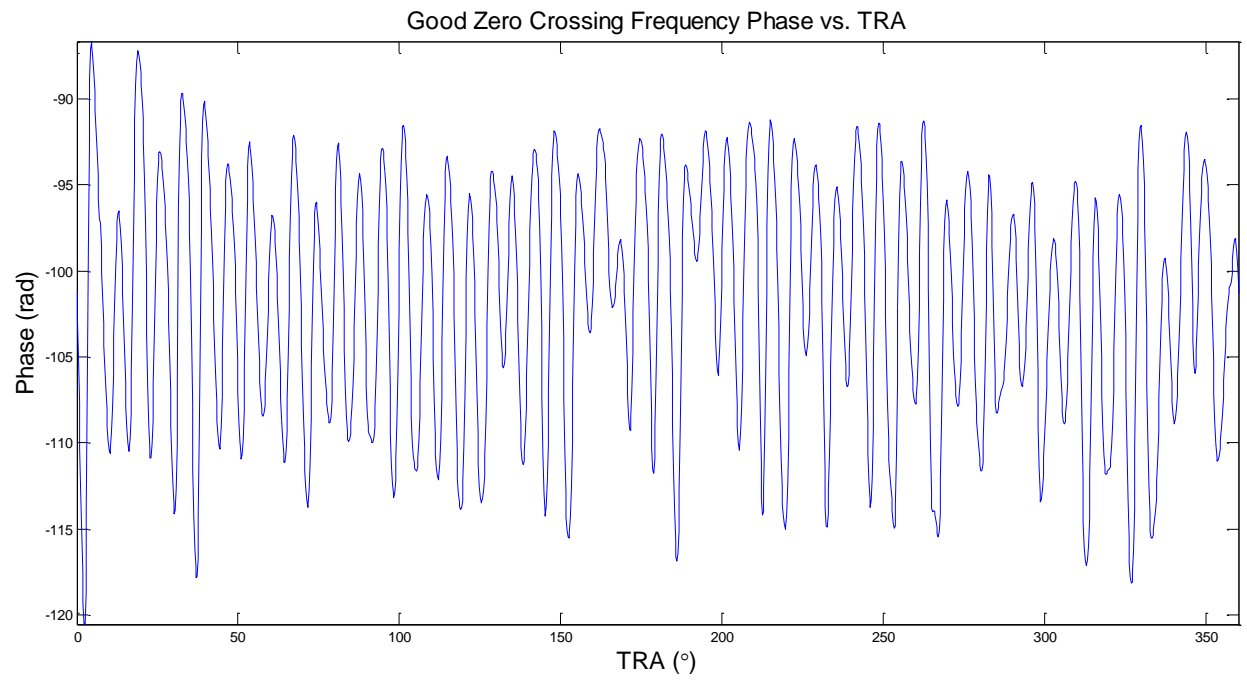

Figure 4.21: The best frequency indicated in Figure 4.20 actual phase. A high value from the zero-crossings method does not necessarily indicate linear phase unwrapping, but indicates periodic nature within the phase. At $15.167 \mathrm{GHz}$.

Figure 4.21 shows the point from Figure 4.20 with the highest Д value. Although the metric was high, there is almost no phase wrapping in the signal ( $R^{2}$ is 0.0064 for comparison), but there does seem to be a very consistent change in phase vs. TRA. 
Figure 4.22 shows the phase from the point in Figure 4.19 with the highest $R^{2}$ value. There is a large amount of consistent phase wrapping in this signal.

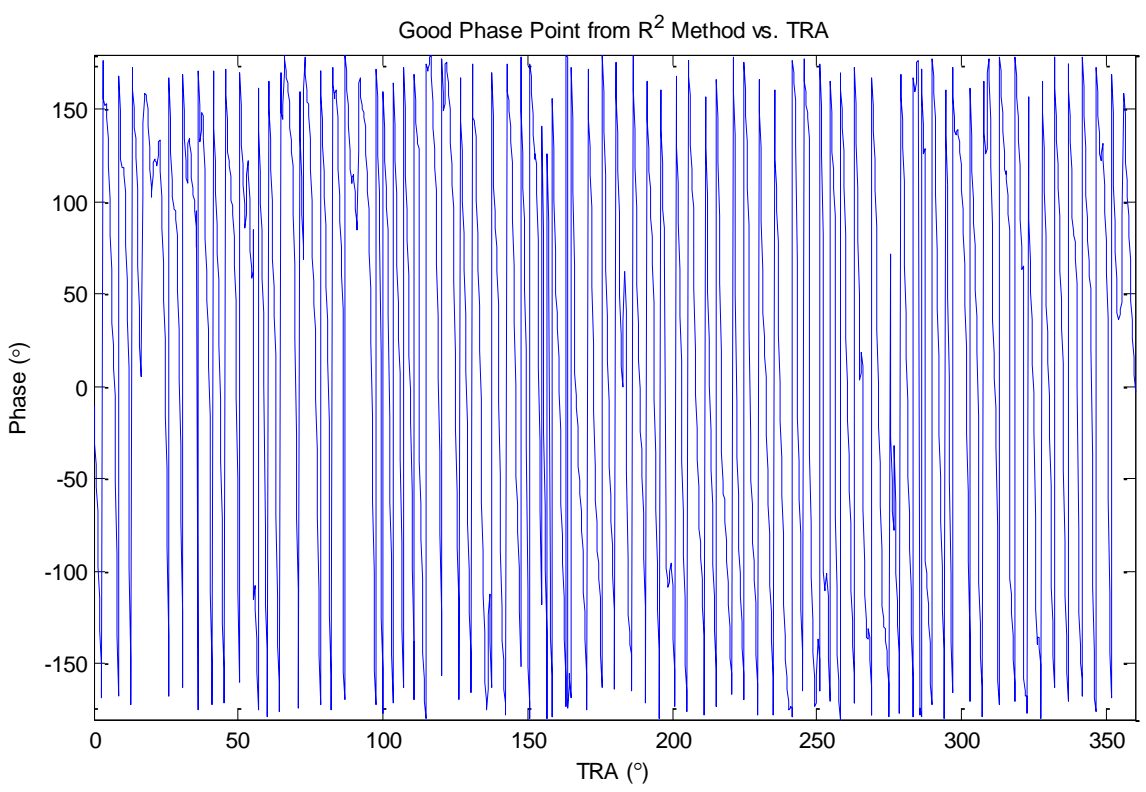

Figure 4.22: Wrapped phase example of a good point from Figure 4.19, at $39.1978 \mathrm{GHz}$. The $\mathrm{R}^{2}$ method metric indicates a high amount of phase linearity.

Figure 4.23 (left) shows the unwrapped phase from Figure 4.22. This phase is almost perfectly linear. To determine the threshold for what a good $\mathrm{R}^{2}$ value is, phases were looked at with lower $\mathrm{R}^{2}$ values. Figure 4.23 (right) right shows a frequency with a $R^{2}$ of .9152 . The phase w did not unwrap linearly. The threshold of .995 was chosen for good frequencies, which yielded about 5\% of good frequencies for Launcher 1 without wax. The threshold for Д was chosen to be .85, which yielded a similar amount of good frequencies as the $\mathrm{R}^{2}$ method. 

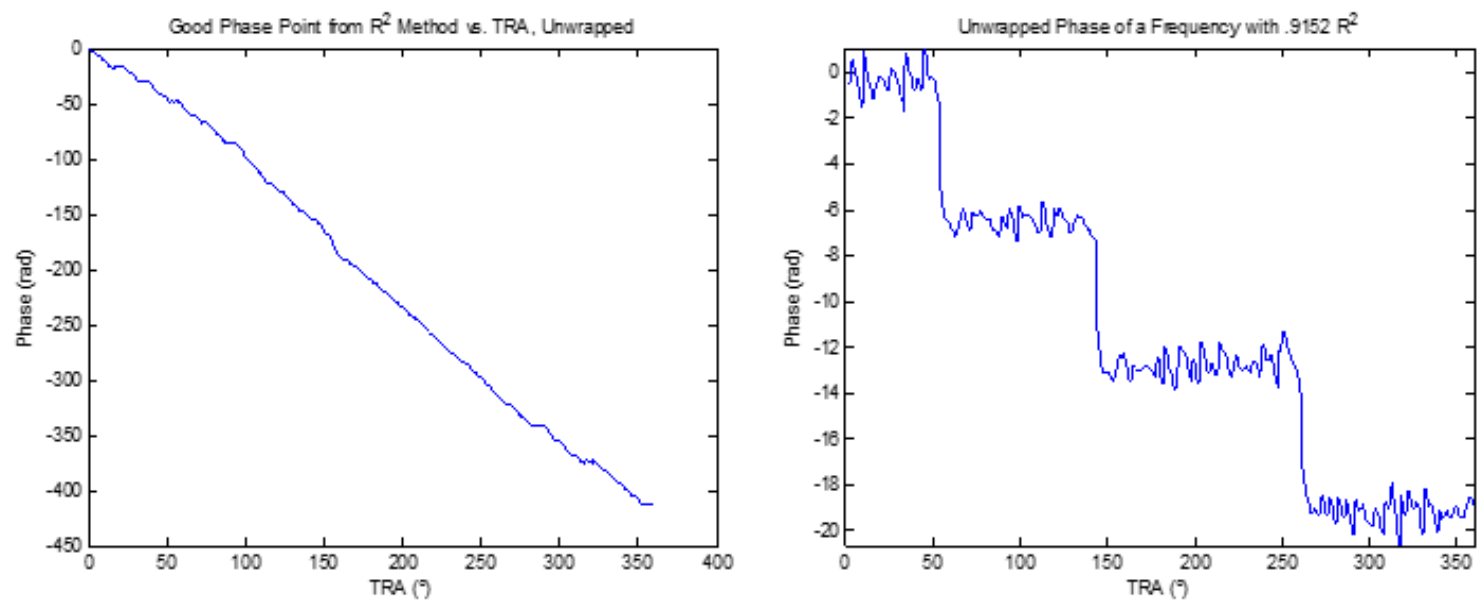

Figure 4.23: Left: unwrapped phase example of a good frequency, 39.1978GHz, from Test \#19. Right: phase of a frequency with larger $R^{2}$ metric of 0.9152 when unwrapped has a jagged and irregular pattern. Therefore the threshold for the of good frequencies was increased to 0.995 for $\mathrm{R}^{2}$.

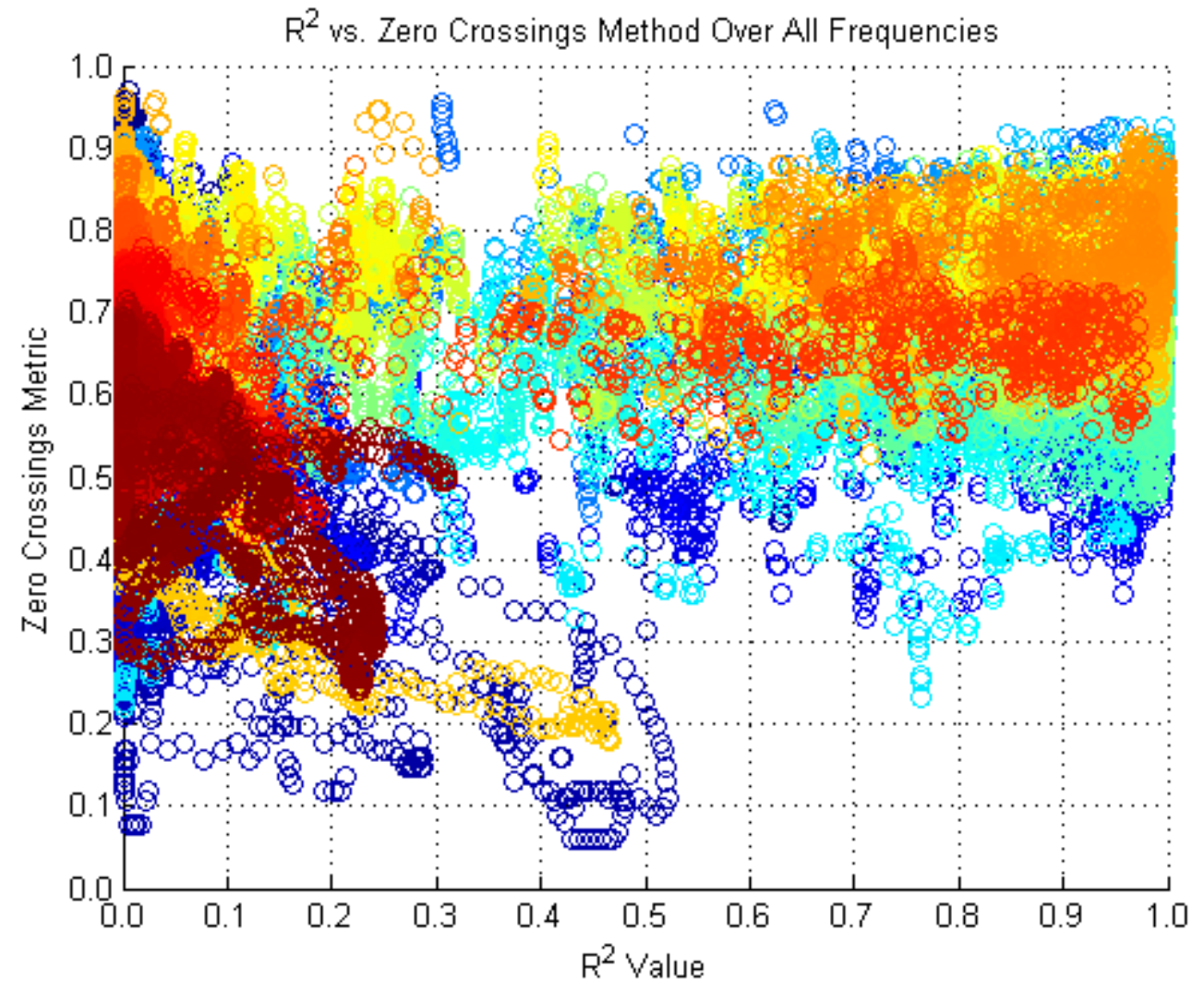

Figure 4.24: $R^{2}$ metric compared to Д metric; low frequencies indicated by dark blue and higher frequencies are indicated by dark red, Test \#25. 
To compare the performance of the two metrics in relationship to one another, $R^{2}$ and $Д$ were plotted against one another in Figure 4.24. Ideally the metrics would yield a straight line through the origin, indicating that a good frequency in one metric is a good frequency in the other. Correlation coefficient was computed between the two metrics, which have a correlation coefficient of $\rho=.2227$. This shows that there is little correlation between the two. The two can be used to determine different physical phenomena. $\mathrm{R}^{2}$ is more useful if the phase is closer to ideal and is easier to understand and measure. Д can be used to check how periodic a signal is, which may be less useful for a detonation measurement.

Using thresholds determined from analysis, the percent of good frequencies in each configuration were determined, shown in Table 4.4. The $Д$ for Launchers 1 and 2 without wax is not shown since zero crossing analysis was not performed on these.

Table 4.4: Comparing the o of frequencies with metrics above the threshold in each analysis technique.

\begin{tabular}{|c|c|c|}
\hline Configuration & $\mathrm{R}^{2}>.995$ (\%) & Д > .85 (\%) \\
\hline Launcher 1, No Wax & 04.79 & - \\
\hline Launcher 2, No Wax & 05.76 & 02.49 \\
\hline Launcher 1, Wax & 02.43 & 18.13 \\
\hline Launcher 2, Wax & 12.76 & - \\
\hline
\end{tabular}

Table 4.5 shows the apparent frequency banding shown in the $R^{2}$ results. These are not the only bands with good frequencies, and the $\mathrm{R}^{2}$ value still varies significantly within the bands, but they are generally higher and I would recommend looking at frequencies within these bands in an experimental set-up using the RFMS.

Table 4.5: This is a selection of the wider bands that have $\mathrm{R}^{2}$ $>0.90$ which should be referenced before choosing a particular test frequency for highest odds of success in an experiment with that frequency. Shown are band start and stop frequencies and band widths.

\begin{tabular}{|l|l|l|l|}
\hline Test ID 19 & Test ID 21 & Test ID 25 & Test ID 32 \\
\hline $28.6-29.0$ & $26.5-26.8$ & $17.8-18.0$ & $20.3-20.6$ \\
$0.4 \mathrm{GHz}$ & $0.3 \mathrm{GHz}$ & $0.2 \mathrm{GHz}$ & $0.3 \mathrm{GHz}$ \\
\hline $30.4-31.3$ & $30.9-31.1$ & $20.1-20.6$ & $22.1-22.7$ \\
$0.9 \mathrm{GHz}$ & $0.2 \mathrm{GHz}$ & $0.5 \mathrm{GHz}$ & $0.6 \mathrm{GHz}$ \\
\hline $33.7-34.3$ & $32.1-32.5$ & $22.4-23.0$ & $24.5-25.9$ \\
$0.6 \mathrm{GHz}$ & $0.4 \mathrm{GHz}$ & $0.6 \mathrm{GHz}$ & $1.4 \mathrm{GHz}$ \\
\hline $36.7-38.3$ & $36.6-37.3$ & $25.2-26.2$ & $27.0-28.6$ \\
$0.6 \mathrm{GHz}$ & $0.7 \mathrm{GHz}$ & $1.0 \mathrm{GHz}$ & $1.6 \mathrm{GHz}$ \\
\hline $38.8-39.4$ & $39.5-39.8$ & $33.2-33.4$ & $32.7-34.3$ \\
$0.6 \mathrm{GHz}$ & $0.3 \mathrm{GHz}$ & $0.2 \mathrm{GHz}$ & $1.6 \mathrm{GHz}$ \\
\hline
\end{tabular}


$R^{2}$ is checked for frequency banding because $R^{2}$ is how ideal the phase unwrapping is. If a frequency outside of these bands is necessary to use, it may be better to look at Д to see if the frequency may have a repetitive phase.

\section{Conclusions}

\subsection{Summary}

Results from the measurements performed on the RFMS show that the phase behavior of the fixture is difficult to predict. The $\mathrm{R}^{2}$ metric over the frequency range used varied widely. Adding wax had an overall negative effect on launcher performance: increased S11 response. Launcher angle variation relative to the Brewster angle had no effect on system performance.

The $\mathrm{R}^{2}$ metric could be used for determining a frequency for use in an experiment using the RFMS since there were some frequencies that yielded nearly-linear phase outputs. For this particular geometry, the highest banding of $\mathrm{R}^{2}$ values occurs in Launcher 2, filled with wax, between $27 \mathrm{GHz}$ and $28.6 \mathrm{GHz}$ and between $32.7 \mathrm{GHz}$ and $34.3 \mathrm{GHz}$.

The complicated nature of the mode structure within this particular geometry is highly frequency dependent and any selected frequency may give unpredictable results if the experimental model does not match the physical test model. Because of this, there may be possible alternatives to phase-based TRA determination.

Phase itself is not reliable to determine position for this more complicated geometry, unless additional testing is performed to determine if a frequency is good to use. Model-based phase through HFSS or similar programs could be used to determine a good frequency based on what the model says should give a linear phase output. Modeling the RFMS is still a developing project. If a structure is built to accurately represent the structure to be used in an experiment, phase measurements could be taken at the frequency to be used in the experiment. Using this measurement as a calibration step, the phase yielded from the experiment could then be scaled to match the phase from calibration to determine the correct velocity.

Wavelet analysis can be used and developed, which may be less sensitive to the same kinds of errors that quadrature analysis is susceptible to, including the dependency on phase unwrapping. This 
may allow wavelet analysis to perform better at frequencies with a poor $\mathrm{R}^{2}$ metric. Wavelet analysis was not used for this experiment because it is still in development.

\subsection{Future work}

Future work on the RFMS would be to do a redesign of the fixture to allow for easier cylinder and case alignment to prevent damage to the mechanism and ensure more accurate results as well as accurate TRA alignment, which may have been off slightly. An actual detonation experiment should be used with this structure or a similar circular structure with a suitable frequency from testing to view the results and to see if detonation velocity can be extracted from the measurement. Wavelet analysis should be developed and performed on cylindrical tube and circular geometries to reduce subjective analysis decisions. Other future work includes moving the MI problem into two and three dimensional spaces, which may then be used to create images of explosion development in these experiments. LLNL may continue to use this project for research. 


\section{References}

[1] LLNL. (01DEC). History. Available: https://www.llnl.gov/about/history

[2] M. A. Cook, R. L. Doran, and G. J. Morris, "Measurement of Detonation Velocity by Doppler Effect at Three-Centimeter Wavelength," Journal of Applied Physics, vol. 26, no. 4, p. $426,1955$.

[3] V. M. Bel'skii, A. L. Mikhailov, A. V. Rodionov, and A. A. Sedov, "Microwave diagnostics of shock-wave and detonation processes," Combustion, Explosion, and Shock Waves, vol. 47, no. 6, pp. 639-650, 2011.

[4] J. W. Tringe, R. J. Kane, K. T. Lorenz, E. V. Baluyot, and K. S. Vandersall, "Dielectric characterization and microwave interferometry in HMX-based explosives," Journal of Physics: Conference Series, vol. 500, no. 14, p. 142033, Jul. 2014.

[5] J. W. Tringe, R. J. Kane, K. S. Vandersall, M. C. Converse, F. Garcia, and C. M. Tarver, "Microwave Interferometry for Understanding Deflagration-to-Detonation and Shock-toDetonation Transitions in Porous Explosives," presented at the Detonation Symposium, San Francisco, CA, United States, Jun, 2014.

[6] P. Souers, L. Lauderbach, R. Garza, L. Ferranti and P. Vitello, "Upgraded Analytical Model of the Cylinder

Test", Propellants, Explosives, Pyrotechnics, vol. 38, no. 3, pp. 419-424, 2013.

[7] (1990). A Review of Manganin Gauge Technology for Measurements in the GigaPascal Range. Available: www.dtic.mil/cgi-bin/GetTRDoc?AD=ADA227927

[8] NASA. (1974). TM X-72625, Interferometry Technique for Obtaining Gas Interface Velocity Measurements in an Expansion Tube Facility (NASA).

[9] G. H. McCall, W. L. Bongianni, and G. A. Miranda, "Microwave interferometer for shock wave, detonation, and material motion measurements," Review of Scientific Instruments, vol. 56, no. 8, p. 1612, 1985.

[10] (1965). S-87, A Microwave Technique for Studying Detonation Phenomena.

[11] B. C. Glancy, H. W. Sandusky, and A. D. Krall, "Microwave interferometry of shock waves. II. Reacting porous media," Journal of Applied Physics, vol. 74, no. 10, p. 6328, 1993.

[12] A. D. Krall, B. C. Glancy, and H. W. Sandusky, "Microwave interferometry of shock waves. I. Unreacting porous media," Journal of Applied Physics, vol. 74, no. 10, p. 6322, 1993.

[13] J. J. Lee, G. Dupre, R. Knystautas, and J. H. Lee, "Doppler Interferometry Study of Unstable Detonations," pp. 175-181, 1995.

[14] J. Choi et al., "Microwave Interferometer for Shock Wave Induced Displacement Measurement," pp. 1722-1726, 2011. 
[15] F. L. Tevelow, "Microwave Interferometer Measurements in Shocked Air," (in English), Journal of Applied Physics, vol. 38, no. 4, pp. 1765-1780, 1967.

[16] P. Rae, B. Glover, J. Gunderson, and L. Perry, "Free-field microwave interferometry for detonation front tracking and run-to-detonation measurements," pp. 434-437, 2012.

[17] R. J. Kane, "Microwave Interferometry Data Analysis," 2015.

[18] R. J. Kane, J. W. Tringe, G. L. Klunder, E. V. Baluyot, J. M. Densmore, and M. C. Converse, "Microwave Interrogation of An Air Plasma Plume as a Model system for Hot Spots in Explosives."

[19] L. Wang, R. Simpkin, and A. M. Al-Jumaily, "3D breast cancer imaging using holographic microwave interferometry," in Conference on Image and Vision Computing, Dunedid, New Zealand, 2012, pp. 180-185.

[20] S. Gonzales, "MI Test Apparatus," 2016.

[21] C. Balanis, Advanced Engineering Electromagnetics. Hoboken, NJ: John Wiley \& Sons, Inc., 1989.

[22] D. Pozar, Microwave Engineering, 4th ed. Hoboken, NJ: John Wiley \& Sons, Inc., 2012.

[23] "DAK $5 \mathrm{GHz}$ - 50 (67) GHz," SPEAG. [Online]. Available: https://www.speag.com/products/dak/dak-dielectric-probesystems/dak-5-50-67-ghz/. [Accessed: 02-Jun-2017]. 


\section{APPENDICES}

Appendix A. Auspices and Disclaimers

Auspices and disclaimer statements

This work was performed under the auspices of the U.S. Department of Energy by Lawrence Livermore National Laboratory in part under Contract W-7405-Eng-48 and in part under Contract DE-AC52$07 N A 27344$.

This document was prepared as an account of work sponsored by an agency of the United States government. Neither the United States government nor Lawrence Livermore National Security, LLC, nor any of their employees makes any warranty, expressed or implied, or assumes any legal liability or responsibility for the accuracy, completeness, or usefulness of any information, apparatus, product, or process disclosed, or represents that its use would not infringe privately owned rights. Reference herein to any specific commercial product, process, or service by trade name, trademark, manufacturer, or otherwise does not necessarily constitute or imply its endorsement, recommendation, or favoring by the United states government or Lawrence Livermore National Security, LLC. The views and opinions of authors expressed herein do not necessarily state or reflect those of the United States government or Lawrence Livermore National Security, LLC, and shall not be used for advertising or product endorsement purposes.

LLNL-TH-733023-DRAFT 


\section{Appendix B. Phase and Magnitude Sample Results for Different Launcher Configurations}

This appendix is meant to show sample results from the RFMS. It is neither comprehensive not meant to show specific usable data.

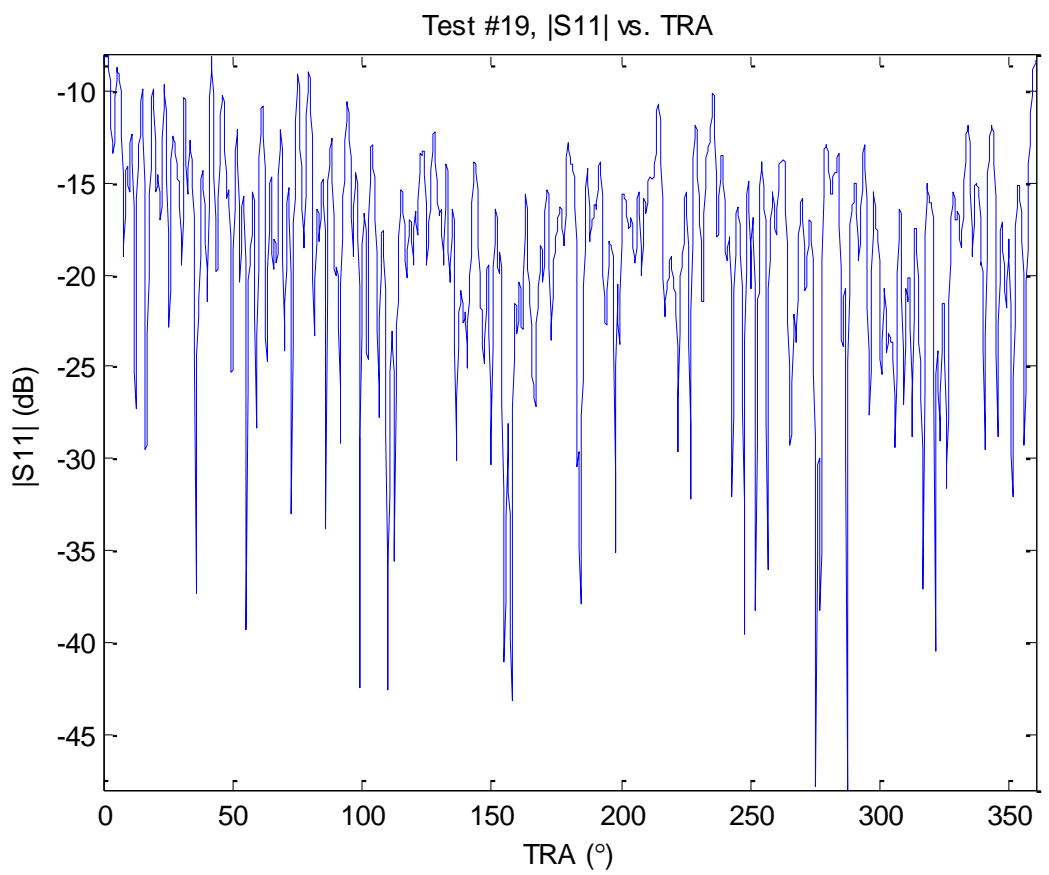

Figure A.1: Magnitude of S11 vs. TRA at 39.1978GHz for Test \#19.

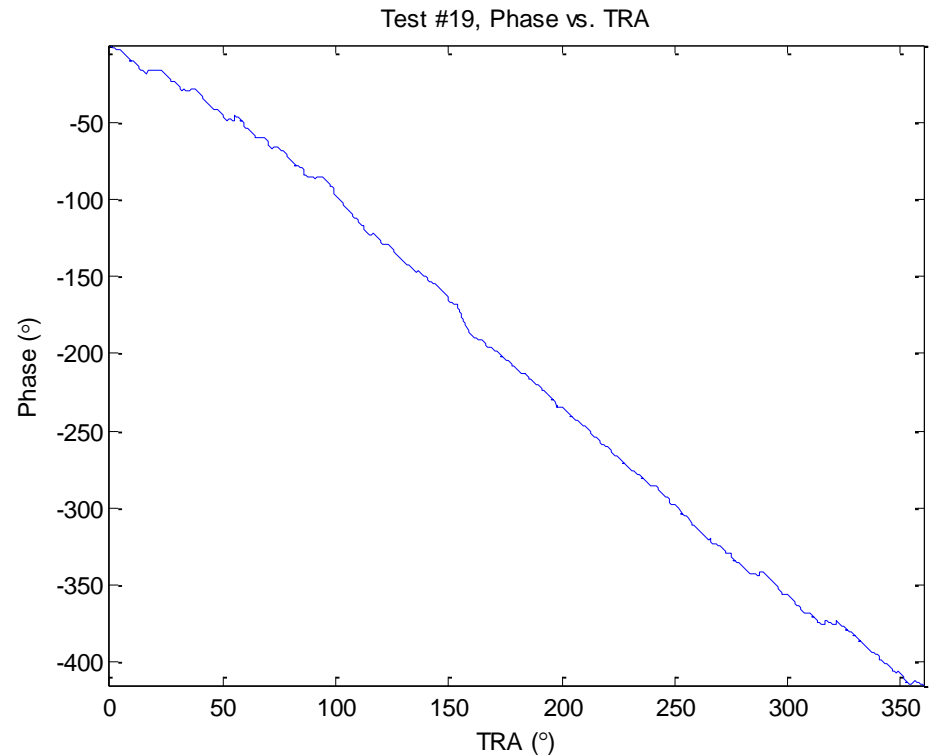

Figure A.2: S11 Phase vs. TRA at 39.1978GHz for Test \#19. 


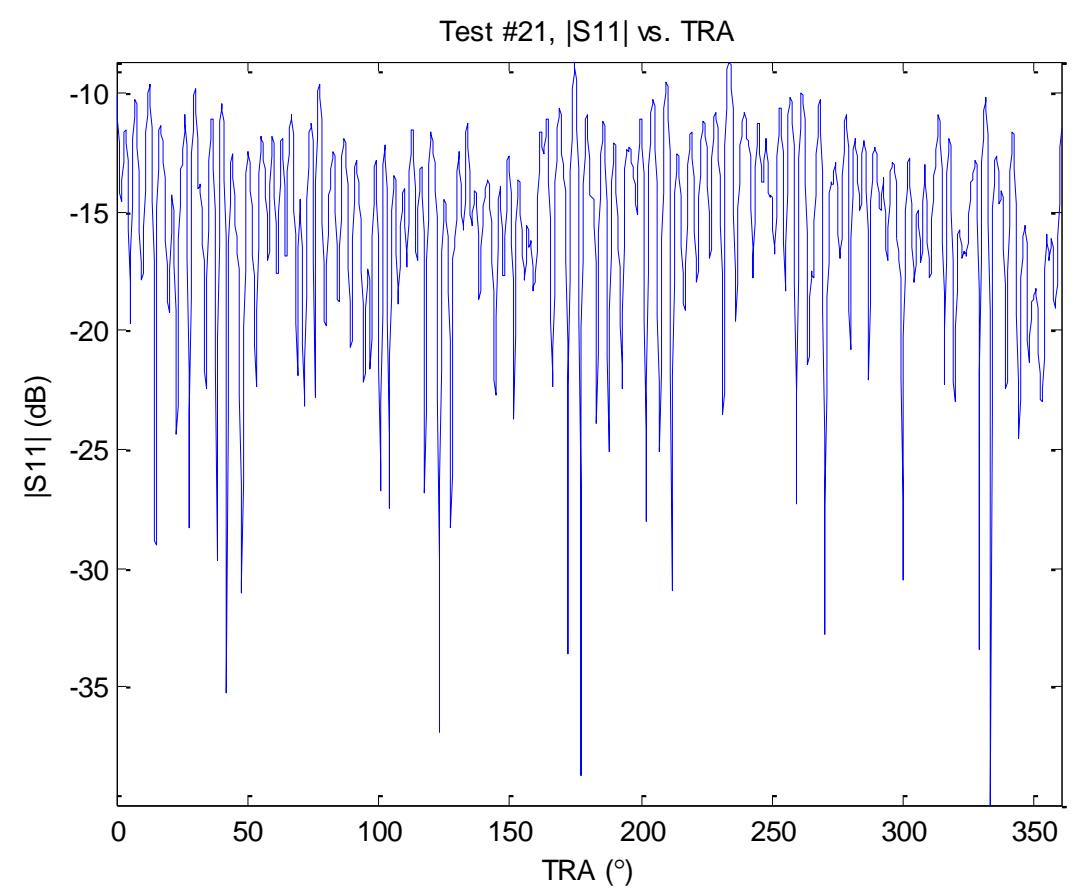

Figure A.3: Magnitude of S11 vs. TRA at 39.1978GHz for Test \#21.

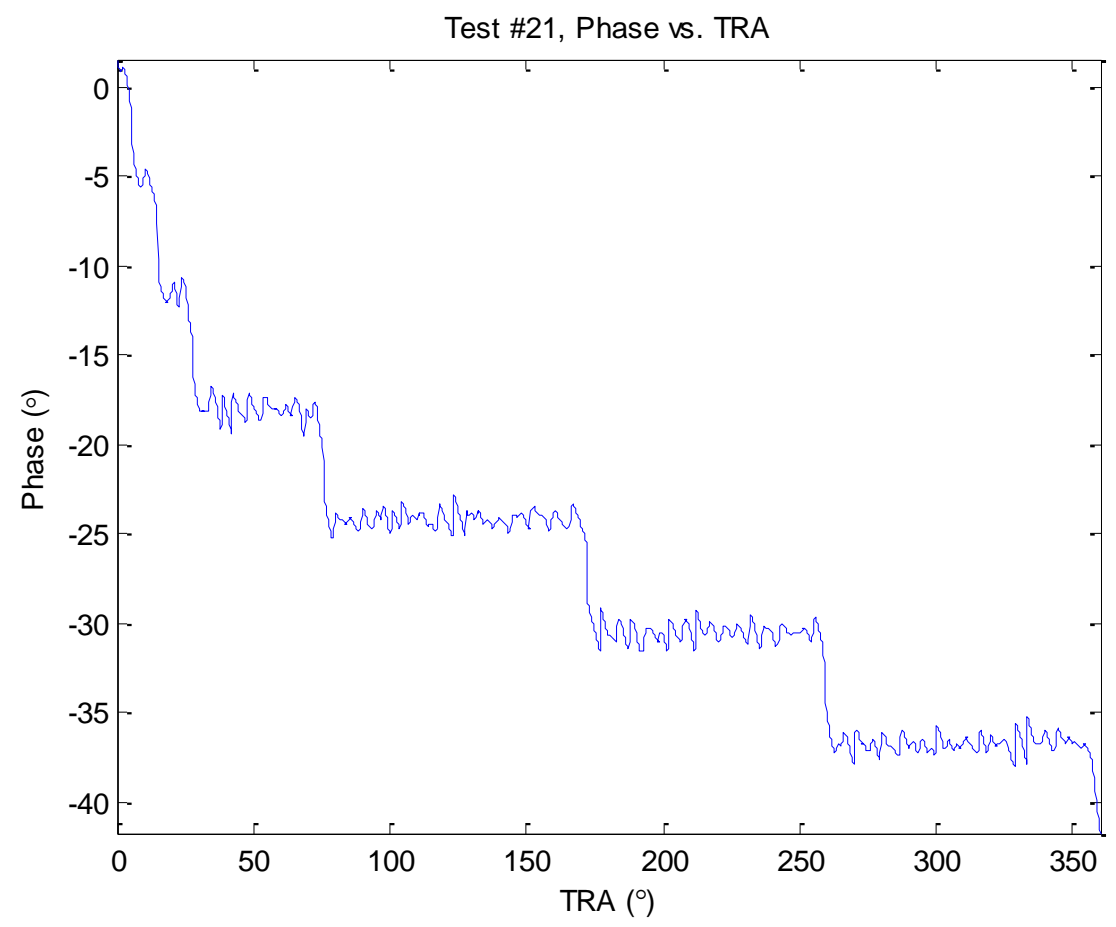

Figure A.4: S11 Phase Vs. TRA at 39.1978GHz for Test \#21. 
Test \#25, |S11| vs. TRA

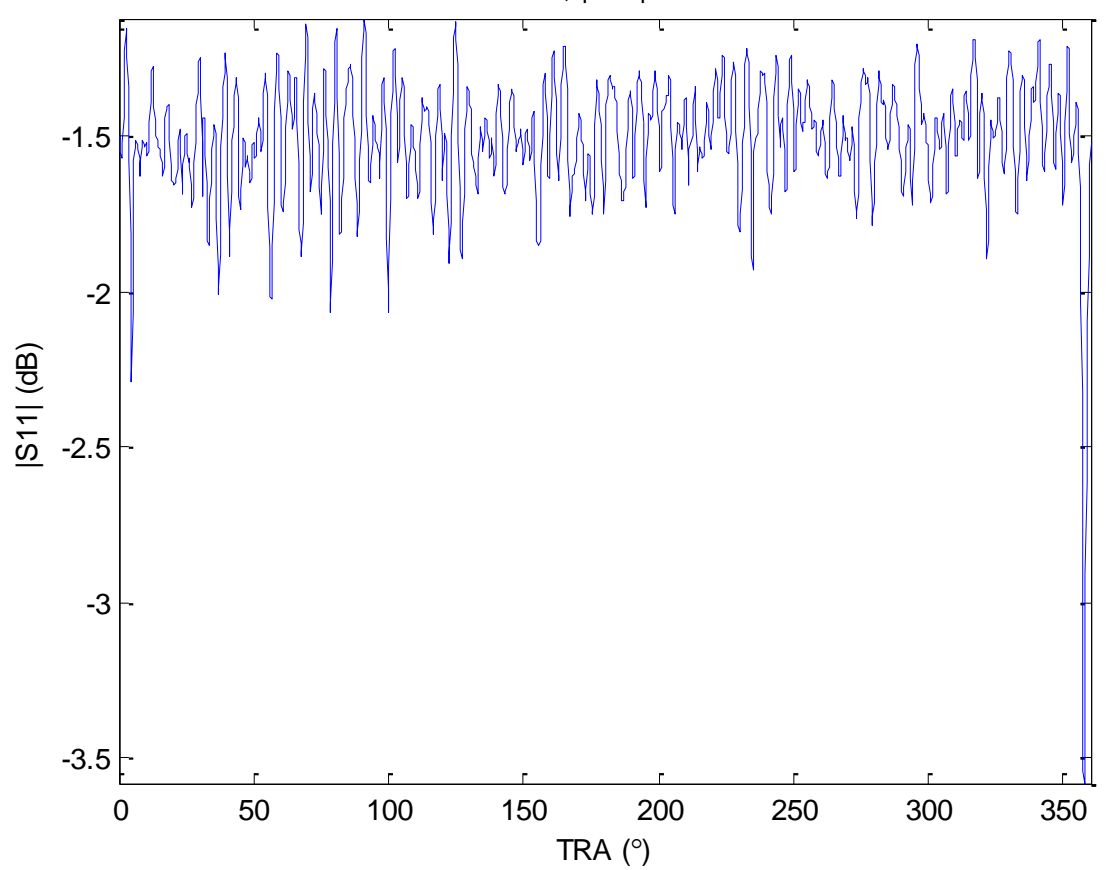

Figure A.5: Magnitude of S11 vs. TRA at 39.1977GHz for Test \#25.

Test \#25, Phase vs. TRA

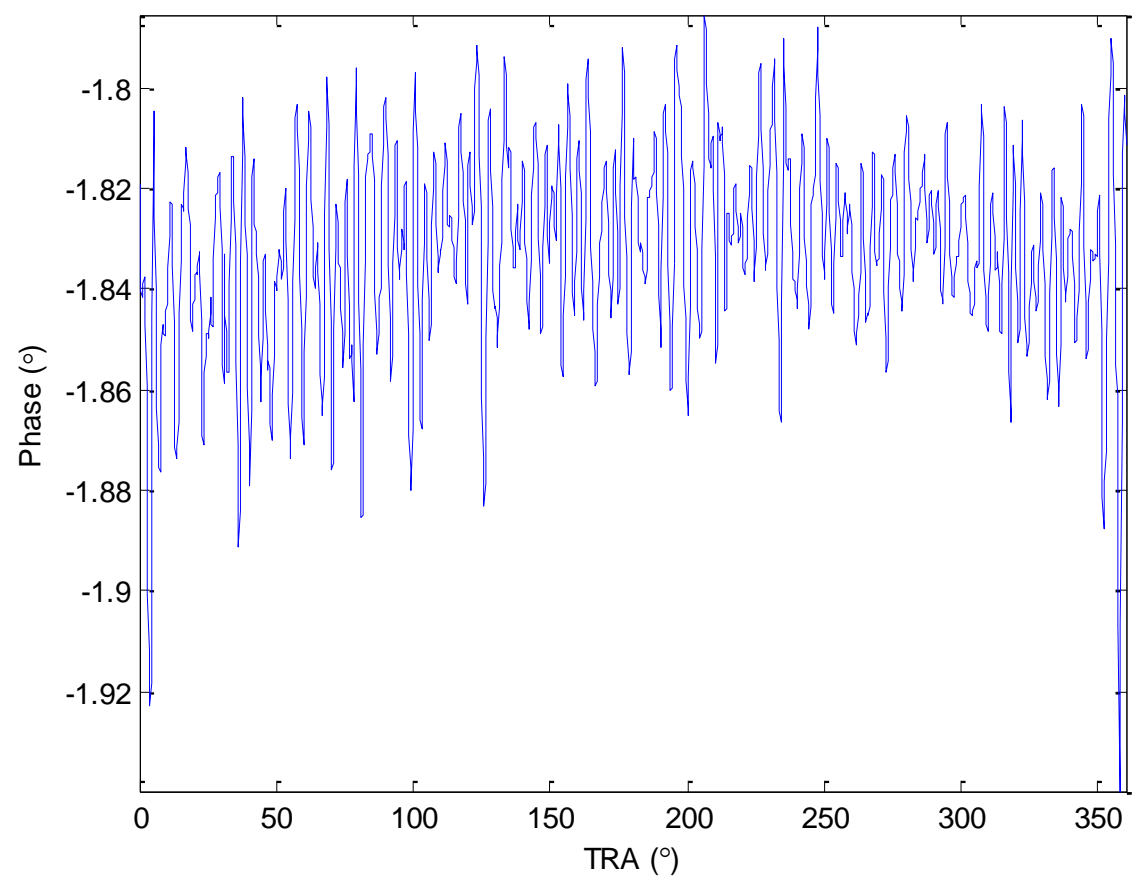

Figure A.6: S11 Phase vs. TRA at 39.1977GHz for Test \#25. 


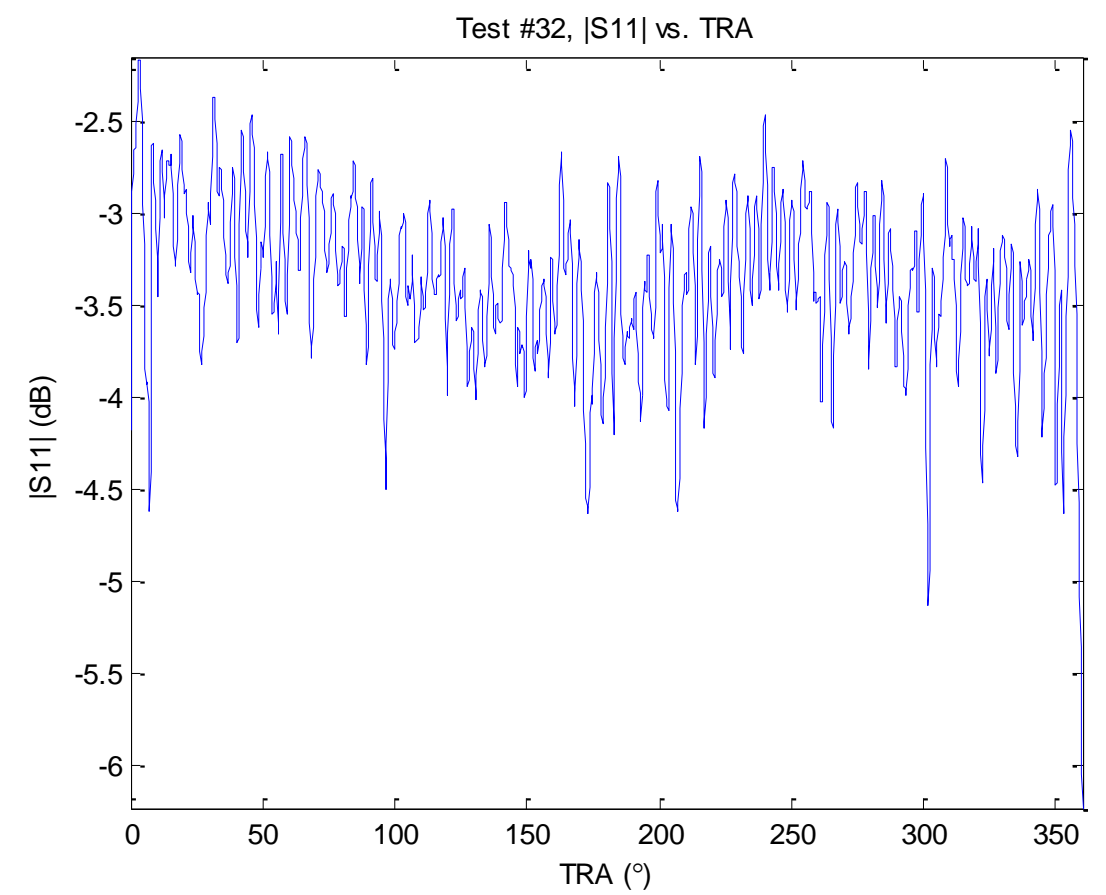

Figure A.7: Magnitude of S11 vs. TRA at 39.1977GHz for Test \#32.

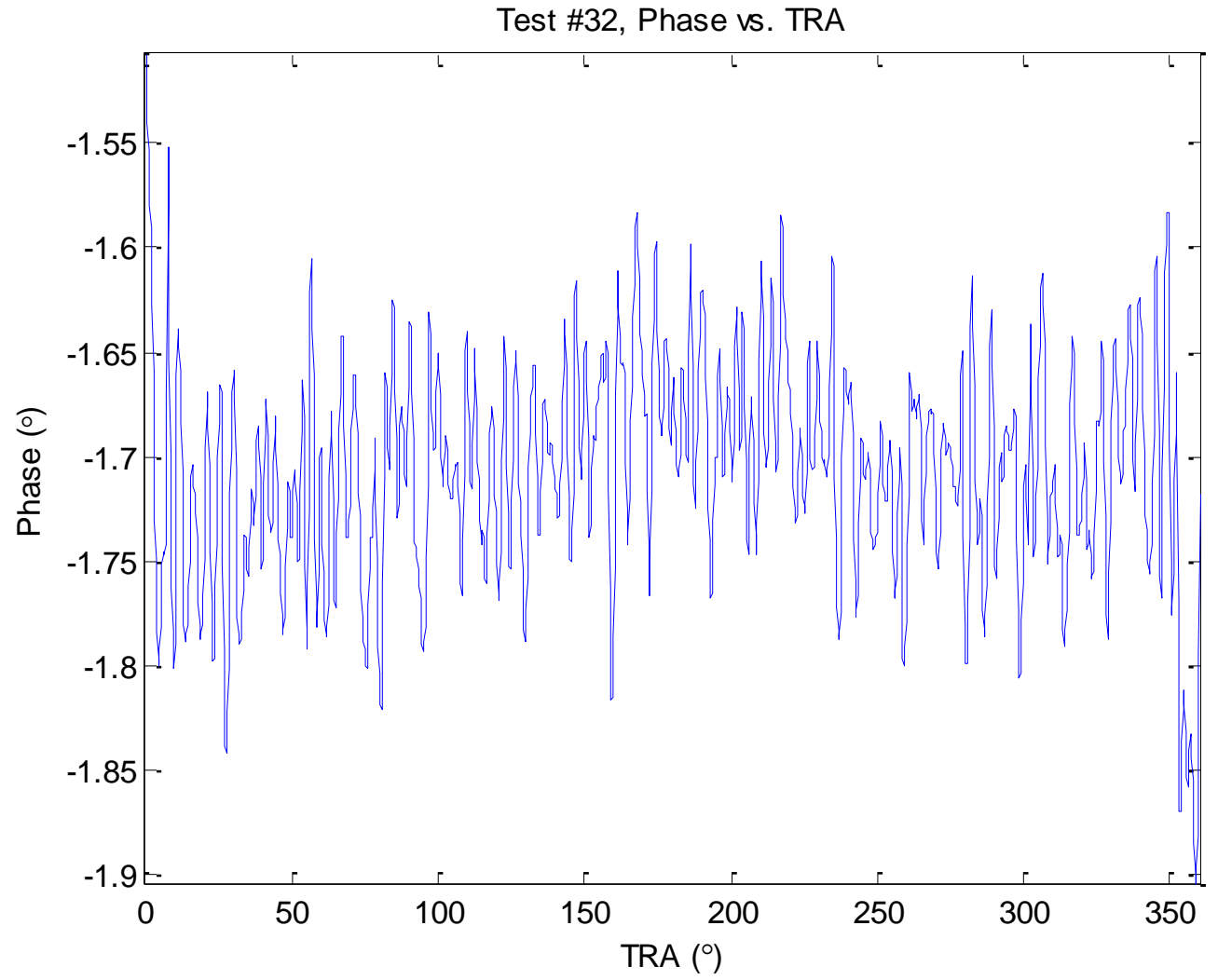

Figure A.8: S11 Phase vs. TRA at 39.1977GHz for Test \#32. 


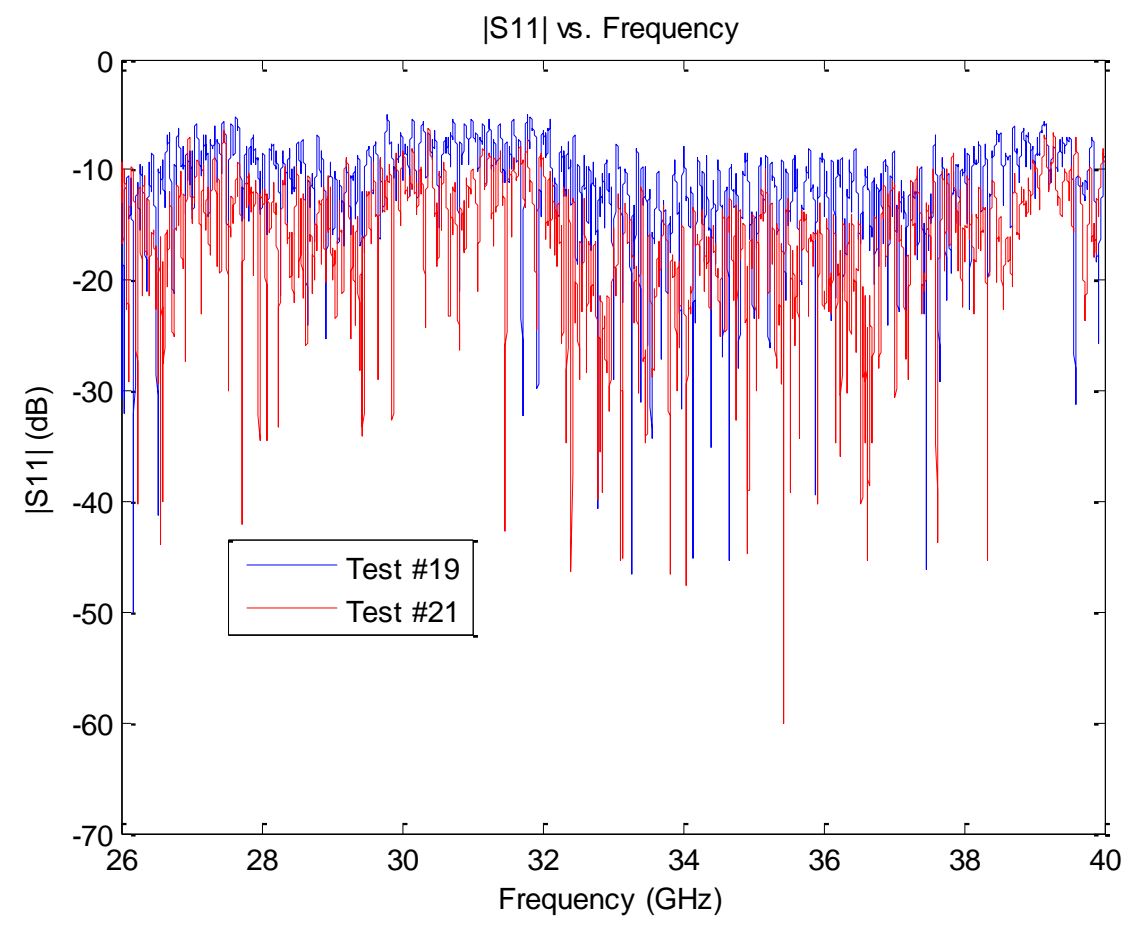

Figure A.9: Magnitude of S11 vs. Frequency at $0^{\circ}$ TRA for Tests $\# 19$ and \#21.

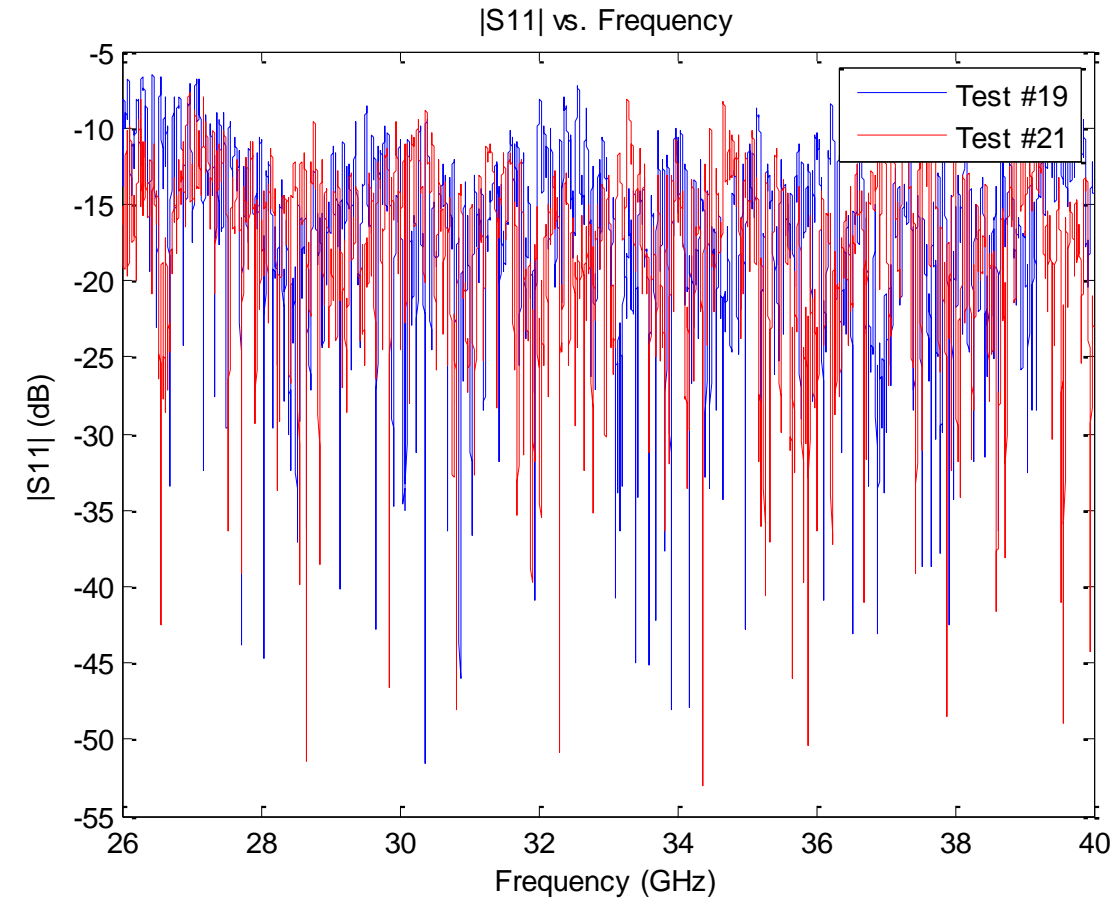

Figure A.10: Magnitude of S11 vs. Frequency at $45^{\circ}$ TRA for Tests \#19 and \#21. 


\section{Appendix C. $R^{2}$ and $Д$ Results for Different Launcher Configurations}

This appendix is meant to show sample results from the RFMS. It is neither comprehensive not meant to show specific usable data.

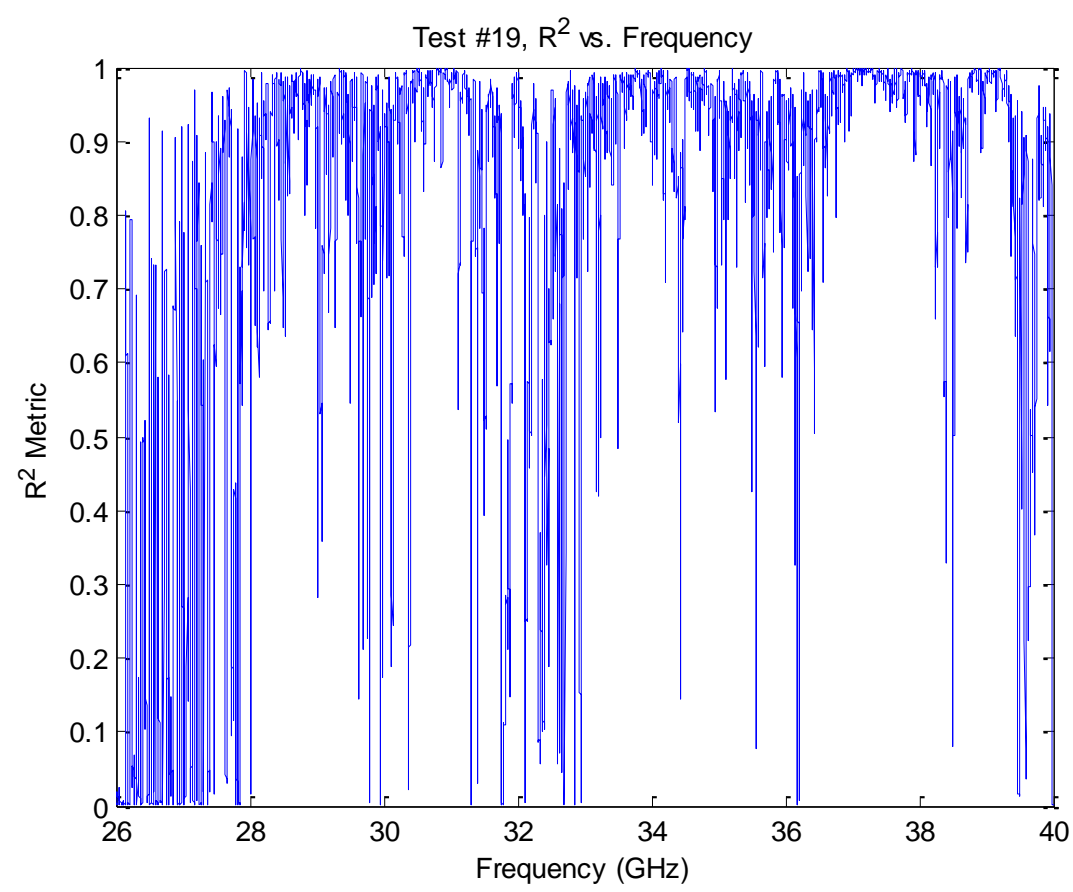

Figure A.11: R² VS. Frequency for Test \#19. 


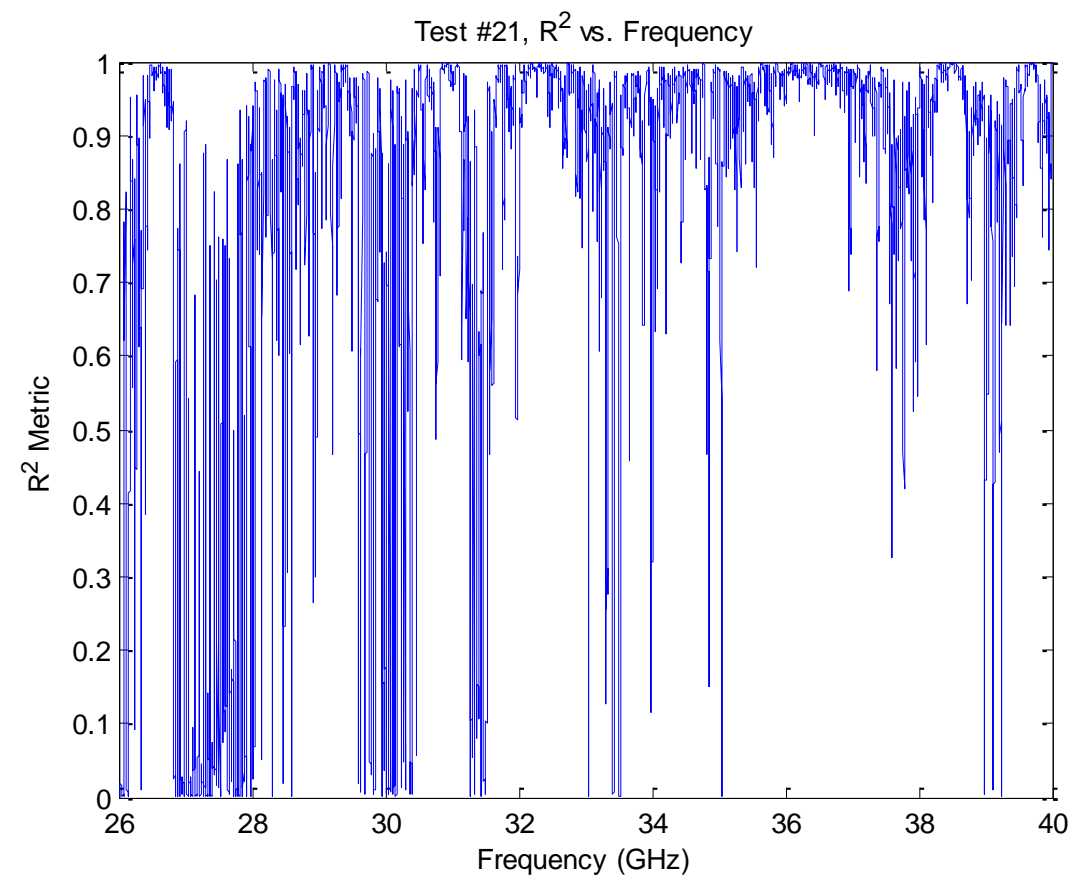

Figure A.12: R ${ }^{2}$ Vs. Frequency for Test \#21.

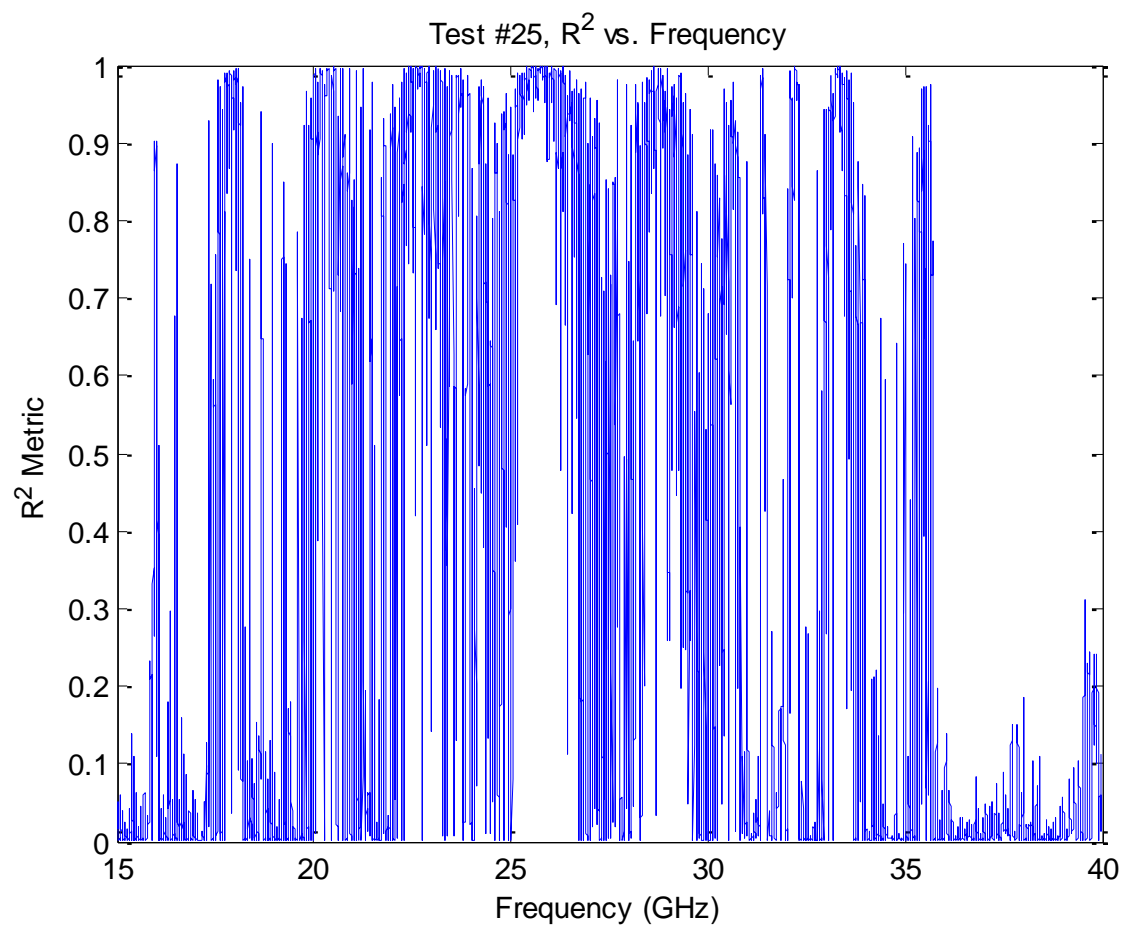

Figure A.13: $R^{2}$ Vs. Frequency for Test \#25. 


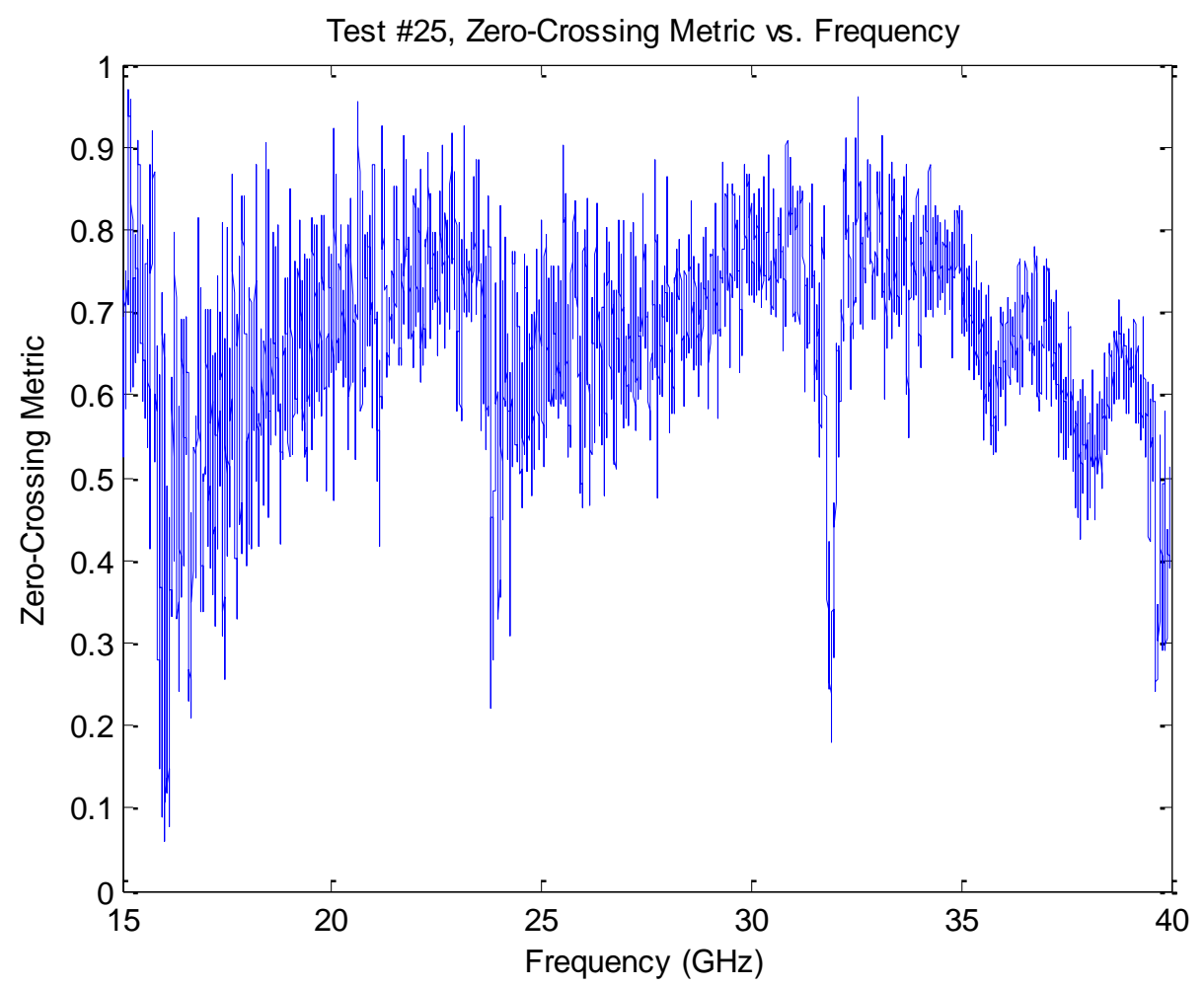

Figure A.14: Д vs. Frequency for Test \#25.

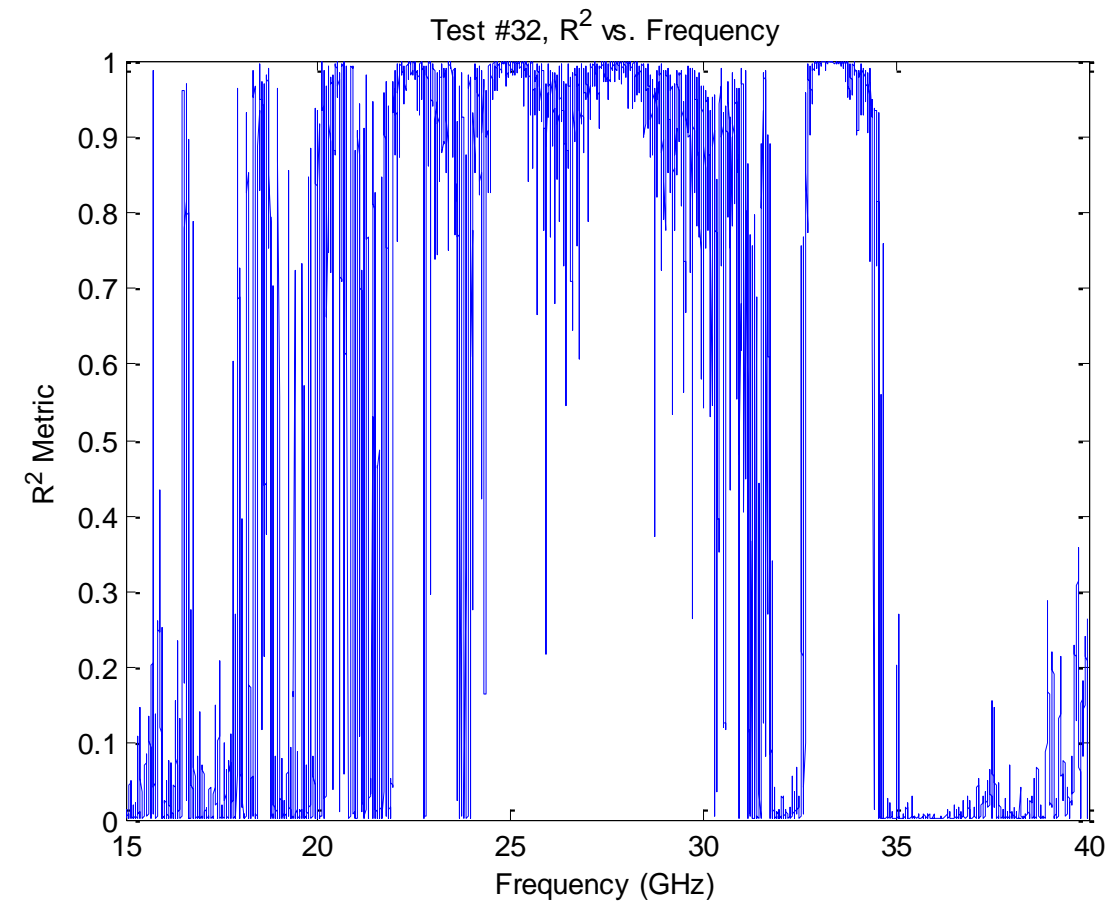

Figure A.15: $R^{2}$ Vs. Frequency for Test \#32. 


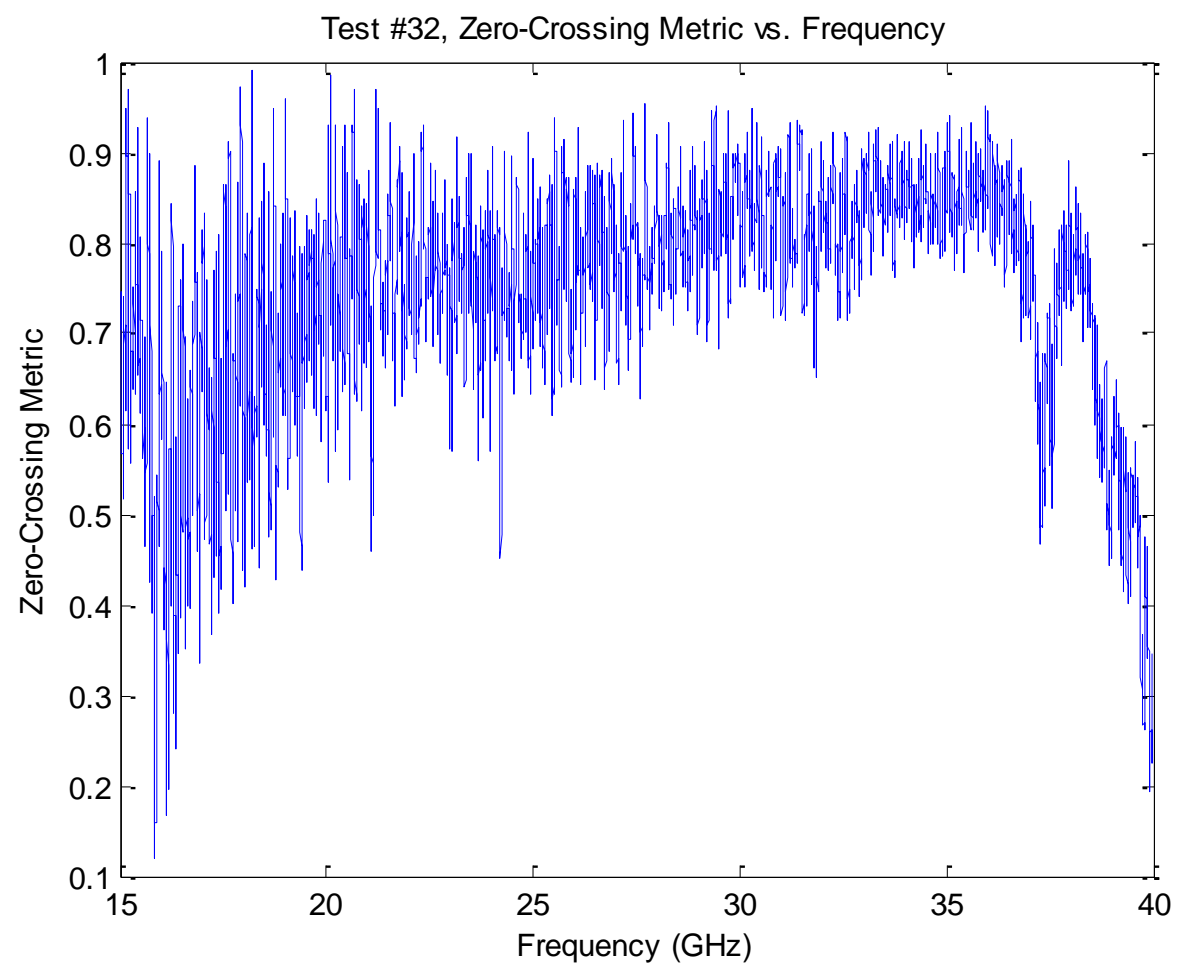

Figure А.16: Д VS. Frequency for Test \#32. 